TRANSACTIONS OF THE

AMERICAN MATHEMATICAL SOCIETY

Volume 361, Number 6, June 2009, Pages 2871-2911

S 0002-9947(09)04924-1

Article electronically published on January 22, 2009

\title{
ENTIRE FUNCTIONS MAPPING UNCOUNTABLE DENSE SETS OF REALS ONTO EACH OTHER MONOTONICALLY
}

\author{
MAXIM R. BURKE
}

\begin{abstract}
When $A$ and $B$ are countable dense subsets of $\mathbb{R}$, it is a well-known result of Cantor that $A$ and $B$ are order-isomorphic. A theorem of K.F. Barth and W.J. Schneider states that the order-isomorphism can be taken to be very smooth, in fact the restriction to $\mathbb{R}$ of an entire function. J.E. Baumgartner showed that consistently $2^{\aleph_{0}}>\aleph_{1}$ and any two subsets of $\mathbb{R}$ having $\aleph_{1}$ points in every interval are order-isomorphic. However, U. Abraham, M. Rubin and S. Shelah produced a ZFC example of two such sets for which the orderisomorphism cannot be taken to be smooth. A useful variant of Baumgartner's result for second category sets was established by S. Shelah. He showed that it is consistent that $2^{\aleph_{0}}>\aleph_{1}$ and second category sets of cardinality $\aleph_{1}$ exist while any two sets of cardinality $\aleph_{1}$ which have second category intersection with every interval are order-isomorphic. In this paper, we show that the orderisomorphism in Shelah's theorem can be taken to be the restriction to $\mathbb{R}$ of an entire function. Moreover, using an approximation theorem of L. Hoischen, we show that given a nonnegative integer $n$, a nondecreasing surjection $g: \mathbb{R} \rightarrow \mathbb{R}$ of class $C^{n}$ and a positive continuous function $\epsilon: \mathbb{R} \rightarrow \mathbb{R}$, we may choose the order-isomorphism $f$ so that for all $i=0,1, \ldots, n$ and for all $x \in \mathbb{R}$, $\left|D^{i} f(x)-D^{i} g(x)\right|<\epsilon(x)$.
\end{abstract}

\section{INTRODUCTION}

When $A$ and $B$ are countable dense subsets of $\mathbb{R}$, it is a well-known result of Cantor [Ca, $\S 9]$ that $A$ and $B$ are order-isomorphic. Notice that an orderisomorphism between dense subsets of $\mathbb{R}$ extends to an order-isomorphism of $\mathbb{R}$. We record this simple fact as a proposition for ease of reference.

Proposition 1.1. If $K, L \subseteq \mathbb{R}$ are dense and $h: K \rightarrow L$ is an order isomorphism, then $h$ extends to an order isomorphism of $\mathbb{R}$.

The extension to an order-isomorphism of $\mathbb{R}$ of an isomorphism between countable dense sets given by Cantor's theorem is in particular a monotone function and hence differentiable almost everywhere. The question of improving the isomorphism was examined by Franklin $[\mathrm{Fr}$, who showed that it can be taken to be real-analytic. Motivated by the problem of finding order-isomorphisms of $[0,1]$ which map each of

Received by the editors March 10, 2006.

2000 Mathematics Subject Classification. Primary 03E35; Secondary 30E10.

Key words and phrases. Order-isomorphism, second category, entire function, oracle-cc forcing, Carleman's theorem, Hoischen's theorem.

The author's research was supported by NSERC. The author thanks F.D. Tall and the Department of Mathematics at the University of Toronto for their hospitality during the academic year 2003/2004 when much of the present paper was written.

(C)2009 American Mathematical Society 
the sets of rational, algebraic and transcendental numbers onto themselves, Melzak Me observes that Franklin's methods show that if $\left\{A_{n}\right\}_{n<\omega}$ and $\left\{B_{n}\right\}_{n<\omega}$ are each a sequence of pairwise disjoint countable dense subsets of $(0,1)$, then there is an analytic order-isomorphism $f$ of $[0,1]$ such that for each $n<\omega$ the function $f$ maps $A_{n}$ onto $B_{n}$. Moreover, given any function $g$ of class $C^{n}$ whose derivative is bounded away from zero, $f$ can be chosen so that its first $n$ derivatives are uniformly approximated by those of $g$. The map in Franklin's result was improved to being the restriction to $\mathbb{R}$ of an entire function by Barth and Schneider [BS], thereby solving [Er, Problem 24]. They also state without proof that their method gives the generalization to sequences of pairwise disjoint countable dense sets as obtained by Melzak for analytic functions, but that "the massive amount of bookkeeping involved in this proof is such as to make it impractical to include it in this paper". A variation on the problem of Erdös referred to above, interpreted so that it refers to countable dense subsets of $\mathbb{C}$ rather than of $\mathbb{R}$, was solved by Maurer Ma. An elegant proof of the Barth-Schneider result based on Maurer's work was given by Sato and Rankin [SR. (See also $[\mathrm{NT}$, which contains a variation on the same argument.) They make no comment about the result for sequences of pairwise disjoint countable dense sets, but their proof easily yields that version as well.

In $\left[\mathrm{Ba}\right.$, a nonempty set $S$ of real numbers is said to be $\aleph_{1}$-dense if $S$ is without endpoints and there are exactly $\aleph_{1}$ members of $S$ between any two distinct points of $S$. In particular, if $S \cap I$ has cardinality $\aleph_{1}$ for every nonempty open interval $I$, then $S$ is $\aleph_{1}$-dense. We shall use the term only in this more restricted sense. Baumgartner proved [Ba] that if ZFC is consistent, then so is the theory ZFC + the statement "all $\aleph_{1}$-dense sets of reals are order-isomorphic", which is a natural stepping-up by one cardinal of Cantor's theorem. Following Abraham, Rubin and Shelah ARS, we denote the statement in quotes by BA. (The two meanings of $\aleph_{1}$-dense are equivalent for the purposes of BA.) It is shown in ARS that the function inducing the order-isomorphisms in BA cannot in general be taken to be smooth. We reproduce the argument from [ARS, giving a few more details. Our statement of the result is slightly different; see Remark 1.4.

Proposition 1.2 ([ARS, Proposition 9.4]). There are $\aleph_{1}$-dense sets $A, B \subseteq \mathbb{R}$ such that for no nonconstant $C^{1}$ function $f: \mathbb{R} \rightarrow \mathbb{R}$ do we have $f[A] \subseteq B$.

Proof. Fix a one-to-one enumeration $\left\{r_{n}: n<\omega\right\}$ of $\mathbb{Q}$. We define strictly increasing functions $g, h: \mathbb{Q} \rightarrow \mathbb{R}$ and, letting $a_{n}=g\left(r_{n}\right)$ and $b_{n}=h\left(r_{n}\right)$, satisfy

$$
\min \left\{\left|a_{i}-a_{j}\right|,\left|b_{k}-b_{\ell}\right|\right\}<\max \left\{\left|a_{i}-a_{j}\right|,\left|b_{k}-b_{\ell}\right|\right\}^{2}
$$

whenever $i \neq j$ and $k \neq \ell$. The definition proceeds by inductively choosing $a_{0}, b_{0}, a_{1}, b_{1}, \ldots$. Choose $a_{0}$ and $b_{0}$ arbitrarily. If $n \geq 1$ and $a_{i}, b_{i}$ have been chosen for $i<n$ and (1) holds whenever the indices are smaller than $n$, pick $a_{n}$ and $b_{n}$ as follows. Choose an index $i_{0}<n$ such that $r_{i_{0}}$ is adjacent to $r_{n}$ in $\left\{r_{i}: i \leq n\right\}$. The choice of $a_{n}$ must be made so that the instances of (1.1) of the form

$$
\min \left\{\left|a_{i}-a_{n}\right|,\left|b_{k}-b_{\ell}\right|\right\}<\max \left\{\left|a_{i}-a_{n}\right|,\left|b_{k}-b_{\ell}\right|\right\}^{2},
$$

where $i<n$ and $k, \ell<n$ are distinct, hold. When $i \neq i_{0}$, the fact that

$$
\min \left\{\left|a_{i}-a_{i_{0}}\right|,\left|b_{k}-b_{\ell}\right|\right\}<\max \left\{\left|a_{i}-a_{i_{0}}\right|,\left|b_{k}-b_{\ell}\right|\right\}^{2}
$$

ensures that as long as $a_{n}$ is close enough to $a_{i_{0}}$, (1.2) will hold. When $i=i_{0}$, as long as $a_{n}$ is close enough to $a_{i_{0}}$, we have $\left|a_{i_{0}}-a_{n}\right|<\left|b_{k}-b_{\ell}\right|$, and moreover 
$\left|a_{i_{0}}-a_{n}\right|<\left|b_{k}-b_{\ell}\right|^{2}$ whenever $k, \ell<n$ are distinct. Thus choosing $a_{n}$ close enough to $a_{i_{0}}$ gives (1.1) for all distinct indices $i, j \leq n$ and all distinct indices $k, \ell<n$. Hence we choose $a_{n}$ so that it is close enough to $a_{i_{0}}$, as just described, and $g\left\lceil\left\{r_{i}: i \leq n\right\}\right.$ is order-preserving. (When $n=1$, the first part is vacuous since there do not exist distinct $k, \ell<n$.) The choice of $b_{n}$ is completely analogous. Now let $L=\operatorname{cl}(g[\mathbb{Q}]), M=\operatorname{cl}(h[\mathbb{Q}])$. (cl denotes closure in $\mathbb{R}$.) The points of $L$ can be approximated arbitrarily well by points $a_{n}$ and those of $M$ by points $b_{n}$. It follows that for any points $a, a^{\prime} \in L$ and $b, b^{\prime} \in M$ we have

$$
\min \left\{\left|a-a^{\prime}\right|,\left|b-b^{\prime}\right|\right\} \leq \max \left\{\left|a-a^{\prime}\right|,\left|b-b^{\prime}\right|\right\}^{2} .
$$

(Notice that (1.3) is trivially true when $a=a^{\prime}$ or $b=b^{\prime}$. Use (1.1) for the case $a \neq a^{\prime}$ and $b \neq b^{\prime}$.) Since the sets $g[\mathbb{Q}]$ and $h[\mathbb{Q}]$ have the order type of the rationals, their closures are uncountable. (In each set, distinct initial segments have distinct suprema.) Choose sets of cardinality $\aleph_{1}, A_{L} \subseteq L$ and $B_{M} \subseteq M$. Let $A=A_{L}+\mathbb{Q}$, $B=B_{M}+\mathbb{Q}$.

Consider a continuous function $f: \mathbb{R} \rightarrow \mathbb{R}$ such that $f[A] \subseteq B$.

Claim 1.3. In each nonempty open interval $I$, either $f$ has divided differences $|f(a)-f(b)| /|a-b|, a \neq b$, which are arbitrarily large, or $f$ has divided differences which are arbitrarily close to zero.

Proof of Claim. Fix a nonempty open interval $I$. For each $x \in A \cap I$, there are $q_{x}, r_{x} \in \mathbb{Q}$ such that $x \in A_{L}+q_{x}, f(x) \in B_{M}+r_{x}$. Since $A \cap I$ is uncountable, there are $q, r \in \mathbb{Q}$ and an uncountable set $S \subseteq A \cap I$ such that for all $x \in S, q_{x}=q$ and $r_{x}=r$. Choose any accumulation point $a \in I$ for the set $S$, and pick a sequence of distinct points $\left\{s_{n}\right\} \subseteq S$ converging to $a$. Then $\left\{f\left(s_{n}\right)\right\}$ converges to $f(a)$ since $f$ is continuous. Given any $\varepsilon>0$, fix a large enough $n$ so that $\left|s_{n}-s_{n+1}\right|<\varepsilon$ and $\left|f\left(s_{n}\right)-f\left(s_{n+1}\right)\right|<\varepsilon$. By (1.3),

$$
\begin{aligned}
\min \left\{\left|s_{n}-s_{n+1}\right|,\left|f\left(s_{n}\right)-f\left(s_{n+1}\right)\right|\right\} & \leq \max \left\{\left|s_{n}-s_{n+1}\right|,\left|f\left(s_{n}\right)-f\left(s_{n+1}\right)\right|\right\}^{2} \\
& \leq \varepsilon \max \left\{\left|s_{n}-s_{n+1}\right|,\left|f\left(s_{n}\right)-f\left(s_{n+1}\right)\right|\right\},
\end{aligned}
$$

which implies that the divided difference $d_{\varepsilon}=\left|f\left(s_{n}\right)-f\left(s_{n+1}\right)\right| /\left|s_{n}-s_{n+1}\right|$ is either $\leq \varepsilon$ or $\geq 1 / \varepsilon$. At least one of $d_{\varepsilon} \leq \varepsilon$ or $d_{\varepsilon} \geq 1 / \varepsilon$ must occur for values of $\varepsilon$ arbitrarily close to zero. This establishes Claim 1.3 .

The conclusion now follows from the claim since a nonconstant $C^{1}$ function will have intervals over which the values of its derivative, and hence also its divided differences, are in a bounded closed interval not containing zero.

Remark 1.4. Consider the case of the proposition where $f$ is an order-isomorphism. The point $a$ in the above argument can be taken to be in $S$ and to be a two-sided accumulation point of $S$. The sequence $\left\{s_{n}\right\}$ can then be chosen so that $s_{n}$ and $s_{n+1}$ are on different sides of $a$. Then $\left|f\left(s_{n}\right)-f\left(s_{n+1}\right)\right| /\left|s_{n}-s_{n+1}\right|>1 / \varepsilon$ gives either $\left|f\left(s_{n}\right)-f(a)\right| /\left|s_{n}-a\right|>1 / \varepsilon$ or $\left|f(a)-f\left(s_{n+1}\right)\right| /\left|a-s_{n+1}\right|>1 / \varepsilon$. Similarly in the $<\varepsilon$ situation. Hence either $D f(a)$ does not exist or $D f(a)=0$. In ARS, the stronger conclusion that for some $a \in A, D f(a)$ does not exist is stated. We do not see why this stronger conclusion holds.

Remark 1.5. Since order-isomorphisms of $\mathbb{R}$ are homeomorphisms, one consequence of Proposition 1.1 is that dense subsets of $\mathbb{R}$ which are order-isomorphic must be indistinguishable topologically as subspaces of $\mathbb{R}$. In particular, a dense first 
category set cannot be order isomorphic to a dense second category set. There is always an $\aleph_{1}$-dense first category set. (Cf. the proof of Proposition 1.2.) Hence, $\mathrm{BA}$ implies that all sets of cardinality $\aleph_{1}$ are first category.

Notice that the sets $A$ and $B$ given by the proof of Proposition 1.2 are first category. Shelah proved the following theorem as part of the proof of Sh1980, Theorem 4.7], which states that if ZFC is consistent, then so is ZFC $+2^{\aleph_{0}}=\aleph_{2}+$ "there is a universal (linear) order of power $\aleph_{1}$ ".

Theorem 1.6 ([Sh1980]). If ZFC is consistent, then so is ZFC + both of the following statements.

(a) There is a second category set in $\mathbb{R}$ of cardinality $\aleph_{1}$.

(b) Let $A$ and $B$ be everywhere second category subsets of $\mathbb{R}$ of cardinality $\aleph_{1}$. Then $A$ and $B$ are order-isomorphic.

(A set $A \subseteq \mathbb{R}$ is everywhere second category if $A \cap I$ is second category for every nonempty open interval $I$.)

An examination of Shelah's model shows that, by a simple genericity argument, the functions witnessing (b) fail to be differentiable at any constructible real. In this paper we show that the order-isomorphism in Theorem 1.6 can be taken to be the restriction to $\mathbb{R}$ of an entire function. The following is the main result of this paper.

Theorem 1.7. If ZFC is consistent, then so is $Z F C+2^{\aleph_{0}}=\aleph_{2}+$ the following statements.

(a) Every second category set in $\mathbb{R}$ has a second category subset of cardinality $\aleph_{1}$.

(b) For any two sequences, $\left\langle A_{\alpha}: \alpha<\omega_{1}\right\rangle$ and $\left\langle B_{\alpha}: \alpha<\omega_{1}\right\rangle$, each consisting of pairwise disjoint dense subsets of $\mathbb{R}$, if $A_{\alpha}$ and $B_{\alpha}$ are countable for $\alpha<\omega$ and are everywhere second category sets of cardinality $\aleph_{1}$ for $\omega \leq \alpha<\omega_{1}$, then there is an entire function $f: \mathbb{C} \rightarrow \mathbb{C}$ which restricts to an orderisomorphism of $\mathbb{R}$ such that $f\left[A_{\alpha}\right]=B_{\alpha}$ for every $\alpha<\omega_{1}$.

(c) Suppose that in (b) we are additionally given a positive continuous function $\varepsilon: \mathbb{R} \rightarrow \mathbb{R}$ and a nondecreasing surjection $g: \mathbb{R} \rightarrow \mathbb{R}$.

(i) If $n$ is a nonnegative integer and $g$ is of class $C^{n}$, then we may ask that for all $i=0,1, \ldots, n$ and all $x \in \mathbb{R},\left|D^{i} f(x)-D^{i} g(x)\right|<\varepsilon(x)$.

(ii) If $0 \leq c_{0} \leq c_{1} \leq \ldots$ satisfies $\lim _{i \rightarrow \infty} c_{i}=\infty$ and $g$ is of class $C^{\infty}$, then we may ask that for every $i<\omega$, and each $x \in \mathbb{R}$ such that $|x| \geq c_{i},\left|D^{i} f(x)-D^{i} g(x)\right|<\varepsilon(x)$.

Remark 1.8. The theorem applies to sequences of length less than $\omega_{1}$ as well. For example, to apply it to a single pair of everywhere second category sets $A$ and $B$ of cardinality $\aleph_{1}$, inductively define $A_{\alpha}, \alpha<\omega_{1}$, as follows. The sets $A_{\alpha}$ for $\alpha<\omega$ are any countable family of pairwise disjoint countable dense subsets of $\mathbb{R} \backslash A$. Set $A_{\omega}=A$ and for $\omega<\alpha<\omega_{1}$, take $A_{\alpha}$ to be any translate $A+r$ which is disjoint from all the sets $A_{\beta}, \beta<\alpha$. (Because $2^{\aleph_{0}}>\aleph_{1}$, there is an $r \notin\left\{a_{1}-a_{2}: a_{1} \in \bigcup_{\beta<\alpha} A_{\beta}, a_{2} \in A\right\}$.) Similarly define $B_{\alpha}, \alpha<\omega_{1}$ (with $B_{\omega}=B$ ) and now (b) (or (b) and (c)) applied to these sequences produces the desired order-isomorphism of $A$ and $B$. 
From Theorem 1.7, we can deduce a version of the Barth-Schneider result with the ability to approximate derivatives. (Alternatively, a direct proof of the corollary can be extracted from the proof of the theorem.)

Corollary 1.9. For any two sequences, $\left\langle A_{n}: n<\omega\right\rangle$ and $\left\langle B_{n}: n<\omega\right\rangle$, each consisting of pairwise disjoint countable dense subsets of $\mathbb{R}$, there is an entire function $f: \mathbb{C} \rightarrow \mathbb{C}$ which restricts to an order-isomorphism of $\mathbb{R}$ such that $f\left[A_{n}\right]=B_{n}$ for every $n<\omega$ and $f$ can be chosen to approximate a given nondecreasing surjection as in Theorem 1.7( (c).

Proof. For a given choice of ground model parameters $A_{n}, B_{n}, g, c_{i}$ (thinking of $g$ as a (finite or infinite) sequence of Borel codes $\left\langle g, D g, D^{2} g, \ldots\right\rangle$ for its derivatives), the hypothesized properties of these parameters are $\Pi_{1}^{1}$ and hence continue to hold in the forcing extension which produces the model of Theorem 1.7. (The model is built starting with a model of $V=L$, but it is easily seen (and well known) that the required consequences of $V=L$, namely the existence of diamond sequences on $\omega_{1}$ and on the limits of cofinality $\omega_{1}$ in $\omega_{2}$, can be forced.) The existence of an entire function $f$ satisfying the conclusion is a $\boldsymbol{\Sigma}_{2}^{1}$ property of the parameters and hence holds in the ground model by the Shoenfield absoluteness theorem.

The following problem seems to be open.

Problem 1.10. 1 Are there (in ZFC) two sequences, $\left\langle A_{\alpha}: \alpha<\omega_{1}\right\rangle$ and $\left\langle B_{\alpha}: \alpha<\right.$ $\left.\omega_{1}\right\rangle$, each consisting of pairwise disjoint countable dense subsets of $\mathbb{R}$ and such that for no order-isomorphism $f: \mathbb{R} \rightarrow \mathbb{R}$ do we have $f\left[A_{\alpha}\right]=B_{\alpha}$ for all $\alpha<\omega_{1}$ ?

Note that since every $\aleph_{1}$-dense subset of $\mathbb{R}$ can be partitioned into $\aleph_{1}$ countable dense subsets, in any model for the negative answer to Problem 1.10, any two $\aleph_{1}$ dense subsets of $\mathbb{R}$ are order-isomorphic, i.e., BA holds. In particular, sets of reals of cardinality $\aleph_{1}$ are first category (Remark 1.5). Thus, in part (b) of Theorem 1.7. the restriction that only countably many of the pairs $\left(A_{\alpha}, B_{\alpha}\right)$ consist of countable sets cannot be relaxed to allow for uncountably many such pairs as this would contradict part (a).

Our main tool for approximating differentiable real functions by entire ones is the following strengthening of Carleman's theorem due to Hoischen. It generalizes to entire functions the approximation given in $\mathrm{Wh}$, Lemma 6] (which deals with approximation by analytic functions).

Theorem 1.11 ([ $[\mathrm{Ho}$, Satz $1+$ Satz 2$])$. Let $n<\omega$. If $g: \mathbb{R} \rightarrow \mathbb{R}$ is a function of class $C^{n}$ and $\varepsilon: \mathbb{R} \rightarrow \mathbb{R}$ is a positive continuous function, then there exists an entire function $f$ such that $f[\mathbb{R}] \subseteq \mathbb{R}$ and for all $i=0, \ldots, n$ and all $x \in \mathbb{R}$, $\left|D^{i} f(x)-D^{i} g(x)\right|<\varepsilon(x)$. Furthermore, if $g$ is of class $C^{\infty}$ and $\left\{c_{i}\right\}_{i<\omega}$ is any nondecreasing sequence of nonnegative real numbers with $\lim c_{i}=\infty$, then, for every positive continuous $\varepsilon$ on $\mathbb{R}$ there exists an entire function $f$ such that $f[\mathbb{R}] \subseteq \mathbb{R}$ and for $i=0,1,2 \ldots$ and all $x \in \mathbb{R}$ such that $|x| \geq c_{i},\left|D^{i} f(x)-D^{i} g(x)\right|<\varepsilon(x)$.

(Hoischen doesn't state that $f[\mathbb{R}] \subseteq \mathbb{R}$ but his proof gives this immediately.)

The derivative of a function $f$ is denoted exclusively by $D f$ in this paper. In particular, $f^{\prime}$ does not denote the derivative of $f$. N denotes the set of positive integers. We refer the reader to [Je] or $[\mathrm{Ku}]$ for set-theoretic notation and results

\footnotetext{
${ }^{1}$ S. Shelah has found an elegant example. (Email message 2004/07/06.)
} 
not explained here. We assume that the reader is familiar with the oracle-cc forcing technique as explained in [Sh1998]. We recall the basic definitions and properties.

Definition 1.12. A sequence $\bar{M}=\left\langle M_{\delta}: \delta<\omega_{1}\right\rangle$ is called an oracle if each $M_{\delta}$ is a countable transitive model of a sufficiently large fragment of ZFC, $\delta \in M_{\delta}$ is countable in $M_{\delta}$ and for each $A \subseteq \omega_{1},\left\{\delta: A \cap \delta \in M_{\delta}\right\}$ is stationary in $\omega_{1}$.

The existence of an oracle is equivalent to $\diamond$ (see [ Ku, Theorem II 7.14]) and hence implies $\mathrm{CH}$. Associated with an oracle $\bar{M}$, there is a filter $\operatorname{Trap}(\bar{M})$ generated by the sets

$$
\left\{\delta<\omega_{1}: \delta \text { is a limit ordinal and } A \cap \delta \in M_{\delta}\right\}, \quad A \subseteq \omega_{1} .
$$

This is a proper normal filter containing all closed unbounded sets.

The definition of the $\bar{M}$-chain condition which follows is valid only for partial orders of cardinality $\aleph_{1}$. This case suffices for our purposes.

Definition 1.13. A partial order $P$ satisfies the $\bar{M}$-chain condition, or simply is $\bar{M}$-cc, if there is a one-to-one function $f: P \rightarrow \omega_{1}$ such that the set of limit ordinals $\delta<\omega_{1}$ such that every predense subset of $f^{-1}(\delta)$ of the form $f^{-1}[A]$, where $A \subseteq \delta$ and $A \in M_{\delta}$, is predense in $P$ belongs to $\operatorname{Trap}(\bar{M})$.

It is not hard to verify that if $P$ is $\bar{M}$-cc, then $P$ is ccc. Also, any one-to-one function $g: P \rightarrow \omega_{1}$ can replace $f$ in the definition.

Recall the following properties of oracle-cc forcing. See [Sh1998, Chapter IV] for more details.

Proposition 1.14. Assume $\diamond$. Let $A$ be a second category subset of $\mathbb{R}$. Then there is an oracle $\bar{M}=\left\langle M_{\delta}: \delta\left\langle\omega_{1}\right\rangle\right.$ such that if $P$ is any partial order satisfying the $\bar{M}$-cc, then $\Vdash_{P}$ " $A$ is second category".

Proposition 1.15. The $\bar{M}$-cc satisfies the following properties.

(1) If $\alpha<\omega_{2}$ is a limit ordinal, $\left\langle\left\langle P_{\beta}\right\rangle_{\beta \leq \alpha},\left\langle\dot{Q}_{\beta}\right\rangle_{\beta<\alpha}\right\rangle$ is a finite-support $\alpha$-stage iteration of partial orders, and for each $\beta<\alpha, P_{\beta}$ is $\bar{M}$-cc, then $P_{\alpha}$ is $\bar{M}-c c$.

(2) If $P$ is $\bar{M}$-cc, then there is a $P$-name $\bar{M}^{*}$ for an oracle such that for each $P$-name $\dot{Q}$ for a partial order, if $\Vdash_{P}$ " $\dot{Q}$ is $\bar{M}^{*}$-cc" then $P * \dot{Q}$ is $\bar{M}$-cc.

(3) If $\bar{M}_{\alpha}, \alpha<\omega_{1}$, are oracles, then there is an oracle $\bar{M}$ such that for any partial order $P$, if $P$ is $\bar{M}-c c$, then $P$ is $\bar{M}_{\alpha}$-cc for all $\alpha<\omega_{1}$.

The proof of the main theorem involves many technical arguments for which the intuition may not be clear upon a first reading. The reader may find it helpful to read the proof of Theorem [1.6] upon which the proofs given here build. The proof in Sh1980 is only a brief sketch, but the argument is presented in detail in [BM]. In Section 2, we provide a direct proof of a consequence of the main theorem for which many of the technical difficulties do not arise. Briefly, the difference is that the consequence deals with $C^{\infty}$ functions rather than entire ones and the former are very much more flexible as a class than the latter. In particular, a $C^{\infty}$ function can be zero outside a compact interval without being identically zero. The proof of the main theorem makes no reference to results in Section 2] so the reader who wants to do so can skip that section. We ask the indulgence of the readers of Section 2 for the repetition of some of the arguments and remarks in the proof of the main theorem. 
In Section 3, we show how to reduce Theorem 1.7 to the case where the function $g$ in part (c) is the restriction to $\mathbb{R}$ of an entire function and has a strictly positive derivative. In Section 4, we define the class of entire functions which will be used in the proof of Theorem 1.7 and establish some of its properties. The main lemma needed for the proof of Theorem 1.7 is established in Section 5. The deduction of the theorem from the main lemma is standard oracle-cc technique which we sketch in the final section of the paper.

We thank Ed Bierstone, Ilijas Farah, Paul Gauthier, Ian Graham, Juris Steprans and Bill Weiss for helpful discussions. Thanks are also due to the referee for suggesting the inclusion of the proof of an easier special case of the main theorem as a guide for the reader's intuition.

\section{A SPECIAL CASE}

We begin with a proof of the following special case of the main theorem. Results from this section are not used elsewhere in the paper.

Theorem 2.1. If $Z F C$ is consistent, then so is $Z F C+2^{\aleph_{0}}=\aleph_{2}+$ the following statements.

(a) Every second category set in $\mathbb{R}$ has a second category subset of cardinality $\aleph_{1}$.

(b) For any two everywhere second category sets $A, B \subseteq \mathbb{R}$ of cardinality $\aleph_{1}$, there is a $C^{\infty}$ order-isomorphism $f: \mathbb{R} \rightarrow \mathbb{R}$ such that $f[A]=B$.

We shall use the following standard fact.

Proposition 2.2. Let $f_{n}: \mathbb{R} \rightarrow \mathbb{R}, n \in \mathbb{N}$ be $C^{\infty}$ functions such that for each $i<\omega$, the sequence $\left\{D^{i} f_{n}\right\}_{n=1}^{\infty}$ converges uniformly to a function $g_{i}$. Then $g_{0}$ is a $C^{\infty}$ function and $D^{i} g_{0}=g_{i}$ for each $i<\omega$.

Proof. See [Zi, Example 1.1.10 and Proposition 1.1.13].

We begin by defining a family of $C^{\infty}$ bump functions. We will call an interval $I$ of the form $I=(a, b)$, where $a, b \in \mathbb{Q}$ and $a<b$, a rational interval. Let $\mathcal{I}$ denote the set of all rational intervals.

For $I=(a, b)$ with $a, b \in \mathbb{R}$ and $a<b$, let $g_{I}: \mathbb{R} \rightarrow \mathbb{R}$ be the following $C^{\infty}$ bump function

$$
g_{I}(x)= \begin{cases}e^{-[(x-a)(b-x)]^{-1}} & a<x<b \\ 0 & \text { otherwise }\end{cases}
$$

Let $\mathcal{G}^{*}=\left\{g_{I}: I \in \mathcal{I}\right\}$.

The following proposition provides a means for modifying functions in $\mathcal{G}^{*}$ without significantly altering their derivatives, and also for approximating members of $\mathcal{G}^{*}$ by elements of $\mathcal{G}^{*}$ from a given model.

Proposition 2.3. Let $N$ be an elementary submodel of $H_{\theta}$ for some regular $\theta>\mathfrak{c}$. Let $\varepsilon$ be a positive rational number. Let $i_{0} \in \mathbb{N}$. Let $f_{0}: \mathbb{R} \rightarrow \mathbb{R}$ be the identity function.

Suppose that we are given countable dense subsets $B$ and $C$ of $\mathbb{R}$ with $B, C \in N$. Let $u \in \mathbb{R}$. Let $f \in f_{0}+\operatorname{span} \mathcal{G}^{*}$. Let $K_{0}, K_{1} \subseteq \mathbb{R}$ be finite such that $K_{0}, f\left[K_{1}\right] \in N$. Let $h \subseteq f$ be finite such that $h \in N$. Assume that the sets $\operatorname{dom} h, K_{0}, K_{1},\{u\}$ are pairwise disjoint. Then there is a function $f^{\prime} \in N \cap\left(f_{0}+\operatorname{span} \mathcal{G}^{*}\right)$ such that 
for some rational interval I and positive rational number $r$, the following properties hold:

(a) $h \subseteq f^{\prime}$;

(b) for all $x \in K_{0}, f^{\prime}(x) \in C$;

(c) for all $x \in K_{1}$, there is a $b_{x} \in B$ such that $\left|b_{x}-x\right|<\varepsilon$ and $f^{\prime}\left(b_{x}\right)=f(x)$;

(d) for all $i \leq i_{0}, r\left\|D^{i} g_{I}\right\|_{\infty}<\varepsilon$ and for all $\sigma \in \mathbb{R}$ such that $|\sigma| \leq r$,

$$
\left\|D^{i} f-D^{i}\left(f^{\prime}+\sigma g_{I}\right)\right\|_{\infty}<\varepsilon
$$

(e) $g_{I}(u) \neq 0$ and for some real number $\sigma$ such that $|\sigma|<r$, we have

$$
f(u)=f^{\prime}(u)+\sigma g_{I}(u) .
$$

Proof. Let $K=K_{0} \cup K_{1} \cup$ dom $h$. Choose pairwise disjoint rational intervals $I_{x}$ such that $x \in I_{x}$ for $x \in K \cup\{u\}$. For each $x \in K_{1}$, choose a sequence of points $b_{x, m} \in B \cap I_{x}, m \in \mathbb{N}$, such that $\left|b_{x, m}-x\right|<1 / m$. For each $x \in K_{0}$, choose a sequence of points $c_{x, m} \in C, m \in \mathbb{N}$, such that $\left|c_{x, m}-f(x)\right|<1 / m$. Let

$$
f=f_{0}+\sum_{I \in \mathcal{I}^{\prime}} \lambda_{I} g_{I}
$$

where $\mathcal{I}^{\prime}$ is a finite subset of $\mathcal{I}$ and $\lambda_{I} \in \mathbb{R}$ for each $I \in \mathcal{I}^{\prime}$. Consider functions $f^{\prime}$, $\bar{f}$ of the form

$$
f^{\prime}=f_{0}+\sum_{I \in \mathcal{I}^{\prime}} \mu_{I} g_{I}+\sum_{x \in K} \sigma_{x} g_{I_{x}}
$$

and

$$
\bar{f}=f_{0}+\sum_{I \in \mathcal{I}^{\prime}} \mu_{I} g_{I}+\sum_{x \in K} \sigma_{x} g_{I_{x}}+\sigma g_{I_{u}}=f^{\prime}+\sigma g_{I_{u}},
$$

where $\mu_{I}, \sigma_{x}, \sigma \in \mathbb{R}\left(I \in \mathcal{I}^{\prime}, x \in K\right)$. For $a \in K$, among the functions $g_{I_{a^{\prime}}}, a^{\prime} \in K$, only the one with $a^{\prime}=a$ is not zero at $a$ and for $x \in K_{1}$, only $g_{I_{x}}$ is not zero at $b_{x, m}$. This leads to the following observations.

(1) For $a \in \operatorname{dom} h$ and each choice of $m \in \mathbb{N}$ and $\vec{\mu}=\left(\mu_{I}\right)_{I \in \mathcal{I}^{\prime}}$, there is a unique $\sigma_{a}=\sigma_{a}(m, \vec{\mu})$ for which $f^{\prime}(a)=f(a)=h(a)$, namely

$$
\sigma_{a}(m, \vec{\mu})=\frac{\sum_{I \in \mathcal{I}^{\prime}}\left(\lambda_{I}-\mu_{I}\right) g_{I}(a)}{g_{I_{a}}(a)} .
$$

$\left(\sigma_{a}(m, \vec{\mu})\right.$ does not depend on $m$, but it is convenient to denote it this way for uniformity of the notation.)

(2) For each $x \in K_{0}$ and each choice of $m \in \mathbb{N}$ and $\vec{\mu}=\left(\mu_{I}\right)_{I \in \mathcal{I}^{\prime}}$, there is a unique $\sigma_{x}=\sigma_{x}(m, \vec{\mu})$ for which $f^{\prime}(x)=c_{x, m}$, namely

$$
\begin{aligned}
\sigma_{x}(m, \vec{\mu}) & =\frac{c_{x, m}-f_{0}(x)-\sum_{I \in \mathcal{I}^{\prime}} \mu_{I} g_{I}(x)}{g_{I_{x}}(x)} \\
& =\frac{c_{x, m}-f(x)+\sum_{I \in \mathcal{I}^{\prime}}\left(\lambda_{I}-\mu_{I}\right) g_{I}(x)}{g_{I_{x}}(x)} .
\end{aligned}
$$


(3) For each $x \in K_{1}$ and each choice of $m \in \mathbb{N}$ and $\vec{\mu}=\left(\mu_{I}\right)_{I \in \mathcal{I}^{\prime}}$, there is a unique $\sigma_{x}=\sigma_{x}(m, \vec{\mu})$ for which $f^{\prime}\left(b_{x, m}\right)=f(x)$, namely

$$
\begin{aligned}
\sigma_{x}(m, \vec{\mu}) & =\frac{f(x)-f_{0}\left(b_{x, m}\right)-\sum_{I \in \mathcal{I}^{\prime}} \mu_{I} g_{I}\left(b_{x, m}\right)}{g_{I_{x}}\left(b_{x, m}\right)} \\
& =\frac{f_{0}(x)-f_{0}\left(b_{x, m}\right)+\sum_{I \in \mathcal{I}^{\prime}} \lambda_{I} g_{I}(x)-\sum_{I \in \mathcal{I}^{\prime}} \mu_{I} g_{I}\left(b_{x, m}\right)}{g_{I_{x}}\left(b_{x, m}\right)} .
\end{aligned}
$$

(4) There is a unique value of $\sigma=\sigma(m, \vec{\mu})$ for which $\bar{f}(u)=f(u)$, namely

$$
\sigma(m, \vec{\mu})=\frac{\sum_{I \in \mathcal{I}^{\prime}}\left(\lambda_{I}-\mu_{I}\right) g_{I}(u)}{g_{I_{u}}(u)}
$$

Note that for functions $f^{\prime}$, if $\sigma_{a}=\sigma_{a}(m, \vec{\mu})$ for each $a \in K$, then (a), (b) hold and (c) holds (with $b_{x}=b_{x, m}$ ) if $m$ is large enough. Also, as $\vec{\mu}=\left(\mu_{I}\right)_{I \in \mathcal{I}^{\prime}} \rightarrow\left(\lambda_{I}\right)_{I \in \mathcal{I}^{\prime}}$ and $m \rightarrow \infty$, we have $\sigma_{a}(m, \vec{\mu}) \rightarrow 0$ for each $a \in K$ and $\sigma(m, \vec{\mu}) \rightarrow 0$.

Let $L=[-\ell, \ell]$ be a compact interval such that each of the intervals $I \in \mathcal{I}^{\prime}, I_{a}$ for $a \in K$ and $I_{u}$ are contained in $L$.

Henceforth, we limit ourselves to functions $f^{\prime}, \bar{f}$ for which we have $\sigma_{a}=\sigma_{a}(m, \vec{\mu})$, $a \in K$. Consider the following facts:

(5) for $i \leq i_{0}, D^{i} f(x)=D^{i} \bar{f}(x)\left(=D^{i} f_{0}(x)\right)$ when $x \in \mathbb{R} \backslash L$;

(6) for $i \leq i_{0}$ and $x \in L$, we have

$$
\begin{aligned}
\left|D^{i} f(x)-D^{i} \bar{f}(x)\right| \leq & \sum_{I \in \mathcal{I}^{\prime}}\left|\lambda_{I}-\mu_{I}\right|\left|D^{i} g_{I}(x)\right|+\sum_{a \in K}\left|\sigma_{a}(m, \vec{\mu})\right|\left|D^{i} g_{I_{a}}(x)\right| \\
& +|\sigma|\left|D^{i} g_{I_{u}}(x)\right| \\
\leq & C\left[\sum_{I \in \mathcal{I}^{\prime}}\left|\lambda_{I}-\mu_{I}\right|+\sum_{a \in K}\left|\sigma_{a}(m, \vec{\mu})\right|+|\sigma|\right],
\end{aligned}
$$

where $C=\sup _{x \in L, i \leq i_{0}} \max \left\{\left|D^{i} g_{I}(x)\right|: I \in \mathcal{I}^{\prime} \cup\left\{I_{a}: a \in K\right\} \cup\left\{I_{u}\right\}\right\}$.

We may choose $m \in \mathbb{N}, \mu_{I} \in \mathbb{Q}$ for each $I \in \mathcal{I}^{\prime}$ and $r>0$ so that (d) and (e) are satisfied with $b_{x, m}$ in the place of $b_{x}$ and $I_{u}$ in the place of $I$. (First get a neighborhood of $\vec{\lambda}$, an $m_{0}$ and an $r$ so that (d) is satisfied for $\vec{\mu}$ in the given neighborhood of $\vec{\lambda}$ and $m \geq m_{0}$. Then choose such a $\vec{\mu}$ so that $|\sigma(m, \vec{\mu})|<r$, giving (e).) Note that we have $f^{\prime} \in N$ because $h, H, K_{0}, f\left[K_{1}\right],\left\{b_{x, m}: x \in K_{1}\right\},\left\{c_{x, m}\right.$ : $\left.x \in K_{0}\right\} \in N$, each $\mu_{I}$ is rational (and hence belongs to $N$ ) and each $\sigma_{a}(m, \vec{\mu})$, for $a \in K$, is uniquely determined by the condition that $f^{\prime}(a)=h(a)$ for $a \in \operatorname{dom} h$, $f^{\prime}(x)=c_{x, m}$ for $a=x \in K_{0}$ and $f^{\prime}\left(b_{x, m}\right)=f(x)$ for $a=b_{x, m}\left(x \in K_{1}\right)$, and hence belongs to $N$ by elementarity.

We now proceed to prove the main technical lemma for the oracle-cc iteration. The reader familiar with oracle-cc iteration will see that this lemma completes the proof. In the final section of the paper we have indicated how this standard oraclecc argument proceeds in the case of our main theorem, so we omit it in the present section.

Lemma 2.4. Let $\bar{M}=\left\langle M_{\delta}: \delta<\omega_{1}\right\rangle$ be an oracle. Let $A, B \subseteq \mathbb{R}$ be everywhere second category sets of cardinality $\aleph_{1}$. There is a forcing notion $P$ satisfying the 
$\bar{M}$-cc such that for every $G \subseteq P$ generic over $V, V[G] \models$ there is a $C^{\infty}$ function $f: \mathbb{R} \rightarrow \mathbb{R}$ for which the following properties hold:

(i) $f$ is an order-isomorphism of $\mathbb{R}$;

(ii) $f[A]=B$.

Proof. Let $f_{0}: \mathbb{R} \rightarrow \mathbb{R}$ be the identity map. For the rest of the proof, fix a suitably large regular cardinal $\theta$. Let $\left\langle I_{n}: n<\omega\right\rangle$ list all the nonempty open intervals with rational endpoints. Fix a well-ordering of $\mathbb{R}$ in type $\omega_{1}$. (CH holds because there is an oracle.) Let $Q$ denote the set $\omega_{1} \times \omega \times 2$ equipped with the lexicographical order which we denote by $\triangleleft$. We will inductively define partial orders $P(u), u \in Q$, from the following class of partial orders.

Definition 2.5. Let $N \prec H_{\theta}$. Let $\bar{a}=\left\langle a_{\xi}: \xi<\alpha\right\rangle, \bar{b}=\left\langle b_{\xi}: \xi<\beta\right\rangle$ be one-to-one sequences of real numbers, $\alpha, \beta \leq \omega_{1}$. We write, for $\delta<\omega_{1}$,

$$
\bar{a}^{\delta}=\left\{a_{\omega \delta+n}: n<\omega, \omega \delta+n<\alpha\right\} \quad \text { and } \quad \bar{b}^{\delta}=\left\{b_{\omega \delta+n}: n<\omega, \omega \delta+n<\beta\right\} .
$$

$P(\bar{a}, \bar{b}, N)$ denotes the partial order consisting of conditions $p=\left(h_{p}, f_{p}, \varepsilon_{p}, n_{p}\right)$ such that

(i) $h_{p}$ is a finite partial order-preserving map from $\left\{a_{\xi}: \xi<\alpha\right\}$ to $\left\{b_{\xi}: \xi<\beta\right\}$;

(ii) $h_{p}\left[\bar{a}^{\delta}\right] \subseteq \bar{b}^{\delta}$ for all $\delta<\omega_{1}$;

(iii) $f_{p} \in\left(f_{0}+\operatorname{span} \mathcal{G}^{*}\right) \cap N$;

(iv) $h_{p} \subseteq f_{p}$;

(v) $\left\|D\left(f_{p}-f_{0}\right)\right\|_{\infty}<1-\varepsilon_{p}$;

(vi) $\varepsilon_{p}$ is a rational number, $0<\varepsilon_{p}<1$, and $1 \leq n_{p}<\omega$.

The order is given by $p \leq q$ if and only if

(vii) $h_{p} \supseteq h_{q}, n_{p} \geq n_{q}$;

(viii) for all $i \leq n_{q},\left\|D^{i} f_{p}-D^{i} f_{q}\right\|_{\infty}+\varepsilon_{p} \leq \varepsilon_{q}$. (This gives in particular $\varepsilon_{p} \leq \varepsilon_{q}$.)

The order relation in Definition 2.5 is transitive because if $p \leq q \leq r$, then $h_{p} \supseteq h_{q} \supseteq h_{r}, n_{p} \geq n_{q} \geq n_{r}$ and for $i \leq n_{r}$, we have $i \leq n_{q}$ as well and hence

$$
\begin{aligned}
\left\|D^{i} f_{p}-D^{i} f_{r}\right\|_{\infty}+\varepsilon_{p} & \leq\left\|D^{i} f_{q}-D^{i} f_{r}\right\|_{\infty}+\left(\left\|D^{i} f_{p}-D^{i} f_{q}\right\|_{\infty}+\varepsilon_{p}\right) \\
& \leq\left\|D^{i} f_{q}(z)-D^{i} f_{r}\right\|_{\infty}+\varepsilon_{q} \leq \varepsilon_{r} .
\end{aligned}
$$

Fix a function $\gamma: \omega_{1} \rightarrow 2$ so that for each $i<2,\left|\gamma^{-1}(i)\right|=\aleph_{1}$.

To each $u=(\delta, n, j) \in Q$ we associate a pair of ordinals $(\alpha, \beta)=(\alpha(u), \beta(u))$ as follows. Let $\gamma(\delta)=i$. If $j=0$, let $(\alpha, \beta)$ be the pair of ordinals $(\omega \delta+n, \omega \delta+n)$. If $j=1$, let $(\alpha, \beta)$ be the pair $(\omega \delta+n+1, \omega \delta+n)$ if $i=0$, and $(\omega \delta+n, \omega \delta+n+1)$ if $i=1$.

(Notice that the pair $(\alpha(u), \beta(u))$ uniquely determines $u$ : from $(\alpha, \beta)$ we can clearly recover $\delta$ and $n$, and we have $j=0$ if $\alpha=\beta$ and $j=1$ otherwise.)

We inductively define one-to-one enumerations $\bar{a}=\left\langle a_{\xi}: \xi<\omega_{1}\right\rangle$ of $A$ and $\bar{b}=\left\langle b_{\xi}: \xi<\omega_{1}\right\rangle$ of $B$ and a continuous $\in$-increasing sequence $\left\langle N_{u}:(1,0,0) \unlhd u \in\right.$ $Q\rangle$ of countable elementary submodels of $H_{\theta}$, and then for each $u \in Q$ such that $(1,0,0) \unlhd u$ we define

$$
P(u)=P\left(\bar{a}\left\lceil\alpha(u), \bar{b} \uparrow \beta(u), N_{u}\right) .\right.
$$

We simplify the notation by writing $P(u)=P(\bar{a} \uparrow \alpha(u), \bar{b} \uparrow \beta(u))$, omitting the explicit mention of $N_{u}$. No confusion should arise since $\alpha(u)$ and $\beta(u)$ uniquely determine $u$, and hence $N_{u}$. The indexing of the induction is such that for fixed $\delta<\omega_{1}$ such that $\gamma(\delta)=i$, at stage $u=(\delta, n, j)$ of the induction, if $i=0$, then we 
pick $a_{\omega \delta+n} \in A$ if $j=0$ and $b_{\omega \delta+n} \in B$ if $j=1$, whereas if $i=1$, then we pick $b_{\omega \delta+n} \in B$ if $j=0$ and $a_{\omega \delta+n} \in A$ if $j=1$. In other words, the elements of $\bar{a}^{\delta}$ and $\bar{b}^{\delta}$ are chosen alternately from $A$ and $B$, respectively, starting with an element of $A$ when $i=0$ and with an element of $B$ when $i=1$, as indicated in Tables 1 and 2 .

TABLE 1. Indexing when $i=\gamma(\delta)=0$ :

\begin{tabular}{|c|c|c|c|}
\hline $\begin{array}{c}\text { Stage } \\
u=(\delta, n, j)\end{array}$ & $\begin{array}{c}\text { Element chosen } \\
\text { at stage } u\end{array}$ & $\alpha(u)$ & $\beta(u)$ \\
\hline$(\delta, 0,0)$ & $a_{\omega \delta} \in A$ & $\omega \delta$ & $\omega \delta$ \\
$(\delta, 0,1)$ & $b_{\omega \delta} \in B$ & $\omega \delta+1$ & $\omega \delta$ \\
$(\delta, 1,0)$ & $a_{\omega \delta+1} \in A$ & $\omega \delta+1$ & $\omega \delta+1$ \\
$(\delta, 1,1)$ & $b_{\omega \delta+1} \in B$ & $\omega \delta+2$ & $\omega \delta+1$ \\
$\ldots$ & $\ldots$ & $\ldots$ & $\ldots$ \\
$(\delta, n, 0)$ & $a_{\omega \delta+n} \in A$ & $\omega \delta+n$ & $\omega \delta+n$ \\
$(\delta, n, 1)$ & $b_{\omega \delta+n} \in B$ & $\omega \delta+n+1$ & $\omega \delta+n$ \\
$\ldots$ & $\ldots$ & $\ldots$ & $\ldots$ \\
\hline
\end{tabular}

TABLE 2. Indexing when $i=\gamma(\delta)=1$ :

\begin{tabular}{|c|c|c|c|}
\hline $\begin{array}{c}\text { Stage } \\
u=(\delta, n, j)\end{array}$ & $\begin{array}{c}\text { Element chosen } \\
\text { at stage } u\end{array}$ & $\alpha(u)$ & $\beta(u)$ \\
\hline$(\delta, 0,0)$ & $b_{\omega \delta} \in B$ & $\omega \delta$ & $\omega \delta$ \\
$(\delta, 0,1)$ & $a_{\omega \delta} \in A$ & $\omega \delta$ & $\omega \delta+1$ \\
$(\delta, 1,0)$ & $b_{\omega \delta+1} \in B$ & $\omega \delta+1$ & $\omega \delta+1$ \\
$(\delta, 1,1)$ & $a_{\omega \delta+1} \in A$ & $\omega \delta+1$ & $\omega \delta+2$ \\
$\ldots$ & $\ldots$ & $\ldots$ & $\ldots$ \\
$(\delta, n, 0)$ & $b_{\omega \delta+n} \in B$ & $\omega \delta+n$ & $\omega \delta+n$ \\
$(\delta, n, 1)$ & $a_{\omega \delta+n} \in A$ & $\omega \delta+n$ & $\omega \delta+n+1$ \\
$\ldots$ & $\ldots$ & $\ldots$ & $\ldots$ \\
\hline
\end{tabular}

For technical reasons, we also define a second sequence $\left\langle N_{u}^{\prime}: u \in Q\right\rangle$ of countable elementary submodels of $H_{\theta}$ and functions $e_{\delta}, 1 \leq \delta<\omega_{1}$.

Fix countable dense sets $A_{0} \subseteq A$ and $B_{0} \subseteq B$.

We will arrange that the following conditions hold for all $u=(\delta, n, j) \in Q$, with $\gamma(\delta)=i$.

(1) For $\delta=0$, the only requirements are that $\bar{a}^{0}=A_{0}$ and $\bar{b}^{0}=B_{0}$, where $A_{0}$ and $B_{0}$ are the countable dense sets fixed above (i.e., we define $\left\{a_{n}\right.$ : $n<\omega\}$ and $\left\{b_{n}: n<\omega\right\}$ to be any one-to-one enumerations of $A_{0}$ and $B_{0}$, respectively).

(2) For $\delta \geq 1, N_{u} \in N_{u}^{\prime}$ are countable elementary submodels of $H_{\theta}$.

(3) $f_{0},\left\langle c_{i}: i<\omega\right\rangle, \eta, A_{0}, B_{0}, A, B$ are all elements of $N_{(0,0,0)}$. 
(4) If $\delta \geq 1$ and $(n, j)=(0,0)$, then

(i) $N_{u}=\bigcup\left\{N_{v}: v \triangleleft u\right\}$;

(ii) if $i=0$, then $a_{\omega \delta}$ is the least element of $A \backslash\left\{a_{\xi}: \xi<\omega \delta\right\}$;

(iii) if $i=1$, then $b_{\omega \delta}$ is the least element of $B \backslash\left\{b_{\xi}: \xi<\omega \delta\right\}$.

In (ii) and (iii), "least" refers to the well-ordering of $\mathbb{R}$ fixed earlier.

(5) If $\delta \geq 1$ and $(n, j) \neq(0,0)$, then

(i) $e_{\delta},\left\langle a_{\xi}: \xi<\alpha(u)\right\rangle,\left\langle b_{\xi}: \xi<\beta(u)\right\rangle,\left\langle M_{\delta^{\prime}}: \delta^{\prime} \leq \delta\right\rangle$, and $\left\langle N_{v}: v \triangleleft u\right\rangle$ all belong to $N_{u}$.

(ii) If $i=0, j=0$ or $i=1, j=1$, then $a_{\omega \delta+n} \in A \cap I_{n}$ is a Cohen real over $N_{u}^{\prime}$.

(iii) If $i=0, j=1$ or $i=1, j=0$, then $b_{\omega \delta+n} \in B \cap I_{n}$ is a Cohen real over $N_{u}^{\prime}$.

The point of using $N_{u}^{\prime}$ rather than $N_{u}$ in (ii) and (iii) is that it will be useful later to have $P(u)$ belonging to the model over which the Cohen reals are chosen. (See the paragraphs immediately following the proofs of Claim 2.8 and Claim 2.10,

(6) If $\delta \geq 1, e_{\delta}$ is a bijective map of $P(\bar{a}\lceil\omega \delta, \bar{b}\lceil\omega \delta)$ onto $\omega \delta$.

(7) For each $\delta^{\prime}$ such that $\delta^{\prime}<\delta, e_{\delta^{\prime}} \subseteq e_{\delta}$.

(8) If $\delta \geq 1$, the predense subsets of $P(\bar{a}\lceil\omega \delta, \bar{b}\lceil\omega \delta)$ which have the form $e_{\delta}^{-1}[S]$ for some $S \in \bigcup_{\eta \leq \delta} M_{\eta}$ are predense in $P(\bar{a}\lceil\omega(\delta+1), \bar{b}\lceil\omega(\delta+1))$.

Remark 2.6. (a) From (1) and (5)((ii)+(iii)), it follows that the sets $\bar{a}^{\delta}$ and $\bar{b}^{\delta}$ are dense in $\mathbb{R}$. From $(4)((\mathrm{ii})+(\mathrm{iii}))$ and $(5)((\mathrm{ii})+(\mathrm{iii}))$, we get $\bar{a}^{\delta} \subseteq A$ and $\bar{b}^{\delta} \subseteq B$. From the same clauses together with (5)(i), we get that the enumerations $\left\langle a_{\xi}: \xi<\omega_{1}\right\rangle$ and $\left\langle b_{\xi}: \xi<\omega_{1}\right\rangle$ are one-to-one. (Note that $\alpha(u)=\omega \delta+n$ in (5)(ii) and $\beta(u)=\omega \delta+n$ in (5)(iii).)

(b) From 4(ii) and the fact that $\gamma^{-1}(0)$ is uncountable, it follows that $A=\left\{a_{\xi}\right.$ : $\left.\xi<\omega_{1}\right\}$. Similarly, we get $B=\left\{b_{\xi}: \xi<\omega_{1}\right\}$.

(c) It follows inductively, using (5)(i) at successor stages, that $\left\{a_{\xi}: \xi<\right.$ $\alpha(u)\} \subseteq N_{u}$ and $\left\{b_{\xi}: \xi<\beta(u)\right\} \subseteq N_{u}$. (At a limit stage $u=(\delta, 0,0)$, we have $\alpha(u)=\beta(u)=\omega \delta$. If $\xi<\omega \delta$, then $\xi=\omega \delta^{\prime}+n^{\prime}$ for some $\delta^{\prime}<\delta$ and $n^{\prime}<\omega$. Then $a_{\xi}$ and $b_{\xi}$ are defined (in an order depending on $\left.\gamma\left(\delta^{\prime}\right)\right)$ at stages $\left(\delta^{\prime}, n^{\prime}, 0\right)$ and $\left(\delta^{\prime}, n^{\prime}, 1\right)$. By the induction hypothesis, $a_{\xi}, b_{\xi} \in N_{\left(\delta^{\prime}, n^{\prime}+1,0\right)} \subseteq N_{u}$.) Hence, $h_{p} \in N_{u}$ for each $p \in P(u)$.

(d) From (4)(i) and (5)(i), it follows that the sequence $\left\langle N_{u}:(1,0,0) \unlhd u \in Q\right\rangle$ is $\in$-increasing and continuous at limits. This gives in particular that for each limit ordinal $\delta>\omega, P\left(\bar{a}\left\lceil\omega \delta, \bar{b}\lceil\omega \delta)=\bigcup_{\delta^{\prime}<\delta} P\left(\bar{a}\left\lceil\omega \delta^{\prime}, \bar{b}\left\lceil\omega \delta^{\prime}\right)\right.\right.\right.\right.$.

(e) Set $P=P\left(\bar{a}\left\lceil\omega_{1}, \bar{b}\left\lceil\omega_{1}, \bigcup_{u \in Q} N_{u}\right)\right.\right.$. In the third coordinate we could put the universe (more precisely, $H_{\theta}$ ) since $\bigcup_{u \in Q} N_{u}$ includes all $C^{\infty}$ functions by (5)(i) and the assumption on the $M_{\delta}$ 's. The conditions (6)-(8) ensure that $P$ is $\bar{M}$-cc. To see this, let $e=\bigcup_{\omega \leq \delta<\omega_{1}} e_{\delta}: P \rightarrow \omega_{1}$. For any infinite $\delta<\omega_{1}$ we have $e^{-1}[\omega \delta]=P(\bar{a}\lceil\omega \delta, \bar{b}\lceil\omega \delta)$ and for each $S \subseteq \omega \delta$ belonging to $M_{\delta}$, whenever a set $E$ of the form $e^{-1}[S]=e_{\delta}^{-1}[S]$ is predense in $P(\bar{a} \backslash \omega \delta, \bar{b} \backslash \omega \delta)$, a simple induction on $\delta^{\prime}$ using (8) shows that if $\delta \leq \delta^{\prime}<\omega_{1}$, then $E$ is predense in $P\left(\bar{a}\left\lceil\omega \delta^{\prime}, \bar{b}\left\lceil\omega \delta^{\prime}\right)\right.\right.$. Thus, $E$ is predense in $P$. For a club of $\delta<\omega_{1}$ we have $\omega \delta=\delta$, so this shows that $P$ satisfies the $\bar{M}$-cc. 
We begin by arranging (1)-(7) by induction on $u=(\delta, n, j)$. This is straightforward and we leave most of it to the reader. Notice that because the reals $a_{\xi}$ and $b_{\xi}$ being constructed are not indexed directly by $u$, we need to check that clauses (4)((ii)+(iii)), (5) and (6) make sense. For 5(ii) for example, it is important that at stage $u, a_{\omega \delta+n}$ has not yet been defined. But at an earlier stage $v=\left(\delta^{\prime}, n^{\prime}, j^{\prime}\right)$, we defined $a_{\omega \delta^{\prime}+n^{\prime}}$ or $b_{\omega \delta^{\prime}+n^{\prime}}$. If $\left(\delta^{\prime}, n^{\prime}\right)$ lexicographically precedes $(\delta, n)$, then $\omega \delta^{\prime}+n^{\prime}<\omega \delta+n$. If $\left(\delta^{\prime}, n^{\prime}\right)=(\delta, n)$, then necessarily $\left(j^{\prime}, j\right)=(0,1)$. Since $j=1$, the assumption of 5(ii) gives $i=1$. So at stage $v$ we defined $b_{\omega \delta+n}$, not $a_{\omega \delta+n}$. A similar argument holds for 5(iii) and (4)((ii)+(iii)). Similarly, we can check that in (5)(i), $a_{\xi}$ for $\xi<\alpha(u)$ and $b_{\xi}$ for $\xi<\beta(u)$ were defined before stage $u$. For (6), we observe that the partial order $P(\bar{a}\lceil\omega \delta, \bar{b}\lceil\omega \delta)$ is defined because we have reached or passed the stage $(\delta, 0,0)$ and $N_{(\delta, 0,0)}$ has been defined. The function $e_{\delta}$ is chosen at stage $(\delta, 0,0)$. By Remark 2.6(d), the choice of $e_{\delta}$ is dictated by $(7)$ when $\delta>\omega$ is a limit ordinal. The function $e_{\delta+1}$ can be taken to be an arbitrary extension of $e_{\delta}$ satisfying (6).

We must check that the construction gives (8). Let $E$ be a predense subset of $P\left(\bar{a}\left\lceil\omega \delta, \bar{b}\lceil\omega \delta)\right.\right.$ of the appropriate form, i.e., $E=e_{\delta}^{-1}[S]$ for some $S \in \bigcup_{\eta \leq \delta} M_{\eta}$. We will show by induction on $u=(\delta, n, j) \in Q$ such that $(\delta, 0,0) \unlhd u \triangleleft(\delta+1,0,0)$ that $E$ remains predense in $P(u+1)$, where $u+1$ denotes the successor of $u$ in $Q$, i.e., $(\delta, n, 1)$ if $j=0$ and $(\delta, n+1,0)$ if $j=1$. (This establishes (8) since each member of $P(\bar{a}\lceil\omega(\delta+1), \bar{b}\lceil\omega(\delta+1))$ belongs to $P(\bar{a}\lceil\omega \delta+n, \bar{b}\lceil\omega \delta+n)$ for some $n<\omega$.)

Remark 2.7. At the stage where $n=0$ and $j=0$, we consider the passage from $P(\bar{a}\lceil\omega \delta, \bar{b}\lceil\omega \delta)$ to either $P(\bar{a}\lceil\omega \delta+1, \bar{b}\lceil\omega \delta)$ or $P(\bar{a}\lceil\omega \delta, \bar{b}\lceil\omega \delta+1)$ (depending on whether $i=0$ or $i=1)$. These two partial orders have the same allowable finite parts $h_{p}$ for their conditions because, by Definition 2.5(ii), there is no legal value for either of $a_{\omega \delta}$ or $b_{\omega \delta}$ to correspond to until the other is chosen.

Let $p \in P(u+1) \backslash P(u)$. We must show that $p$ is compatible with some member of E.

Case 1. $h_{p} \in N_{u}$.

Proposition 2.3 gives a function $f^{\prime} \in\left(f_{0}+\operatorname{span} \mathcal{G}^{*}\right) \cap N_{u}$ such that

1(a) $h_{p} \subseteq f^{\prime}$

1 (d) for all $i \leq n_{p},\left\|D^{i} f_{p}-D^{i} f^{\prime}\right\|_{\infty}<\frac{1}{2} \varepsilon_{p}$ (and hence $\left\|D\left(f^{\prime}-f_{0}\right)\right\|_{\infty}<1-\frac{1}{2} \varepsilon_{p}$ ).

The letters in the labels here correspond to those in the statement of Proposition 2.3 The number 1 is a reference to Case 1 . We will use similar notation in the rest of the proof when applying this proposition. Then

$$
q=\left(h_{p}, f^{\prime}, \varepsilon_{p} / 2, n_{p}\right)
$$

belongs to $P(u)$. Also, $q$ and some $r \in E$ have a common extension $q^{\prime} \in P(u)$. Then $q^{\prime} \leq p$ since for each $i \leq n_{p}$, we have also $i \leq n_{q}$ (since $n_{q}=n_{p}$ ) and hence

$$
\begin{aligned}
\left\|D^{i} f_{q^{\prime}}-D^{i} f_{p}\right\|_{\infty}+\varepsilon_{q^{\prime}} & \leq\left\|D^{i} f_{q}-D^{i} f_{p}\right\|_{\infty}+\left\|D^{i} f_{q^{\prime}}-D^{i} f_{q}\right\|_{\infty}+\varepsilon_{q^{\prime}} \\
& =\left\|D^{i} f^{\prime}-D^{i} f_{p}\right\|_{\infty}+\left\|D^{i} f_{q^{\prime}}-D^{i} f_{q}\right\|_{\infty}+\varepsilon_{q^{\prime}} \\
& \leq \varepsilon_{p} / 2+\left\|D^{i} f_{q^{\prime}}-D^{i} f_{q}\right\|_{\infty}+\varepsilon_{q^{\prime}} \\
& \leq \varepsilon_{p} / 2+\varepsilon_{q}=\varepsilon_{p} .
\end{aligned}
$$


Case 2. $h_{p} \notin N_{u}$.

By Remark 2.7, we have $(n, j) \neq(0,0)$. Hence (by (5)(i)), $e_{\delta},\left\langle M_{\delta^{\prime}}: \delta^{\prime} \leq \delta\right\rangle \in N_{u}$ which gives $S \in N_{u}$ and hence $E=e_{\delta}^{-1}[S] \in N_{u}$.

Subcase 2a. $i=0, j=1$ or $i=1, j=0$.

In this subcase, $h_{p}$ has the form $h \cup\left\{\left(a, b_{\omega \delta+n}\right)\right\}$ for some $h \subseteq N_{u}$ and $a \in$ $\left\{a_{\omega \delta+m}: m<n+1-i\right\}$.

Proposition 2.3 gives a rational interval $I_{1}$, a function $f^{\prime} \in\left(f_{0}+\operatorname{span} \mathcal{G}^{*}\right) \cap N_{u}$ and a rational number $\lambda_{0}>0$ such that

2a(a) $h \subseteq f^{\prime}$

2a(d) for all $i \leq n_{p}$ we have $\lambda_{0}\left\|D^{i} g_{I_{1}}\right\|_{\infty}<\varepsilon_{p} / 4$ and for all $\lambda \in \mathbb{R}$ such that $|\lambda| \leq \lambda_{0}$

$$
\left\|D^{i} f_{p}-D^{i}\left(f^{\prime}+\lambda g_{I_{1}}\right)\right\|_{\infty}<\frac{1}{2} \varepsilon_{p}
$$

(and hence in particular $\left.\left\|D\left(\left(f^{\prime}+\lambda g_{I_{1}}\right)-f_{0}\right)(x)\right\|<1-\frac{1}{2} \varepsilon_{p}\right)$;

2a(e) $g_{I_{1}}(a) \neq 0$ and for some number $\lambda$ such that $|\lambda|<\lambda_{0}$, we have $h_{p} \subseteq$ $f^{\prime}+\lambda g_{I_{1}}$.

For functions $g_{0}: \mathbb{R} \rightarrow \mathbb{R}$, numbers $\mu>0$, and rational intervals $I$, define

$$
V\left(g_{0}, \mu, I\right)=\left\{\left(g_{0}+\lambda g_{I}\right)(a):|\lambda|<\mu\right\} .
$$

As long as $g_{I}(a) \neq 0, V\left(g_{0}, \mu, I\right)$ is a nonempty open interval in $\mathbb{R}$. Consider the open interval $U=V\left(f^{\prime}, \lambda_{0}, I_{1}\right)$. By (e), $f_{p}(a)=b_{\omega \delta+n} \in U$. Define

$$
q_{0}=\left(h, f^{\prime}, \varepsilon_{p} / 2, n_{p}\right)
$$

and notice that $q_{0} \in P(u)$.

The idea now is to find a common extension of $p$ and an element of $E$ by taking instead a common extension of $q_{0}$ and an element of $E$. The latter exists by the induction hypothesis since $q_{0} \in P(u)$. Unfortunately, such an extension might not even be compatible with $p$. Claim 2.8 establishes that the common extensions of $q_{0}$ and an element of $E$ exist in sufficient profusion that one of them must be compatible with $p$. (The reader who has read Shelah's original argument will recognize that this is a version of the argument in [Sh1980, Case 3, p. 565] (or see [BM, Claim 4.7]) adapted to the present context.)

Claim 2.8. The union of the open sets $V\left(f_{q}, \mu, I\right)$ such that

(1) $q \in P(u)$ is a common extension of $q_{0}$ and an element of $E$,

(2) $g_{I}(a) \neq 0$ (i.e., $a \in I$ ),

(3) $\mu>0$ is rational, $m \in \mathbb{N}, I$ is a rational interval,

(4) $V\left(f_{q}, \mu, I\right) \subseteq U$,

(5) for all $i \leq n_{q}, \mu\left\|D^{i} g_{I}\right\|_{\infty} \leq \frac{1}{2} \varepsilon_{q}$

(and hence for all $\lambda$ such that $|\lambda| \leq \mu$ we have

$$
\left.\left\|D^{i}\left(\left(f_{q}+\lambda g_{I}\right)-f_{0}\right)\right\|_{\infty}<1-\frac{1}{2} \varepsilon_{q}\right)
$$

is dense in $U$.

Proof of Claim 2.8, Fix $\lambda_{1} \in \mathbb{Q}$ such that $\left|\lambda_{1}\right|<\lambda_{0}$. Define

$$
w=\bar{f}^{\prime}(a) \in U, \text { where } \bar{f}^{\prime}=f^{\prime}+\lambda_{1} g_{I_{1}} .
$$

Note that the numbers $w$ of this form, as $\lambda_{1}$ runs over all rational numbers such that $\left|\lambda_{1}\right|<\lambda_{0}$, are dense in $U$. Fix $\delta>0$ such that $(w-\delta, w+\delta) \subseteq U$. Let $x_{1}, x_{2} \in A_{0} \backslash \operatorname{dom} h$ satisfy $x_{1}<a<x_{2}$ and $\bar{f}^{\prime}\left(x_{1}\right), \bar{f}^{\prime}\left(x_{2}\right) \in(w-\delta, w+\delta)$. Apply 
Proposition 2.3 to get a function $f^{\prime \prime} \in\left(f_{0}+\operatorname{span} \mathcal{G}^{*}\right) \cap N_{u}$ and a rational number $\lambda_{2}>0$ such that

2.8(a) $h \subseteq f^{\prime \prime}$,

2.8(b) $y_{1}=f^{\prime \prime}\left(x_{1}\right)$ and $y_{2}=f^{\prime \prime}\left(x_{2}\right)$ are both members of $B_{0}$,

$2.8(\mathrm{~d})_{1} y_{1}, y_{2} \in(w-\delta, w+\delta)$.

(This holds as long as the quantities $\left|\left(\bar{f}^{\prime}-f^{\prime \prime}\right)\left(x_{j}\right)\right|, j=1,2$, are small enough.)

$2.8(\mathrm{~d})_{2}$ For all $i \leq n_{p}$,

$$
\left\|D^{i} \bar{f}^{\prime}-D^{i} f^{\prime \prime}\right\|_{\infty}<\frac{1}{8} \varepsilon_{p}
$$

(and hence in particular $\left.\left\|D\left(f^{\prime \prime}-f_{0}\right)\right\|_{\infty}<1-\frac{1}{4} \varepsilon_{p}\right)$.

Note that $f^{\prime \prime}$ is increasing (by 2.8 $(\mathrm{d})_{2},\left\|D f^{\prime \prime}-D f_{0}\right\|_{\infty}<1$ and hence $D f^{\prime \prime}>0$ ) and hence we have $y_{1}<y_{2}$. Then $q_{1} \in P(u)$, where

$$
q_{1}=\left(h \cup\left\{\left(x_{1}, y_{1}\right),\left(x_{2}, y_{2}\right)\right\}, f^{\prime \prime}, \varepsilon_{p} / 8, n_{p}\right) .
$$

Also, $q_{1}$ extends $q_{0}$ because if $i \leq n_{p}$, then

$$
\begin{aligned}
\left\|D^{i} f_{q_{1}}-D^{i} f_{q_{0}}\right\|_{\infty}+\varepsilon_{q_{1}} & =\left\|D^{i} f^{\prime \prime}-D^{i} f^{\prime}\right\|_{\infty}+\varepsilon_{p} / 8 \\
& \leq\left\|D^{i} f^{\prime \prime}-D^{i} \bar{f}^{\prime}\right\|_{\infty}+\left\|D^{i} \bar{f}^{\prime}-D^{i} f^{\prime}\right\|_{\infty}+\varepsilon_{p} / 8 \\
& \leq\left\|D^{i} \bar{f}^{\prime}-D^{i} f^{\prime}\right\|_{\infty}+\varepsilon_{p} / 4 \leq \varepsilon_{p} / 2=\varepsilon_{q_{0}} .
\end{aligned}
$$

By the induction hypothesis, there is a common extension $q \in P(u)$ of $q_{1}$ and some $r \in E$. Since $a$ does not belong to the domains of $h_{q_{1}}$ or $h_{r}(r \in E \subseteq P(\delta, 0,0)$ and hence $\left.\operatorname{dom} h_{r} \subseteq\left\{a_{\xi}: \xi<\omega \delta\right\}\right)$, we may discard it from the domain of $h_{q}$ if necessary to get $a \notin \operatorname{dom} h_{q}$. Choose a rational interval $I$ such that $a \in I$ and $I \cap \operatorname{dom} h_{q}=\emptyset$. For $\mu>0$ small enough we have that part 5 of the claim holds.

Then the functions in the definition of $V\left(f_{q}, \mu, I\right)$ are increasing on $\mathbb{R}$. Since $\left\{\left(x_{1}, y_{1}\right),\left(x_{2}, y_{2}\right)\right\} \subseteq h_{q}$ and $I \cap \operatorname{dom} h_{q}=\emptyset$, we have $V\left(f_{q}, \mu, I\right) \subseteq\left(y_{1}, y_{2}\right) \subseteq$ $(w-\delta, w+\delta) \subseteq U$ and, in particular, part 4 of the claim holds.

This proves Claim 2.8

The dense open subset of $U$ given by Claim 2.8 is coded in $N_{u}^{\prime}$. (As noted earlier, $P(u) \in N_{u}^{\prime}$ when $u$ is not a limit stage.) By (5)(iii), there are $q, \mu, I$ satisfying Claim 2.8(1-5) for which $b_{\omega \delta+n} \in V\left(f_{q}, \mu, I\right)$. Choosing $\lambda$ with $|\lambda|<\mu$ so that $\left(f_{q}+\lambda g_{I}\right)(a)=b_{\omega \delta+n}$, we get that

$$
q^{\prime}=\left(h_{q} \cup\left\{\left(a, b_{\omega \delta+n}\right)\right\}, f_{q}+\lambda g_{I}, \varepsilon_{q} / 2, n_{q}\right)
$$

belongs to $P(u+1)$ (using clause 5 of Claim 2.8) and extends both $q$ and $p$. It extends $q$ by clause 5 of Claim 2.8. To see that $q^{\prime} \leq p$, note that for each $i \leq n_{p}$,

$$
\begin{aligned}
\left\|D^{i} f_{q^{\prime}}-D^{i} f_{p}\right\|_{\infty}+\varepsilon_{q^{\prime}} & \leq\left\|D^{i} f_{q}-D^{i} f_{p}\right\|_{\infty}+\left\|D^{i} f_{q^{\prime}}-D^{i} f_{q}\right\|_{\infty}+\varepsilon_{q^{\prime}} \\
& \leq\left\|D^{i} f_{q}-D^{i} f_{p}\right\|_{\infty}+\varepsilon_{q} \\
& \leq\left\|D^{i} f_{q_{0}}-D^{i} f_{p}\right\|_{\infty}+\left\|D^{i} f_{q}-D^{i} f_{q_{0}}\right\|_{\infty}+\varepsilon_{q} \\
& \leq\left\|D^{i} f_{q_{0}}-D^{i} f_{p}\right\|_{\infty}+\varepsilon_{q_{0}} \\
& \leq \varepsilon_{p} / 2+\varepsilon_{p} / 2=\varepsilon_{p} .
\end{aligned}
$$

Thus, $p$ is compatible with $q$ and hence with some element of $E$.

Subcase $2 \mathrm{~b}$. $i=0, j=0$ or $i=1, j=1$.

In this subcase, $h_{p}$ has the form $h \cup\left\{\left(a_{\omega \delta+n}, b\right)\right\}$ for some $h \subseteq N_{u}$ and $b \in$ $\left\{b_{\omega \delta+m}: m<n+i\right\}$. 
Proposition 2.3 gives a rational interval $I_{1}$, a function $f^{\prime} \in\left(f_{0}+\operatorname{span} \mathcal{G}^{*}\right) \cap N_{u}$ and a rational number $\lambda_{0}>0$ such that

$2 \mathrm{~b}$ (a) $h \subseteq f^{\prime}$;

$2 \mathrm{~b}(\mathrm{~d})$ for all $i \leq n_{p}, \lambda_{0}\left\|D^{i} g_{I_{1}}\right\|_{\infty}<\frac{1}{4} \varepsilon_{p}$ and for all $\lambda \in \mathbb{R}$ such that $|\lambda| \leq \lambda_{0}$,

$$
\left\|D^{i} f_{p}-D^{i}\left(f^{\prime}+\lambda g_{I_{1}}\right)\right\|_{\infty}<\frac{1}{2} \varepsilon_{p}
$$

(and hence in particular $\left.\left\|D\left(\left(f^{\prime}+\lambda g_{I_{1}}\right)-f_{0}\right)\right\|_{\infty}<1-\frac{1}{2} \varepsilon_{p}\right)$;

$2 \mathrm{~b}(\mathrm{e})$ for some number $\lambda$ such that $|\lambda|<\lambda_{0}$, we have $h_{p} \subseteq f^{\prime}+\lambda g_{I_{1}}$.

(This list agrees with the one for Subcase 2a except for (e).)

Remark 2.9. Note that $2 \mathrm{~b}(\mathrm{~d})$ ensures that for $|\lambda| \leq \lambda_{0}, \frac{1}{2} \varepsilon_{p}<D\left(f^{\prime}+\lambda g_{I_{1}}\right)$ and hence $f^{\prime}+\lambda g_{I_{1}}$ is an order-isomorphism.

For functions $g_{0}: \mathbb{R} \rightarrow \mathbb{R}$, numbers $\mu>0$ and rational intervals, define

$$
W\left(g_{0}, \mu, I\right)=\left\{\left(g_{0}+\lambda g_{I}\right)^{-1}(b):|\lambda|<\mu\right\} .
$$

The definition makes sense if $g_{0}+\lambda g_{I}$ is an order-isomorphism whenever $|\lambda|<\mu$. By Proposition 4.1, $W\left(g_{0}, \mu, I\right)$ is an open interval in $\mathbb{R}$ as long as there is no $a \in \mathbb{R}$ such that $g_{0}(a)=b$ and $g_{I}(a)=0$. When $g_{0}$ is invertible, then only the value $a=g_{0}^{-1}(b)$ is relevant. Consider the open interval $U=W\left(f^{\prime}, \lambda_{0}, I_{1}\right)$. By (e), $a_{\omega \delta+n}=f_{p}^{-1}(b) \in U$. Define

$$
q_{0}=\left(h, f^{\prime}, \varepsilon_{p} / 2, n_{p}\right)
$$

and notice that $q_{0} \in P(u)$.

Claim 2.10. The union of the open sets $W\left(f_{q}, \mu, I\right)$ such that

(1) $q \in P(u)$ is a common extension of $q_{0}$ and an element of $E$;

(2) $g_{I}(a) \neq 0$ where $a=f_{q}^{-1}(b)$;

(3) $\mu>0$ is rational, $I$ is a rational interval;

(4) $W\left(f_{q}, \mu, I\right) \subseteq U$;

(5) for all $i \leq n_{q}, \mu\left\|D^{i} g_{I}\right\|_{\infty} \leq \varepsilon_{q} / 2$

(and hence for all $\lambda$ such that $|\lambda| \leq \mu$,

$$
\left.\left\|D\left(\left(f_{q}+\lambda g_{I}\right)-f_{0}\right)\right\|_{\infty}<1-\frac{1}{2} \varepsilon_{q}\right)
$$

is dense in $U$.

Proof of Claim 2.10. Fix $\lambda_{1} \in \mathbb{Q}$ such that $\left|\lambda_{1}\right|<\lambda_{0}$. Define

$$
w=\left(\bar{f}^{\prime}\right)^{-1}(b) \in U, \text { where } \bar{f}^{\prime}=f^{\prime}+\lambda_{1} g_{I_{1}} .
$$

By Remark 2.9, $\bar{f}^{\prime}$ is an order-isomorphism. Note that $w \notin$ dom $h$ since $b \notin$ range $h$ and $h \subseteq \bar{f}^{\prime}$. Note that the numbers $w$ of the given form, as $\lambda_{1}$ runs over all rational numbers such that $\left|\lambda_{1}\right|<\lambda_{0}$, are dense in $U$. Fix $\delta>0$ such that $(w-\delta, w+\delta) \subseteq$ $U$ and $(w-\delta, w+\delta) \cap \operatorname{dom} h=\emptyset$. Let $y_{1}, y_{2} \in B_{0}$ satisfy $y_{1}<b<y_{2}$ and $\left(\bar{f}^{\prime}\right)^{-1}\left(y_{1}\right),\left(\bar{f}^{\prime}\right)^{-1}\left(y_{2}\right) \in(w-\delta, w+\delta)$. Apply Proposition 2.3 to get a rational interval $I_{2}$, a function $f^{\prime \prime} \in\left(f_{0}+\operatorname{span} \mathcal{G}^{*}\right) \cap N_{u}$ and a rational number $\lambda_{2}>0$ such that

2.10(a) $h \subseteq f^{\prime \prime}$;

2.10(c) $y_{1}=f^{\prime \prime}\left(x_{1}\right)$ and $y_{2}=f^{\prime \prime}\left(x_{2}\right)$, where $x_{1}, x_{2}$ are both members of $A_{0}$ and $x_{1}, x_{2} \in(w-\delta, w+\delta) ;$ 
2.10(d) for all $i \leq n_{p}$,

$$
\left\|D^{i} \bar{f}^{\prime}-D^{i} f^{\prime \prime}\right\|_{\infty}<\frac{1}{8} \varepsilon_{p}
$$

(and hence in particular $\left.\left\|D\left(f^{\prime \prime}-f_{0}\right)\right\|_{\infty}<1-\frac{1}{4} \varepsilon_{p}\right)$.

By 2.10(d), $f^{\prime \prime}$ is increasing and hence $x_{1}<x_{2}$. Then $q_{1} \in P(u)$, where

$$
q_{1}=\left(h \cup\left\{\left(x_{1}, y_{1}\right),\left(x_{2}, y_{2}\right)\right\}, f^{\prime \prime}, \varepsilon_{p} / 8, n_{p}\right) .
$$

Exactly as in the proof of Claim 2.8, $q_{1}$ extends $q_{0}$. By the induction hypothesis, there is a common extension $q \in P(u)$ of $q_{1}$ and some $r \in E$. The number $a=$ $f_{q}^{-1}(b)$ does not belong to the domains of $h_{q_{1}}$ or $h_{r}$.

[From the fact that $f_{q}$ is increasing and hence injective, we see that because $f_{q}\left(x_{i}\right)=$ $y_{i}, i=1,2$, and $f_{q}(a)=b$, we have $a \neq x_{i}, i=1,2$. Because $h \subseteq f_{q}$ and $b \notin$ range $h$, we have $a \notin \operatorname{dom} h$. Thus, $a \notin \operatorname{dom} h_{q_{1}}$. Since $h_{r} \subseteq h_{q} \subseteq f_{q}$, if $a$ were in dom $h_{r}$, then $h_{r}(a)=b$, which is not possible because $r \in E \subseteq P(\delta, 0,0)$ and hence range $h_{r} \subseteq\left\{b_{\xi}: \xi<\omega \delta\right\}$.]

We may thus discard it from the domain of $h_{q}$ if necessary to get $a \notin \operatorname{dom} h_{q}$. Choose a rational interval $I$ such that $a \in I$ and $I \cap \operatorname{dom} h_{q}=\emptyset$. As in the proof of Claim 2.8, for $\mu>0$ small enough we have that part 5 of the claim holds. Then the functions in the definition of $W\left(f_{q}, \mu, I\right)$ are order-isomorphisms. Since $\left\{\left(x_{1}, y_{1}\right),\left(x_{2}, y_{2}\right)\right\} \subseteq h_{q}$ and $I \cap \operatorname{dom} h_{q}=\emptyset$, we have $W\left(f_{q}, \mu, I\right) \subseteq\left(x_{1}, x_{2}\right) \subseteq$ $(w-\delta, w+\delta) \subseteq U$ and in particular part 4 of the claim holds.

This proves Claim 2.10.

The dense open subset of $U$ given by Claim 2.10 is coded in $N_{u}^{\prime}$. By (5)(ii), there are $q, \mu, I$ satisfying Claim 2.10(1-5) for which $a_{\omega \delta+n} \in W\left(f_{q}, \mu, I\right)$. Choosing $\lambda$ with $|\lambda|<\mu$ so that $\left(f_{q}+\lambda g_{I}\right)\left(a_{\omega \delta+n}\right)=b$, we get, as in Subcase 2a, that

$$
q^{\prime}=\left(h_{q} \cup\left\{\left(a_{\omega \delta+n}, b\right)\right\}, f_{q}+\lambda g_{I}, \varepsilon_{q} / 2, n_{q}\right)
$$

belongs to $P(u+1)$ and extends both $q$ and $p$. Thus, $p$ is compatible with $q$ and hence with some element of $E$. This completes the proof of (8).

We now have an $\bar{M}$-cc partial order $P=P\left(\bar{a}\left\lceil\omega_{1}, \bar{b}\left\lceil\omega_{1}\right)\right.\right.$ as in Remark 2.6(e). It remains to check that forcing with $P$ adds the desired entire function $f$. Let $h=\bigcup\left\{h_{p}: p \in G\right\}$. For each $x \in A$, it follows easily using Proposition 2.3(b) that conditions with $f_{p}(x) \in B$ are dense and then (by extending such conditions further) so are conditions with $x \in \operatorname{dom} h_{p}$. Similarly, for each $y \in B$, the conditions with $y \in$ range $h_{p}$ are dense (using Proposition 2.3(c) this time). Hence dom $h=A$, range $h=B$ and $h$ is clearly an order-isomorphism. For $k \in \mathbb{N}$, choose $p_{k} \in G$ such that $p_{k+1} \leq p_{k}, n_{p_{k}} \geq k$ and $\varepsilon_{p_{k}}<1 / k$. The sequences $\left\{D^{i} f_{p_{k}}\right\}_{k=1}^{\infty}$ are uniformly Cauchy because for all $k, \ell$ with $k<\ell$ and $k$ large enough so that $i \leq n_{k}$, the fact that $p_{\ell} \leq p_{k}$ gives $\left\|D^{i} f_{p_{\ell}}-D^{i} f_{p_{k}}\right\|_{\infty} \leq \varepsilon_{p_{k}}<1 / k$. Define $f: \mathbb{R} \rightarrow \mathbb{R}$ by $f(x)=\lim _{k \rightarrow \infty} f_{p_{k}}(x)$. For all $i<\omega$, we have $D^{i} f(x)=\lim _{k \rightarrow \infty} D^{i} f_{p_{k}}(x)$ by Proposition 2.2. Also, for each $a \in A$, we can choose $k$ and $p \in G$ such that $p \leq p_{k}$ and $a \in \operatorname{dom} h_{p}$. Then

$$
\left|f_{p_{k}}(a)-h(a)\right|=\left|f_{p_{k}}(a)-h_{p}(a)\right|=\left|f_{p_{k}}(a)-f_{p}(a)\right| \leq \varepsilon_{p_{k}}<1 / k,
$$

and hence $f(a)=\lim _{k \rightarrow \infty} f_{p_{k}}(a)=h(a)$.

This completes the proof of the lemma. 


\section{REDUCTION TO THE APPROXIMATION OF ENTIRE FUNCTIONS}

The results of this section are elementary and are surely known, at least in some form. We sketch the proofs for completeness.

Proposition 3.1. For any interval $[a, b], a<b$, and for any $\varepsilon>0$, there is $a C^{\infty}$ bump function which is zero outside $[a, b]$, positive on $(a, b)$ and whose derivative has a unique zero in $(a, b)$ inside the interval $(b-\varepsilon, b)$.

Proof. For $n \in \mathbb{N}$, the unique zero in $(a, b)$ of the derivative of $x \mapsto$ $\exp \left(-\left[(x-a)^{n}(b-x)\right]^{-1}\right)$ occurs at $\frac{1}{n+1} a+\frac{n}{n+1} b$.

The statements of Proposition 3.2 and Proposition 3.5 hold with $C^{1}$ replaced by $C^{\infty}$ but the $C^{1}$ case suffices for us.

Proposition 3.2. Let $a \in \mathbb{R}$. Let $g: \mathbb{R} \rightarrow \mathbb{R}$ be a continuous function such that $D g(x)>0$ and $D^{2} g(x)=0$ for all $x \neq a$. Let $U$ be an open neighborhood of the point $(a, g(a))$ in $\mathbb{R}^{2}$. There is a $C^{1}$ function $f: \mathbb{R} \rightarrow \mathbb{R}$ such that $f \subseteq g \cup U$ and $D f(x)>0$ for all $x \in \mathbb{R}$.

Proof. This is a calculus exercise. We may assume $a=0$ and $g(0)=0$. Write $g=g_{1}+g_{2}$ where $g_{1}(x)=\min (g(x), 0)$ and $g_{2}(x)=\max (g(x), 0)$. For some $c_{i}>0$, $i=1,2$, we have $g_{1}(x)=c_{1} x$ for all $x \leq 0$ and $g_{2}(x)=c_{2} x$ for all $x \geq 0$. We may assume that $U$ is an open square centered at $(0,0)$. It is enough to find an $f$ which works for $U=(-R, R) \times(-R, R)$ for some $R>0$, because then given a smaller neighborhood $U_{0}=(-r, r) \times(-r, r)$, the function $f_{0}(x)=(r / R) f(R x / r)$ works for $U_{0}$. Let $f_{1}$ be obtained from $g_{1}$ by replacing the portion of the graph over the interval $\left[-\frac{1}{4} c_{1}, \frac{1}{4} c_{1}\right]$ by $x \mapsto-\left(x-\frac{1}{4} c_{1}\right)^{2}$. Let $f_{2}$ be obtained from $g_{2}$ by replacing the portion of the graph over the interval $\left[-\frac{1}{4} c_{2}, \frac{1}{4} c_{2}\right]$ by $x \mapsto\left(x+\frac{1}{4} c_{2}\right)^{2}$. Then $f=f_{1}+f_{2}$ is as desired.

Proposition 3.3. Let $f: \mathbb{R} \rightarrow \mathbb{R}$ be a nondecreasing $C^{1}$ surjection. Let $\varepsilon: \mathbb{R} \rightarrow \mathbb{R}$ be a positive continuous function. There is a $C^{1}$ order-isomorphism $g: \mathbb{R} \rightarrow \mathbb{R}$ having a strictly positive derivative and such that for all $x \in \mathbb{R},|f(x)-g(x)|<\varepsilon(x)$ and $|D f(x)-D g(x)|<\varepsilon(x)$. If $f$ is moreover a $C^{n}$ function, then we may also ask that $g$ be a $C^{n}$ function and that for all $i \leq n$ and all $x \in \mathbb{R},\left|D^{i} f(x)-D^{i} g(x)\right|<$ $\varepsilon(x)$. If $f$ is moreover $C^{\infty}$ and $0 \leq c_{0} \leq c_{1} \leq \ldots$ satisfies $\lim _{i \rightarrow \infty} c_{i}=\infty$, then we may ask that $g$ be $C^{\infty}$ and that for all $i<\omega$ and all $x \in \mathbb{R}$ such that $|x| \geq c_{i}$, $\left|D^{i} f(x)-D^{i} g(x)\right|<\varepsilon(x)$.

Proof. Because $\lim _{x \rightarrow \infty} f(x)=\infty$ and $\lim _{x \rightarrow-\infty} f(x)=-\infty$, we can find a strictly increasing sequence of real numbers $\left\langle a_{i}: i \in \mathbb{Z}\right\rangle$ such that $\lim _{i \rightarrow \infty} a_{i}=\infty$, $\lim _{i \rightarrow-\infty} a_{i}=-\infty$ and $D f\left(a_{i}\right)>0$ for each $i \in \mathbb{Z}$. Since $D f$ is continuous, we can pick positive numbers $\delta_{i}$ such that the intervals $\left[a_{i}-\delta_{i}, a_{i}+\delta_{i}\right], i \in \mathbb{Z}$, are pairwise disjoint and $D f$ is positive everywhere on $\left[a_{i}-\delta_{i}, a_{i}+\delta_{i}\right]$. For each $i \in \mathbb{Z}$, fix a $C^{\infty}$ bump function $g_{i}$ which is zero outside $\left[a_{i}, a_{i+1}\right]$, positive on $\left(a_{i}, a_{i+1}\right)$ and whose derivative has a unique zero in $\left(a_{i}, a_{i+1}\right)$ inside the interval $\left(a_{i+1}-\delta_{i+1}, a_{i+1}\right)$. (Use Proposition 3.1) Hence, $g_{i}$ has a strictly positive derivative on $\left(a_{i}, a_{i+1}-\delta_{i+1}\right)$. We shall take $g=f+\sum_{i \in \mathbb{Z}} \lambda_{i} g_{i}$ for suitably small positive numbers $\lambda_{i}$. Since $D f$ is positive everywhere on $\left[a_{i+1}-\delta_{i+1}, a_{i+1}+\delta_{i+1}\right]$, it is bounded below by a positive constant on this interval. Thus, if $\lambda_{i}$ is small enough, $f+\lambda_{i} g_{i}$ will have a positive derivative on this interval. Then, on this same interval, we have that 
$g=f+\lambda_{i} g_{i}+\lambda_{i+1} g_{i+1}$ has a positive derivative for any choice of $\lambda_{i+1}>0$. It is clear that the constants $\lambda_{i}$ may be chosen small enough so that, in addition, for all $x \in \mathbb{R}$ we have $|f(x)-g(x)|<\varepsilon(x)$ and $|D f(x)-D g(x)|<\varepsilon(x)$. The modifications to the foregoing argument needed to establish the last two clauses are straightforward and left to the reader.

Proposition 3.4. Let $a, b \in \mathbb{R}$ be such that $a<b$ and let $\varepsilon>0$. Let $f:[a, b] \rightarrow \mathbb{R}$ be a nondecreasing continuous map such that $f(a)<f(b)$. Let $y_{a}$, $y_{b}$ be such that $f(a)<y_{a}<f(a)+\varepsilon, f(b)<y_{b}<f(b)+\varepsilon$ and $y_{a}<y_{b}$. There is a strictly increasing piecewise linear continuous map $g:[a, b] \rightarrow \mathbb{R}$ such that $g(a)=y_{a}, g(b)=y_{b}$ and for all $x \in[a, b], f(x) \leq g(x)<f(x)+\varepsilon$.

Proof. We may assume that for the value of $\lambda \in(0,1)$ for which $y_{b}=f(b)+\lambda \varepsilon$, we also have $y_{a}=f(a)+\lambda \varepsilon$. (Note that because $f(a)<f(b)$, this substitution preserves $y_{a}<y_{b}$.) For, given $g$ a strictly increasing piecewise linear continuous map such that $g(a)=f(a)+\lambda \varepsilon$ and $g(b)=y_{b}=f(b)+\lambda \varepsilon$, we get the desired map by modifying the definition of $g$ near $a$ as follows. If $y_{a}<f(a)+\lambda \varepsilon$, then pick $\delta>0$ such that for $x \in[a, a+\delta]$ we have $f(x)<y_{a}$. Then replace $g\lceil[a, a+\delta]$ by the straight line segment joining $\left(a, y_{a}\right)$ and $(a+\delta, g(a+\delta))$. If $f(a)+\lambda \varepsilon<y_{a}$, then find the unique $a^{\prime}>a$ for which $g\left(a^{\prime}\right)=y_{a} .\left(a^{\prime}\right.$ exists because $g(a)<y_{a}<y_{b}=g(b)$.) For a suitably small $\delta>0$ we have $g\left(a^{\prime}+\delta\right)<f(a)+\varepsilon \leq f(x)+\varepsilon$ for all $x \in[a, b]$. Replace $g\left\lceil\left[a, a^{\prime}+\delta\right]\right.$ by the straight line segment joining $\left(a, y_{a}\right)$ and $\left(a^{\prime}+\delta, g\left(a^{\prime}+\delta\right)\right)$.

Now let $a^{\prime}=\sup \{x \in[a, b]: f(a)=f(x)\}, b^{\prime}=\inf \{x \in[a, b]: f(x)=f(b)\}$. Let $c=\sup A$ where $A=\left\{u \in\left[a^{\prime}, b^{\prime}\right]\right.$ : for all $v \in\left(a^{\prime}, u\right]$, there is a piecewise linear strictly increasing continuous function $h_{v}$ defined on $[a, v]$ satisfying $h_{v}(a)=y_{a}=$ $f(a)+\lambda \varepsilon, h_{v}(v)=f(v)+\lambda \varepsilon$ and $f(x)<h_{v}(x)<f(x)+\varepsilon$ for $\left.x \in[a, v]\right\}$. The straight line segments joining $\left(a, y_{a}\right)$ to $(v, f(v)+\lambda \varepsilon)$ for $a^{\prime}<v<a^{\prime}+\delta$ for small enough $\delta$ show that $c>a^{\prime}$. If $c \geq b^{\prime}$, then for $u \in\left(a^{\prime}, b^{\prime}\right) \cap A$ close enough to $b^{\prime}$ and $v \in\left[b^{\prime}, b\right]$, a function $h_{u}$ as in the definition of $u \in A$ and the straight line segment $k_{u, v}$ joining $(u, f(u)+\lambda \varepsilon)$ to $(v, f(v)+\lambda \varepsilon)\left(=\left(v, y_{b}\right)\right)$ define a function $k=h_{u} \cup k_{u, v}$ satisfying $f(x)<k(x)<f(x)+\lambda \varepsilon$ for $x \in[a, v]$. This shows that $b \in A$ which completes the proof in this case.

Now suppose $c<b^{\prime}$. Let $c^{\prime}=\inf \{x \in[a, c]: f(x)=f(c)\}, c^{\prime \prime}=\sup \{x \in[c, b]$ : $f(c)=f(x)\}$. Note that $a^{\prime}<c^{\prime} \leq c \leq c^{\prime \prime}<b^{\prime}$. For $u \in\left(a^{\prime}, c^{\prime}\right)$ close enough to $c^{\prime}$ and $v \in\left[c^{\prime}, c^{\prime \prime}\right]$, the functions $h_{u} \cup k_{u, v}$ defined as above show that $c^{\prime \prime} \in A$ and hence $c=c^{\prime \prime}$. Then for $u \in\left(a^{\prime}, c\right)$ and $v \in\left(c, b^{\prime}\right)$ both close enough to $c$, the functions $h_{u} \cup k_{u, v}$ show that $A$ contains elements larger than $c$, contradicting the definition of $c$.

Proposition 3.5. Let $f: \mathbb{R} \rightarrow \mathbb{R}$ be a nondecreasing surjection. Let $\varepsilon: \mathbb{R} \rightarrow \mathbb{R}$ be a positive continuous function. There is a $C^{1}$ order-isomorphism $g: \mathbb{R} \rightarrow \mathbb{R}$ such that $D g(x)>0$ and $f(x)<g(x)<f(x)+\varepsilon(x)$ for all $x \in \mathbb{R}$.

Proof. The assumption on $f$ ensures that $f$ is continuous and that $\lim _{x \rightarrow \infty} f(x)=$ $\infty$ and $\lim _{x \rightarrow-\infty} f(x)=-\infty$. By replacing $\varepsilon(x)$ by $\min (\varepsilon(x), 1)$, we may assume that $\varepsilon(x) \leq 1$ for all $x \in \mathbb{R}$. Inductively choose integers $0=n_{0}<n_{1}<n_{2}<\ldots$ such that for each nonnegative integer $i$,

$$
f\left(n_{i}\right)+1<f\left(n_{i+1}\right) .
$$


Then inductively choose integers $0=n_{0}>n_{-1}>n_{-2}>\ldots$ such that the same inequality holds for negative integers $i$ as well. For each integer $i$, choose $y_{i}$ so that

$$
f\left(n_{i}\right)<y_{i}<f\left(n_{i}\right)+\inf _{n_{i-1} \leq x \leq n_{i+1}} \varepsilon(x) .
$$

The choice of the $n_{i}$ 's ensures that we have $y_{i}<y_{i+1}$ for each $i$. For each integer $i$, apply Proposition 3.4 to the interval $\left[n_{i}, n_{i+1}\right]$ with $\varepsilon=\inf _{n_{i} \leq x \leq n_{i+1}} \varepsilon(x)$ and piece together the resulting functions to get a strictly increasing piecewise linear continuous function $g: \mathbb{R} \rightarrow \mathbb{R}$ satisfying $f(x)<g(x)<f(x)+\varepsilon(x)$ for all $x \in \mathbb{R}$. Then modify $g$ to round off the corners of its graph using Proposition 3.2 .

Proposition 3.6. Let $\varepsilon: \mathbb{R} \rightarrow \mathbb{R}$ be a positive continuous function and let $g: \mathbb{R} \rightarrow \mathbb{R}$ be a nondecreasing continuous surjection.

(i) If $n$ is a nonnegative integer and $g$ is $C^{n}$, there is an entire function $f: \mathbb{C} \rightarrow$ $\mathbb{C}$ such that $f[\mathbb{R}] \subseteq \mathbb{R}, f\lceil\mathbb{R}$ is an order-isomorphism of $\mathbb{R}$ with a strictly positive derivative and for all $i=0,1, \ldots, n$ and all $x \in \mathbb{R}, \mid D^{i} f(x)-$ $D^{i} g(x) \mid<\varepsilon(x)$.

(ii) If $0 \leq c_{0} \leq c_{1} \leq \ldots$ is such that $\lim _{i \rightarrow \infty} c_{i}=\infty$ and $g$ is $C^{\infty}$, there is an entire function $f: \mathbb{C} \rightarrow \mathbb{C}$ such that $f[\mathbb{R}] \subseteq \mathbb{R}, f \uparrow \mathbb{R}$ is an orderisomorphism of $\mathbb{R}$ with a strictly positive derivative and for every $i<\omega$, and each $x \in \mathbb{R}$ such that $|x| \geq c_{i},\left|D^{i} f(x)-D^{i} g(x)\right|<\varepsilon(x)$.

Proof. Consider first the case of (i) with $n=0$. By Proposition 3.5 there is a $C^{1}$ order-isomorphism $\bar{g}$ with a strictly positive derivative such that $|\bar{g}(x)-g(x)|<$ $\frac{1}{2} \varepsilon(x)$ for all $x \in \mathbb{R}$. Then by Theorem [1.11, there is an entire function $f$ such that $f[\mathbb{R}] \subseteq \mathbb{R}$ and for all $x \in \mathbb{R}$, we have $|f(x)-\bar{g}(x)|<\frac{1}{2} \varepsilon(x)$ and $|D f(x)-D \bar{g}(x)|<$ $D \bar{g}(x)$ (and hence $D f(x)>0$ ). We may assume that $\varepsilon$ is bounded, so that the fact that $|f(x)-g(x)|<\varepsilon(x)$ ensures that $f$ is surjective.

The case of (i) where $n \geq 1$ and (ii) are handled similarly, using Proposition 3.3 instead of Proposition 3.5.

\section{Preliminary Results}

The main goal of this section is to define a family of entire functions and prove Proposition 4.5. which shows how members of this family can be approximated in smaller models of set theory and perturbed slightly to alter their values at certain points. We begin with a technical fact which will be useful in the next section.

Proposition 4.1. Let $f, g: \mathbb{R} \rightarrow \mathbb{R}$ be continuous functions such that $g$ is bounded. Assume that for some open interval $I$ and every $\lambda \in I, f+\lambda g$ is an orderisomorphism of $\mathbb{R}$. Fix $b \in \mathbb{R}$. Then the function $h: I \rightarrow \mathbb{R}$ given by $h(\lambda)=$ $(f+\lambda g)^{-1}(b)$ is continuous. Moreover, $h$ is either constant or strictly monotonic, with the first alternative happening precisely when there is an $x$ such that $f(x)=b$ and $g(x)=0$.

Proof. Let $\lambda$ and $\lambda_{n}, n \in \mathbb{N}$, be elements of $I$ such that $\lambda_{n} \rightarrow \lambda$ as $n \rightarrow \infty$. We want to show that $h\left(\lambda_{n}\right) \rightarrow h(\lambda)$. Let $x_{n}=h\left(\lambda_{n}\right)$, so that $\left(f+\lambda_{n} g\right)\left(x_{n}\right)=b$. Notice that

$(f+\lambda g)\left(x_{n}\right)=\left(f+\lambda_{n} g\right)\left(x_{n}\right)+\left(\lambda-\lambda_{n}\right) g\left(x_{n}\right)=b+\left(\lambda-\lambda_{n}\right) g\left(x_{n}\right) \rightarrow b$ as $n \rightarrow \infty$ since $g$ is bounded. Since $f+\lambda g$ is an order-isomorphism, it is a homeomorphism and hence $h\left(\lambda_{n}\right)=x_{n} \rightarrow(f+\lambda g)^{-1}(b)=h(\lambda)$ as $n \rightarrow \infty$. This proves the first part of the proposition. 
For the second, note first that if there is an $x_{0}$ such that $f\left(x_{0}\right)=b$ and $g\left(x_{0}\right)=0$, then for any $\lambda \in I$ we have $(f+\lambda g)\left(x_{0}\right)=b$ and hence $h(\lambda)=x_{0}$. Now suppose that there is no such $x_{0}$. Since $h$ is continuous and defined on an interval, if we show that $h$ is one-to-one, then it will follow that $h$ is stricly monotonic. Suppose that for some $\lambda_{1}, \lambda_{2}$ we have $h\left(\lambda_{1}\right)=h\left(\lambda_{2}\right)$, i.e., letting $x=h\left(\lambda_{1}\right)$,

$$
f(x)+\lambda_{1} g(x)=b=f(x)+\lambda_{2} g(x) .
$$

Then $\lambda_{1}=\lambda_{2}$ follows as long as $g(x) \neq 0$. If we had $g(x)=0$, then the displayed equations would give $f(x)=b$, contradicting our assumption that no such $x$ exists.

The function $H$ given by the following proposition will serve as an envelope which controls the behavior at infinity of the members of the family of entire functions defined below.

Proposition 4.2. Let $\zeta: \mathbb{R} \rightarrow \mathbb{R}$ be given by $\zeta(x)=(1+|x|)^{-1}$. For any nondecreasing sequence $\left\{c_{i}\right\}_{i<\omega}$ of nonnegative real numbers with $\lim c_{i}=\infty$ and any positive continuous function $\varepsilon: \mathbb{R} \rightarrow \mathbb{R}$, there is an entire function $H$ such that

(a) $H[\mathbb{R}] \subseteq \mathbb{R}$,

(b) for all $x \in \mathbb{R}, H(x)>0$,

(c) for all $i<\omega$ and all $x \in \mathbb{R}$ such that $|x| \geq c_{i},\left|D^{i} H(x)\right|<2^{-i} \zeta(x) \varepsilon(x)$.

Remark 4.3. (1) Concerning the choice of $\zeta$, all that matters is that $\zeta$ is a continuous function such that $0<\zeta(x) \leq 1$ for all $x \in \mathbb{R}$ and $\lim _{x \rightarrow \pm \infty} \zeta(x)=0$. (2) It would be equivalent to state the proposition with $\varepsilon(x)$ instead of $2^{-i} \zeta(x) \varepsilon(x)$ in part (c), but the present formulation is more convenient for our purposes.

Proof. Choose a positive continuous function $\varepsilon^{\prime}$ such that for all $x \in \mathbb{R}$, if $|x| \geq c_{i}$, then $\varepsilon^{\prime}(x)<2^{-i} \zeta(x) \varepsilon(x)$. Consider functions of the form $H^{\prime}=\sum_{n=-\infty}^{\infty} \varepsilon_{n} g_{\left(\frac{n}{2}, \frac{n}{2}+1\right)}$, where $\varepsilon_{n}>0$ for each $n \in \mathbb{Z}$ and for each pair of real numbers $a<b, g_{(a, b)}$ is any $C^{\infty}$ function which is positive on $(a, b)$ and zero elsewhere. It is clear that for a suitable choice of the coefficients $\varepsilon_{n}$, we have that $H^{\prime}$ is a positive $C^{\infty}$ function satisfying $\left|D^{i} H^{\prime}(x)\right|<\frac{1}{2} \varepsilon^{\prime}(x)$ for all $i<\omega$ and all $x \in \mathbb{R}$ such that $|x| \geq c_{i}$. By Theorem 1.11, there is an entire function $H$ such that $H[\mathbb{R}] \subseteq \mathbb{R}, H(x)>0$ for all $x \in \mathbb{R}$, and for all $i<\omega$ and all $x \in \mathbb{R}$ such that $|x| \geq c_{i},\left|D^{i} H(x)-D^{i} H^{\prime}(x)\right|<\frac{1}{2} \varepsilon^{\prime}(x)$. Then, for all $i<\omega$ and all $x \in \mathbb{R}$ such that $|x| \geq c_{i},\left|D^{i} H(x)\right|<\varepsilon^{\prime}(x)$ and hence (c) holds.

Let $\mathcal{G}$ be the family of entire functions $g(n, A)$ where $n \in \mathbb{N}, A \subseteq \mathbb{R}$ is a nonvoid finite set, and for all $z \in \mathbb{C}$,

$$
g(n, A)(z)=\prod_{a \in A} \sin \left(\frac{z-a}{n}\right) .
$$

Let $\mathcal{G}_{0}$ be the subfamily consisting of those functions $g(n, A)$ for which $n \geq 4|A|$. The next proposition gathers some simple properties of the collection $\mathcal{G}$.

Proposition 4.4. The family $\mathcal{G}$ satisfies the following properties.

(a) For all $i<\omega$ and all $x \in \mathbb{R},\left|D^{i} g(n, A)(x)\right| \leq(|A| / n)^{i}$.

(b) Let $I_{a}, a \in A$, be pairwise disjoint open intervals such that $a \in I_{a}$, for each $a \in A$. For each $a \in A$, let $\left(r_{a, m}: m \in \mathbb{N}\right)$ be a sequence in $I_{a}$ such that for 
each $m \in \mathbb{N}$ we have $\left|a-r_{a, m}\right|<1 / m$. Let $A(m)=\left\{r_{a, m}: a \in A\right\}$. Then for all $i<\omega, m \in \mathbb{N}$ and $x \in \mathbb{R}$,

$$
\left|D^{i} g(n, A)(x)-D^{i} g(n, A(m))(x)\right| \leq m^{-1}(|A| / n)^{i+1}
$$

and for all $z \in \mathbb{C}, m \in \mathbb{N}$ and $\lambda, \mu \in \mathbb{R}$, if $M>0$ and $|z| \leq M$, then $|g(n, A(m))(z)| \leq T_{1}$ and

$$
|\lambda g(n, A)(z)-\mu g(n, A(m))(z)| \leq T_{2}(|\lambda-\mu|+|\mu| / m),
$$

where $T_{1}$ and $T_{2}$ are constants which depend only on $n, A$ and $M$.

(c) Let $\left\{c_{i}\right\}_{i<\omega}$ be a nondecreasing sequence of nonnegative real numbers with $\lim c_{i}=\infty$ and let $\varepsilon: \mathbb{R} \rightarrow \mathbb{R}$ be a positive continuous function. Let $H: \mathbb{C} \rightarrow \mathbb{C}$ be as given by Proposition 4.2 . Write $H$ span $\mathcal{G}_{0}$ for the set of functions of the form $H G$ where $G \in \operatorname{span} \mathcal{G}_{0}$ and $\operatorname{span} \mathcal{G}_{0}$ is the set of all real linear combinations of elements of $\mathcal{G}_{0}$. Then for all $i<\omega$, for all $f \in H \operatorname{span} \mathcal{G}_{0}$ and for all $x \in \mathbb{R}$ such that $|x| \geq c_{i}$, we have $\left|D^{i} f(x)\right| \leq\left(\sum_{g \in \mathcal{G}^{\prime}}\left|\lambda_{g}\right|\right)(3 / 4)^{i} \zeta(x) \varepsilon(x)$, where $f=H \sum\left\{\lambda_{g} g: g \in \mathcal{G}^{\prime}\right\}$ for some finite $\mathcal{G}^{\prime} \subseteq \mathcal{G}_{0}$ and $\lambda_{g} \in \mathbb{R}, g \in \mathcal{G}^{\prime}$. ( $\zeta$ is as in Proposition 4.2.)

Proof. (a) By induction on $i$, it follows that the $i$-th derivative of $g(n, A)$ can be expressed as a sum of $|A|^{i}$ terms, each of which is equal to $\pm 1 / n^{i}$ times a product indexed by $A$ in which the factor corresponding to $a \in A$ is equal to $\sin ((z-a) / n)$ or $\cos ((z-a) / n)$. The inequality in (a) follows since for $z \in \mathbb{R}$, the factors $\sin ((z-a) / n)$ and $\cos ((z-a) / n)$ are bounded in absolute value by 1 .

(b) Note that for any natural number $k$ and for any numbers $u_{i}, v_{i} \in \mathbb{C}$ of modulus at most $\mu_{0}, i=1, \ldots, k$, we have

$$
\begin{aligned}
& \left|\left(u_{1} u_{2} \ldots u_{k}\right)-\left(v_{1} v_{2} \ldots v_{k}\right)\right| \\
& =\left|\sum_{i=1}^{k}\left(v_{1} \ldots v_{i-1} u_{i} u_{i+1} \ldots u_{k}\right)-\left(v_{1} \ldots v_{i-1} v_{i} u_{i+1} \ldots u_{k}\right)\right| \\
& \leq \sum_{i=1}^{k}\left|v_{1} \ldots v_{i-1}\left(u_{i}-v_{i}\right) u_{i+1} \ldots u_{k}\right| \\
& \leq \sum_{i=1}^{k} \mu_{0}^{k-1}\left|u_{i}-v_{i}\right| .
\end{aligned}
$$

For $x \in \mathbb{R}$, the difference between $D^{i} g(n, A)(x)$ and $D^{i} g(n, A(m))(x)$ can be expressed, as in the argument for (a), as a sum of $|A|^{i}$ terms, each of which is equal to $\pm 1 / n^{i}$ times a difference of the form

$$
\prod_{a \in A} f_{a}\left(\frac{x-a}{n}\right)-\prod_{a \in A} f_{a}\left(\frac{x-r_{a, m}}{n}\right)
$$

where each $f_{a}$ is either a sine or a cosine. Applying (4.1) with $\mu_{0}=1$ to these differences and using that, by the Mean Value Theorem, for $x \in \mathbb{R}$ and $a \in A$ we have

$$
\left|f_{a}\left(\frac{x-a}{n}\right)-f_{a}\left(\frac{x-r_{a, m}}{n}\right)\right| \leq(1 / n)\left|r_{a, m}-a\right|
$$


we get that for each $x \in \mathbb{R}$,

$$
\left|D^{i} g(n, A)(x)-D^{i} g(n, A(m))(x)\right| \leq \frac{|A|^{i}}{n^{i}} \sum_{a \in A}(1 / n)\left|r_{a, m}-a\right| \leq \frac{|A|^{i+1}}{m n^{i+1}} .
$$

This takes care of the first part of (b). For the second, we have that if $|z| \leq M$, then $\left|z-r_{a, m}\right| \leq|z-a|+\left|a-r_{a, m}\right| \leq M+|a|+1$. Hence, letting $T_{1}$ denote the supremum of $\left|\prod_{a \in A} \sin \left(z_{a} / n\right)\right|$ over all choices of $z_{a} \in \mathbb{C}$ such that $\left|z_{a}\right| \leq M+|a|+1, a \in A$, we get $|g(n, A(m))(z)| \leq T_{1}$. For the remaining part of (b), we use

$$
\sin \left(\frac{z-a}{n}\right)-\sin \left(\frac{z-r_{a, m}}{n}\right)=2 \cos \left(\frac{2 z-r_{a, m}-a}{2 n}\right) \sin \left(\frac{r_{a, m}-a}{2 n}\right)
$$

and apply (4.1) to the difference $g(n, A)(z)-g(n, A(m))(z)$ with $\mu_{0}$ taken to be the supremum of 1 and all the quantities $|\sin ((z-r) / n)|$, $|\cos ((2 z-r-a) /(2 n))|$ and $|g(n, A)(z)|$, where $z, r, a$ range over the values given by $|z| \leq M, r \in \bigcup_{a \in A}[a-1, a+1], a \in A$. This gives that for $|z| \leq M$ and $m \in \mathbb{N}$ we have

$$
\begin{aligned}
|g(n, A)(z)-g(n, A(m))(z)| & \leq \mu_{0}^{|A|-1} \sum_{a \in A}\left|\sin \left(\frac{z-a}{n}\right)-\sin \left(\frac{z-r_{a, m}}{n}\right)\right| \\
& \leq 2 \mu_{0}^{|A|} \sum_{a \in A}\left|\sin \left(\frac{r_{a, m}-a}{2 n}\right)\right| .
\end{aligned}
$$

Since $\left|\sin \left(\left(r_{a, m}-a\right) /(2 n)\right)\right| \leq\left|\left(r_{a, m}-a\right) /(2 n)\right| \leq 1 / m$, we have

$$
\begin{aligned}
|\lambda g(n, A)(z)-\mu g(n, A(m))(z)| \leq & |\lambda-\mu||g(n, A)(z)| \\
& +|\mu||g(n, A)(z)-g(n, A(m))(z)| \\
\leq & \mu_{0}|\lambda-\mu|+2|A| \mu_{0}^{|A|}|\mu| / m \\
\leq & 2|A| \mu_{0}^{|A|}(|\lambda-\mu|+|\mu| / m) .
\end{aligned}
$$

(c) Let $f \in H \operatorname{span} \mathcal{G}_{0}$ and let $i<\omega$. Write

$$
f=H \sum\left\{\lambda_{s} s: s \in \mathcal{G}^{\prime}\right\}
$$

for some finite $\mathcal{G}^{\prime} \subseteq \mathcal{G}_{0}$ and $\lambda_{s} \in \mathbb{R}, s=g\left(n_{s}, A_{s}\right) \in \mathcal{G}^{\prime}$. We have

$$
D^{i} f=\sum\left\{\lambda_{s} D^{i}(H s): s \in \mathcal{G}^{\prime}\right\} .
$$

For each $s \in \mathcal{G}^{\prime}$ we have, using (a), the bound (for $x \in \mathbb{R}$ )

$$
\left|D^{i}(H s)(x)\right| \leq \sum_{k=0}^{i}\left(\begin{array}{l}
i \\
k
\end{array}\right)\left|D^{k} H(x)\right|\left|D^{i-k} s(x)\right| \leq \sum_{k=0}^{i}\left(\begin{array}{l}
i \\
k
\end{array}\right)\left|D^{k} H(x)\right|\left(\left|A_{s}\right| / n_{s}\right)^{i-k} .
$$

Using $n_{s} \geq 4\left|A_{s}\right|$, this gives

$$
\left|D^{i} f(x)\right| \leq \sum_{s \in \mathcal{G}^{\prime}}\left|\lambda_{s}\right| \sum_{k=0}^{i}\left(\begin{array}{l}
i \\
k
\end{array}\right)\left|D^{k} H(x)\right| \frac{1}{4^{i-k}} .
$$


When $|x| \geq c_{i}$, we have $|x| \geq c_{k}, 0 \leq k \leq i$ and so

$$
\left|D^{i} f(x)\right| \leq \sum_{s \in \mathcal{G}^{\prime}}\left|\lambda_{s}\right| \sum_{k=0}^{i}\left(\begin{array}{l}
i \\
k
\end{array}\right) \frac{1}{2^{k}} \frac{1}{4^{i-k}} \zeta(x) \varepsilon(x)=\left(\sum_{s \in \mathcal{G}^{\prime}}\left|\lambda_{s}\right|\right)(3 / 4)^{i} \zeta(x) \varepsilon(x) .
$$

Proposition 4.5. Let $N$ be an elementary submodel of $H_{\theta}$ for some regular $\theta>\mathfrak{c}$. Let $\left\{c_{i}\right\}_{i<\omega} \in N$ be a nondecreasing sequence of nonnegative real numbers with $\lim c_{i}=\infty$. Let $\varepsilon: \mathbb{R} \rightarrow \mathbb{R}$ be positive and continuous, $\varepsilon \in N$. Let $H: \mathbb{C} \rightarrow \mathbb{C}$ be as given by Proposition 4.2, $H \in N$. Let $M>0$. Let $B, C$ be countable dense subsets of $\mathbb{R}, B, C \in N$. Let $u \in \mathbb{R}$. Let $f_{0}$ be an entire function such that $f_{0}[\mathbb{R}] \subseteq \mathbb{R}$, $f_{0} \in N$. Let $f \in f_{0}+H$ span $\mathcal{G}_{0}$. Let $K_{0}, K_{1} \subseteq \mathbb{R}$ be finite such that $K_{0}, f\left[K_{1}\right] \in N$. Let $h \subseteq f$ be finite such that $h \in N$. Assume that the sets $\operatorname{dom} h, K_{0}, K_{1},\{u\}$ are pairwise disjoint. Then there is a function $f^{\prime} \in N \cap\left(f_{0}+H\right.$ span $\left.\mathcal{G}_{0}\right)$ such that for some $n \in \mathbb{N}$ and some positive rational number $r$, the following properties hold. In this list, $K$ denotes the set $K_{0} \cup\left\{b_{x}: x \in K_{1}\right\} \cup \operatorname{dom} h$ ( $b_{x}$ is defined in (c) below).

(a) $h \subseteq f^{\prime}$.

(b) For all $x \in K_{0}, f^{\prime}(x) \in C$.

(c) For all $x \in K_{1}$, there is a $b_{x} \in B$ such that $\left|b_{x}-x\right|<\varepsilon(0)$ and $f^{\prime}\left(b_{x}\right)=f(x)$.

(d) $n \geq 4|K|$ and for all $i<\omega$ and for all $\sigma \in \mathbb{R}$ such that $|\sigma| \leq r$, we have that for $x \in \mathbb{R}$ such that $|x| \geq c_{i}$,

$$
\left|D^{i} f(x)-D^{i}\left(f^{\prime}+\sigma H g(n, K)\right)(x)\right|<\varepsilon(x) .
$$

(e) $g(n, K)(u) \neq 0$ and for some real number $\sigma$ such that $|\sigma|<r$, we have

$$
f(u)=f^{\prime}(u)+\sigma H(u) g(n, K)(u) .
$$

(f) For all $z \in \mathbb{C}$ such that $|z| \leq M$ and for all $\sigma \in \mathbb{R}$ such that $|\sigma| \leq r$,

$$
r|H(z) g(n, K)(z)|<\varepsilon(0) \text { and }\left|f(z)-\left(f^{\prime}+\sigma H g(n, K)\right)(z)\right|<\varepsilon(0) .
$$

Remark 4.6. Under the hypotheses of the proposition, we shall have occasion to want the conclusion for $\lambda \varepsilon$ instead of $\varepsilon$, where $\lambda$ is a rational number such that $0<\lambda<1$. To see that this modified conclusion holds, we argue as follows. First notice that it follows from properties (a), (b) and (c) of Proposition 4.2 that the same properties hold for $(\lambda \varepsilon, \lambda H)$ instead of $(\varepsilon, H)$. Since $\lambda$ is rational, $\lambda \varepsilon, \lambda H \in N$. Since $(\lambda H)$ span $\mathcal{G}_{0}=H \operatorname{span} \mathcal{G}_{0}$, we have $f \in f_{0}+(\lambda H)$ span $\mathcal{G}_{0}$. The proposition therefore gives $f^{\prime} \in f_{0}+(\lambda H) \operatorname{span} \mathcal{G}_{0}=f_{0}+H \operatorname{span} \mathcal{G}_{0}, f^{\prime} \in N, n \in \mathbb{N}$ and a positive rational number $r$ such that (a)-(f) hold with $\lambda \varepsilon$ and $\lambda H$ in the place of $\varepsilon$ and $H$, respectively. But now notice that everywhere $H$ is mentioned, i.e., in (d), (e) and (f), it is multiplied by $r$ or by some $\sigma$ such that $|\sigma| \leq r$. Equivalent statements of these clauses are obtained by restoring $\lambda H$ to $H$ and replacing $r$ by $\lambda r$, giving the desired modification of the conclusion.

Proof. Choose pairwise disjoint open intervals $I_{x}$ such that $x \in I_{x}$ and $I_{x} \cap(\{u\} \cup$ $\left.K_{0} \cup \operatorname{dom} h\right)=\emptyset$, for $x \in K_{1}$. For each $x \in K_{1}$, choose a sequence of points $b_{x, m} \in B \cap I_{x}, m \in \mathbb{N}$, such that $\left|b_{x, m}-x\right|<1 / m$. Let

$$
\begin{gathered}
\bar{K}=K_{0} \cup K_{1} \cup \operatorname{dom} h, \\
\bar{K}_{m}=K_{0} \cup\left\{b_{x, m}: x \in K_{1}\right\} \cup \operatorname{dom} h .
\end{gathered}
$$


For each $x \in K_{0}$, choose a sequence of points $c_{x, m} \in C, m \in \mathbb{N}$, such that $\left|c_{x, m}-f(x)\right|<1 / m$. Let

$$
f=f_{0}+H \sum\left\{\lambda_{s} s: s \in \mathcal{G}^{\prime}\right\}
$$

where $\mathcal{G}^{\prime}$ is a finite subset of $\mathcal{G}_{0}$ and $\lambda_{s} \in \mathbb{R}$ for each $s \in \mathcal{G}^{\prime}$. For each $s \in \mathcal{G}^{\prime}$, let $n(s) \in \mathbb{N}$ and $A(s) \subseteq \mathbb{R}$ be such that $s=g(n(s), A(s))$. For each $s \in \mathcal{G}^{\prime}$, pick pairwise disjoint open intervals $I_{a}^{s}$ such that $a \in I_{a}^{s}$, for $a \in A(s)$. For each $a \in A(s)$, choose a sequence of rational numbers $r_{a, m}^{s} \in I_{a}^{s}, m \in \mathbb{N}$, such that for each $m \in \mathbb{N}$ we have $\left|a-r_{a, m}^{s}\right|<1 / m$. Let $A(s, m)=\left\{r_{a, m}^{s}: a \in A(s)\right\}$. Choose $n \in \mathbb{N}$ large enough so that

(i) $u$ is not a zero of $g(n, \bar{K})$ or any $g\left(n, \bar{K}_{m}\right), m \in \mathbb{N}$,

(ii) for each $a \in \bar{K}_{m}, a$ is not a zero of $g\left(n, \bar{K}_{m} \backslash\{a\}\right)$,

(iii) for each $x \in \bar{K}, x$ is not a zero of $g(n, \bar{K} \backslash\{x\})$,

(iv) $n \geq 4\left|\bar{K}_{m}\right|=4\left(\left|K_{0}\right|+\left|K_{1}\right|+|\operatorname{dom} h|\right)$.

(This is possible because the sets $\left\{b_{x, m}: m \in \mathbb{N}\right\}$ are bounded so that for large enough $n$ we have $\left|a-b_{x, m}\right|,\left|u-b_{x, m}\right|<n \pi\left(a \in \bar{K}_{m}, x, \in K_{1}\right)$ for all $m$ simultaneously.) Consider functions $f^{\prime}, \bar{f}$ of the form

$$
f^{\prime}=f_{0}+H \sum\left\{\mu_{s} g(n(s), A(s, m)): s \in \mathcal{G}^{\prime}\right\}+H \sum\left\{\sigma_{a} g\left(n, \bar{K}_{m} \backslash\{a\}\right): a \in \bar{K}_{m}\right\}
$$

and

$$
\begin{aligned}
\bar{f}= & f_{0}+H \sum\left\{\mu_{s} g(n(s), A(s, m)): s \in \mathcal{G}^{\prime}\right\}+H \sum\left\{\sigma_{a} g\left(n, \bar{K}_{m} \backslash\{a\}\right): a \in \bar{K}_{m}\right\} \\
& +\sigma H g\left(n, \bar{K}_{m}\right) \\
= & f^{\prime}+\sigma H g\left(n, \bar{K}_{m}\right)
\end{aligned}
$$

where $m \in \mathbb{N}, \mu_{s}, \sigma_{a}, \sigma \in \mathbb{R}\left(s \in \mathcal{G}^{\prime}, a \in \bar{K}_{m}\right)$. For $a \in \bar{K}_{m}$, among the functions $g\left(n, \bar{K}_{m} \backslash\left\{a^{\prime}\right\}\right), a^{\prime} \in \bar{K}_{m}$, only the one with $a^{\prime}=a$ is not zero at $a$. This leads to the following observations.

(1) For $a \in \operatorname{dom} h$ and for each choice of $m \in \mathbb{N}$ and $\vec{\mu}=\left(\mu_{s}\right)_{s \in \mathcal{G}^{\prime}}$, there is a unique $\sigma_{a}=\sigma_{a}(m, \vec{\mu})$ for which $f^{\prime}(a)=f(a)=h(a)$, namely

$$
\sigma_{a}(m, \vec{\mu})=\frac{\sum\left\{\lambda_{s} g(n(s), A(s))(a)-\mu_{s} g(n(s), A(s, m))(a): s \in \mathcal{G}^{\prime}\right\}}{g\left(n, \bar{K}_{m} \backslash\{a\}\right)(a)} .
$$

(2) For each $x \in K_{0}$ and each choice of $m \in \mathbb{N}$ and $\vec{\mu}=\left(\mu_{s}\right)_{s \in \mathcal{G}^{\prime}}$, there is a unique $\sigma_{x}=\sigma_{x}(m, \vec{\mu})$ for which $f^{\prime}(x)=c_{x, m}$, namely

$$
\begin{aligned}
& \sigma_{x}(m, \vec{\mu})=\frac{c_{x, m}-f_{0}(x)-H(x) \sum\left\{\mu_{s} g(n(s), A(s, m))(x): s \in \mathcal{G}^{\prime}\right\}}{H(x) g\left(n, \bar{K}_{m} \backslash\{x\}\right)(x)} \\
& =\frac{c_{x, m}-f(x)+H(x) \sum\left\{\lambda_{s} g(n(s), A(s))(x)-\mu_{s} g(n(s), A(s, m))(x): s \in \mathcal{G}^{\prime}\right\}}{H(x) g\left(n, \bar{K}_{m} \backslash\{x\}\right)(x)} .
\end{aligned}
$$


(3) For each $x \in K_{1}$ and each choice of $m \in \mathbb{N}$ and $\vec{\mu}=\left(\mu_{s}\right)_{s \in \mathcal{G}^{\prime}}$, there is a unique $\sigma_{b_{x, m}}=\sigma_{b_{x, m}}(m, \vec{\mu})$ for which $f^{\prime}\left(b_{x, m}\right)=f(x)$, namely

$$
\begin{gathered}
\sigma_{b_{x, m}}(m, \vec{\mu})=\frac{f(x)-f_{0}\left(b_{x, m}\right)-H\left(b_{x, m}\right) \sum_{s \in \mathcal{G}^{\prime}} \mu_{s} g(n(s), A(s, m))\left(b_{x, m}\right)}{H\left(b_{x, m}\right) g\left(n, \bar{K}_{m} \backslash\left\{b_{x, m}\right\}\right)\left(b_{x, m}\right)} \\
f_{0}(x)-f_{0}\left(b_{x, m}\right)+H(x) \sum_{s \in \mathcal{G}^{\prime}} \lambda_{s} g(n(s), A(s))(x) \\
=\frac{-H\left(b_{x, m}\right) \sum_{s \in \mathcal{G}^{\prime}} \mu_{s} g(n(s), A(s, m))\left(b_{x, m}\right)}{H\left(b_{x, m}\right) g\left(n, \bar{K}_{m} \backslash\left\{b_{x, m}\right\}\right)\left(b_{x, m}\right)} \\
f_{0}(x)-f_{0}\left(b_{x, m}\right)+\left[H(x)-H\left(b_{x, m}\right)\right] \sum_{s \in \mathcal{G}^{\prime}} \lambda_{s} g(n(s), A(s))(x) \\
+H\left(b_{x, m}\right) \sum_{s \in \mathcal{G}^{\prime}} \lambda_{s}\left(g(n(s), A(s))(x)-g(n(s), A(s))\left(b_{x, m}\right)\right) \\
+H\left(b_{x, m}\right) \sum_{s \in \mathcal{G}^{\prime}}\left(\lambda_{s} g(n(s), A(s))\left(b_{x, m}\right)-\mu_{s} g(n(s), A(s, m))\left(b_{x, m}\right)\right) \\
H\left(b_{x, m}\right) g\left(n, \bar{K}_{m} \backslash\left\{b_{x, m}\right\}\right)\left(b_{x, m}\right)
\end{gathered} .
$$

(4) Given the assignment of values $\sigma_{a}=\sigma_{a}(m, \vec{\mu}), a \in \bar{K}_{m}$, there is a unique value of $\sigma=\sigma(m, \vec{\mu})$ for which $\bar{f}(u)=f(u)$, namely

$$
\begin{aligned}
& \sum_{s \in \mathcal{G}^{\prime}}\left[\lambda_{s} g(n(s), A(s))(u)-\mu_{s} g(n(s), A(s, m))(u)\right] \\
& -\sum_{a \in \bar{K}_{m}} \sigma_{a}(m, \vec{\mu}) g\left(n, \bar{K}_{m} \backslash\{a\}\right)(u) \\
& \sigma(m, \vec{\mu})=\frac{a \in \bar{K}_{m}}{g\left(n, \bar{K}_{m}\right)(u)}
\end{aligned}
$$

Note that for functions $f^{\prime}$, if $\sigma_{a}=\sigma_{a}(m, \vec{\mu})$ for each $a \in \bar{K}_{m}$, then (a), (b) hold and (c) holds (with $b_{x}=b_{x, m}$ ) if $m$ is large enough. Also, as $\vec{\mu}=\left(\mu_{s}\right)_{s \in \mathcal{G}^{\prime}} \rightarrow\left(\lambda_{s}\right)_{s \in \mathcal{G}^{\prime}}$ and $m \rightarrow \infty$, we have $\sigma_{a}(m, \vec{\mu}) \rightarrow 0$ for each $a \in K_{0} \cup \operatorname{dom} h, \sigma_{b_{x, m}}(m, \vec{\mu}) \rightarrow 0$ for each $x \in K_{1}$, and then it follows that $\sigma(m, \vec{\mu}) \rightarrow 0$ as well. To check this, use the consequences of Proposition 4.4 (part (a) and the first part of (b), both with $i=0$ ) that whenever $n^{\prime} \geq\left|A^{\prime}\right|$, we have for all $x \in \mathbb{R},\left|g\left(n^{\prime}, A^{\prime}\right)(x)\right| \leq$ 1 and $\left|g\left(n^{\prime}, A^{\prime}(m)\right)(x)-g\left(n^{\prime}, A^{\prime}\right)(x)\right| \leq 1 / m$. In the present circumstances, we have $\bar{K}$ and $\bar{K}_{m}$ (as well as the pairs $\left(\bar{K} \backslash\{a\}, \bar{K}_{m} \backslash\{a\}\right), a \in K_{0} \cup \operatorname{dom} h$, and $\left.\left(\bar{K} \backslash\{x\}, \bar{K}_{m} \backslash\left\{b_{x, m}\right\}\right), x \in K_{1}\right)$ playing the role of $A^{\prime}$ and $A^{\prime}(m)$. Note in particular 
that the denominators in (1)-(4) are bounded away from zero. For example, for the denominator in (3) we have that $H\left(b_{x, m}\right) \rightarrow H(x)>0$ and, by the choice of $n$, $g(n, \bar{K} \backslash\{x\})(x) \neq 0$ and

$$
\begin{aligned}
\mid g\left(n, \bar{K}_{m} \backslash\left\{b_{x, m}\right\}\right)( & \left.b_{x, m}\right)-g(n, \bar{K} \backslash\{x\})(x) \mid \\
\leq & \left|g\left(n, \bar{K}_{m} \backslash\left\{b_{x, m}\right\}\right)\left(b_{x, m}\right)-g(n, \bar{K} \backslash\{x\})\left(b_{x, m}\right)\right| \\
& +\left|g(n, \bar{K} \backslash\{x\})\left(b_{x, m}\right)-g(n, \bar{K} \backslash\{x\})(x)\right| \\
\leq & 1 / m+\left|g(n, \bar{K} \backslash\{x\})\left(b_{x, m}\right)-g(n, \bar{K} \backslash\{x\})(x)\right| \\
& \rightarrow 0 \text { as } m \rightarrow \infty .
\end{aligned}
$$

For the rest of the proof, we restrict our attention to choices of the coefficients in the definitions of $f^{\prime}$ and $\bar{f}$ such that $\left|\mu_{s}-\lambda_{s}\right| \leq 1, s \in \mathcal{G}^{\prime},\left|\sigma_{a}\right| \leq 1, a \in \bar{K}_{m}$, and $|\sigma| \leq 1$. Given these restrictions, let $i_{0}<\omega$ be such that for all $i>i_{0}$, for all $m \in \mathbb{N}$, $\vec{\mu}=\left(\mu_{s}\right)_{s \in \mathcal{G}^{\prime}}$ and $x \in \mathbb{R}$, we have that if $|x| \geq c_{i}$, then $\left|D^{i}(\bar{f}-f)(x)\right|<\varepsilon(x)$. (Such an $i_{0}$ exists by Proposition 4.4 (c) which, because $\zeta(x) \leq 1$, yields $\left|D^{i}(\bar{f}-f)(x)\right|<$ $C(3 / 4)^{i} \varepsilon(x)$ whenever $|x| \geq c_{i}$, where $C$ is a constant independent of the choice of the coefficients in the definition $\bar{f}$ as long as the restrictions we just placed on these coefficients are respected.)

Now let $L$ be a compact interval such that for all $i \leq i_{0}$, for all $m \in \mathbb{N}$, for all $\vec{\mu}=\left(\mu_{s}\right)_{s \in \mathcal{G}^{\prime}}$ and for all $x \in \mathbb{R} \backslash L$ (and given the above restrictions on the choice of coefficients for $\bar{f}$ ), we have that if $|x| \geq c_{i}$, then $\left|D^{i}(\bar{f}-f)(x)\right|<\varepsilon(x)$. (Such an $L$ exists by Proposition 4.4(c) again. This time we use the fact that for each $i<\omega$ and $x$ such that $|x| \geq c_{i},\left|D^{i}(\bar{f}-f)(x)\right|<C(3 / 4)^{i} \zeta(x) \varepsilon(x) \leq C \zeta(x) \varepsilon(x)<\varepsilon(x)$ for $|x|$ large enough since $\lim _{x \rightarrow \pm \infty} \zeta(x)=0$.)

Henceforth, we limit ourselves to functions $f^{\prime}, \bar{f}$ for which, in addition to the restrictions imposed above, we have $\sigma_{a}=\sigma_{a}(m, \vec{\mu}), a \in \bar{K}_{m}$. (So we now consider only coefficients so that $|\sigma| \leq 1$ and for $a \in \bar{K}_{m}, \sigma_{a}=\sigma_{a}(m, \vec{\mu})$ with $\vec{\mu}$ close enough to $\vec{\lambda}$ and $m$ large enough so that the conditions $\left|\mu_{s}-\lambda_{s}\right| \leq 1, s \in \mathcal{G}^{\prime}$ and $\left|\sigma_{a}\right| \leq 1$, $a \in \bar{K}_{m}$ are satisfied.) Consider the following facts.

(5) For $|x| \geq c_{i},\left|D^{i} f(x)-D^{i} \bar{f}(x)\right|<\varepsilon(x)$ can only fail if $i \leq i_{0}$ and $x \in L$.

(6) For some $\delta>0$, we have $\varepsilon(x)>\delta$ for all $x \in L$.

(7) For $|z| \leq M$, we have

$$
\begin{aligned}
& |f(z)-\bar{f}(z)| \\
& \leq|H(z)|\left[\sum\left\{\left|\lambda_{s} g(n(s), A(s))(z)-\mu_{s} g(n(s), A(s, m))(z)\right|: s \in \mathcal{G}^{\prime}\right\}\right. \\
& \left.\quad+\sum\left\{\left|\sigma_{a}(m, \vec{\mu})\right|\left|g\left(n, \bar{K}_{m} \backslash\{a\}\right)(z)\right|: a \in \bar{K}_{m}\right\}+|\sigma|\left|g\left(n, \bar{K}_{m}\right)(z)\right|\right] \\
& \leq\left(\sup _{|z| \leq M}|H(z)|\right)\left[\sum\left\{T_{s, M}\left(\left|\lambda_{s}-\mu_{s}\right|+\left|\mu_{s}\right| / m\right): s \in \mathcal{G}^{\prime}\right\}\right. \\
& \left.\quad+\sum\left\{\left|\sigma_{a}(m, \vec{\mu})\right| T_{n, M}: a \in \bar{K}_{m}\right\}+|\sigma| T_{n, M}\right],
\end{aligned}
$$

where $T_{n, M}, T_{s, M}$ are constants independent of $m$ as in Proposition 4.4(b). 
(8) For $i \leq i_{0}$ and $x \in L$, we have

$$
\begin{aligned}
& \left|D^{i} f(x)-D^{i} \bar{f}(x)\right| \\
& \leq \sum_{k=0}^{i}\left(\begin{array}{l}
i \\
k
\end{array}\right)\left|D^{i-k} H(x)\right|\left[\sum_{s \in \mathcal{G}^{\prime}} \mid \lambda_{s} D^{k} g(n(s), A(s))(x)\right. \\
& -\mu_{s} D^{k} g(n(s), A(s, m))(x) \mid \\
& \left.+\sum_{a \in \bar{K}_{m}}\left|\sigma_{a}(m, \vec{\mu})\right|\left|D^{k} g\left(n, \bar{K}_{m} \backslash\{a\}\right)(x)\right|+|\sigma|\left|D^{k} g\left(n, \bar{K}_{m}\right)(x)\right|\right] \\
& \leq \sum_{k=0}^{i}\left(\begin{array}{l}
i \\
k
\end{array}\right)\left|D^{i-k} H(x)\right|\left[\sum_{s \in \mathcal{G}^{\prime}}\left(\left|\lambda_{s}-\mu_{s}\right|\left|D^{k} g(n(s), A(s))(x)\right|+\left|\mu_{s}\right| / m\right)\right. \\
& \left.+\sum_{a \in \bar{K}_{m}}\left|\sigma_{a}(m, \vec{\mu})\right|\left|D^{k} g\left(n, \bar{K}_{m} \backslash\{a\}\right)(x)\right|+|\sigma|\left|D^{k} g\left(n, \bar{K}_{m}\right)(x)\right|\right] \\
& \leq \sum_{k=0}^{i}\left(\begin{array}{l}
i \\
k
\end{array}\right) C_{H}\left[\sum_{s \in \mathcal{G}^{\prime}}\left(\left|\lambda_{s}-\mu_{s}\right|+\left|\mu_{s}\right| / m\right)+\sum_{a \in \bar{K}_{m}}\left|\sigma_{a}(m, \vec{\mu})\right|+|\sigma|\right],
\end{aligned}
$$

where $C_{H}=\sup _{x \in L, i \leq i_{0}}\left|D^{i} H(x)\right|$ and the second and third inequalities used the first part of Proposition $4.4(\mathrm{~b})$, the fact that $n(s) \geq|A(s)|$, Proposition 4.4(a) and the fact that $n \geq\left|\bar{K}_{m}\right|$.

We may choose $m \in \mathbb{N}, \mu_{s} \in \mathbb{Q}$ for each $s \in \mathcal{G}^{\prime}$ and $r>0$ so that (d), (e) and (f) are satisfied with $b_{x, m}$ in the place of $b_{x}$. (First get a neighborhood of $\vec{\lambda}$, an $m_{0}$ and an $r$ so that (d), (f) are satisfied for $\vec{\mu}$ in the given neighborhood of $\vec{\lambda}$ and $m \geq m_{0}$. Then choose such $\vec{\mu}$ and $m$ so that $|\sigma(m, \vec{\mu})|<r$, giving (e).) Note that we have $f^{\prime} \in N$ because $f_{0}, h, H, K_{0}, f\left[K_{1}\right],\left\{b_{x, m}: x \in K_{1}\right\},\left\{c_{x, m}: x \in K_{0}\right\} \in N$, each $\mu_{s}$ is rational (and hence belongs to $N$ ) and each $\sigma_{a}(m, \vec{\mu})$, for $a \in \bar{K}_{m}$, is uniquely determined by the condition that $f^{\prime}(a)=h(a)$ for $a \in \operatorname{dom} h, f^{\prime}(x)=c_{x, m}$ for $a=x \in K_{0}$ and $f^{\prime}\left(b_{x, m}\right)=f(x)$ for $a=b_{x, m}\left(x \in K_{1}\right)$, and hence belongs to $N$ by elementarity.

\section{MAIN LEMMA}

The next result is the main technical lemma for the oracle-cc iteration.

Lemma 5.1. Let $\bar{M}=\left\langle M_{\delta}: \delta<\omega_{1}\right\rangle$ be an oracle. Let $\left\langle A_{\alpha}: \alpha<\omega_{1}\right\rangle$ and $\left\langle B_{\alpha}: \alpha<\omega_{1}\right\rangle$ each be a sequence of pairwise disjoint subsets of $\mathbb{R}$ such that for $\alpha<\omega, A_{\alpha}$ and $B_{\alpha}$ are countable dense sets and for $\omega \leq \alpha<\omega_{1}, A_{\alpha}$ and $B_{\alpha}$ are everywhere second category sets of cardinality $\aleph_{1}$. Let $\left\{c_{i}\right\}_{i<\omega}$ be a nondecreasing sequence of nonnegative real numbers with $\lim c_{i}=\infty$. Let $\eta: \mathbb{R} \rightarrow \mathbb{R}$ be a positive continuous function. Let $f_{0}: \mathbb{C} \rightarrow \mathbb{C}$ be an entire function such that $f_{0}[\mathbb{R}] \subseteq \mathbb{R}$, $f_{0}\left\lceil\mathbb{R}\right.$ is an order-isomorphism and $D f_{0}(x)>0$ for all $x \in \mathbb{R}$. Then there is a forcing notion $P$ satisfying the $\bar{M}$-cc such that for every $G \subseteq P$ generic over $V$, 
$V[G] \models$ there is an entire function $f: \mathbb{C} \rightarrow \mathbb{C}$ for which the following properties hold:

(i) $f \uparrow \mathbb{R}$ is an order-isomorphism of $\mathbb{R}$,

(ii) $f\left[A_{\alpha}\right]=B_{\alpha}$ for all $\alpha<\omega_{1}$,

(iii) for all $i<\omega$ and all $x \in \mathbb{R}$ such that $|x| \geq c_{i},\left|D^{i} f(x)-D^{i} f_{0}(x)\right| \leq \eta(x)$.

Proof. By replacing $\eta(x)$ by $\min \left\{1, \eta(x), D f_{0}(x)\right\}$, we may assume that for all $x \in$ $\mathbb{R}, \eta(x) \leq 1$ and $\eta(x) \leq D f_{0}(x)$. We may also assume that $c_{0}=c_{1}=0$.

Let $H$ be as in Proposition 4.2 for $\left\{c_{i}\right\}_{i<\omega}$ and $\eta$. For the rest of the proof, fix a suitably large regular cardinal $\theta$. Let $\left\langle I_{n}: n<\omega\right\rangle$ list all the nonempty open intervals with rational endpoints. Fix a well-ordering of $\mathbb{R}$ in type $\omega_{1}$. (CH holds because there is an oracle.) Let $Q$ denote the set $\omega_{1} \times \omega \times 2$ equipped with the lexicographical order, which we denote by $\triangleleft$. We will inductively define partial orders $P(u), u \in Q$, from the following class of partial orders.

Definition 5.2. Let $N \prec H_{\theta}$. Let $\bar{a}=\left\langle a_{\xi}: \xi<\alpha\right\rangle, \bar{b}=\left\langle b_{\xi}: \xi<\beta\right\rangle$ be one-to-one sequences of real numbers, $\alpha, \beta \leq \omega_{1}$. We write, for $\delta<\omega_{1}$,

$$
\bar{a}^{\delta}=\left\{a_{\omega \delta+n}: n<\omega, \omega \delta+n<\alpha\right\} \quad \text { and } \quad \bar{b}^{\delta}=\left\{b_{\omega \delta+n}: n<\omega, \omega \delta+n<\beta\right\} .
$$

$P(\bar{a}, \bar{b}, N)$ denotes the partial order consisting of conditions $p=\left(h_{p}, f_{p}, \varepsilon_{p}, n_{p}\right)$ such that

(i) $h_{p}$ is a finite partial order-preserving map from $\left\{a_{\xi}: \xi<\alpha\right\}$ to $\left\{b_{\xi}: \xi<\beta\right\}$,

(ii) $h_{p}\left[\bar{a}^{\delta}\right] \subseteq \bar{b}^{\delta}$ for all $\delta<\omega_{1}$,

(iii) $f_{p} \in\left(f_{0}+H \operatorname{span} \mathcal{G}_{0}\right) \cap N$,

(iv) $h_{p} \subseteq f_{p}$,

(v) for all $i<\omega$ and all $x \in \mathbb{R}$ such that $|x| \geq c_{i},\left|D^{i}\left(f_{p}-f_{0}\right)(x)\right|<\left(1-\varepsilon_{p}\right) \eta(x)$,

(vi) $\varepsilon_{p}$ is a rational number, $0<\varepsilon_{p}<1$, and $n_{p}<\omega$.

The order is given by $p \leq q$ if and only if

(vii) $h_{p} \supseteq h_{q}, n_{p} \geq n_{q}$,

(viii) for all $z \in \mathbb{C}$ such that $|z| \leq n_{q},\left|f_{p}(z)-f_{q}(z)\right|+\varepsilon_{p} \leq \varepsilon_{q}$.

(This gives in particular $\varepsilon_{p} \leq \varepsilon_{q}$.)

This order relation is transitive because if $p \leq q \leq r$, then $h_{p} \supseteq h_{q} \supseteq h_{r}$, $n_{p} \geq n_{q} \geq n_{r}$ and for $z \in \mathbb{C}$ such that $|z| \leq n_{r}$, we have $|z| \leq n_{q}$ as well and hence

$$
\begin{aligned}
\left|f_{p}(z)-f_{r}(z)\right|+\varepsilon_{p} & \leq\left|f_{q}(z)-f_{r}(z)\right|+\left(\left|f_{p}(z)-f_{q}(z)\right|+\varepsilon_{p}\right) \\
& \leq\left|f_{q}(z)-f_{r}(z)\right|+\varepsilon_{q} \\
& \leq \varepsilon_{r} .
\end{aligned}
$$

Remark 5.3. The clauses of the definition together with the assumptions at the beginning of the proof ensure that $f_{p}\lceil\mathbb{R}$ is an order-isomorphism. We are assuming $c_{1}=0$ so that by (v), for all $x \in \mathbb{R},\left|D f_{p}(x)-D f_{0}(x)\right|<\left(1-\varepsilon_{p}\right) \eta(x)$. Thus,

$$
D f_{p}(x)>D f_{0}(x)-\left(1-\varepsilon_{p}\right) \eta(x) \geq \eta(x)-\left(1-\varepsilon_{p}\right) \eta(x)=\varepsilon_{p} \eta(x)>0 .
$$

Hence, $f_{p}$ is increasing. Because $c_{0}=0$ we also have, for all $x \in \mathbb{R},\left|f_{p}(x)-f_{0}(x)\right|<$ $\left(1-\varepsilon_{p}\right) \eta(x)<\eta(x) \leq 1$. Because $f_{0}$ is onto, it follows that $f_{p}$ is as well.

Fix a function $\gamma: \omega_{1} \rightarrow\left(\omega_{1} \backslash \omega\right) \times 2$ so that for each $(\alpha, i) \in\left(\omega_{1} \backslash \omega\right) \times 2$, $\left|\gamma^{-1}(\alpha, i)\right|=\aleph_{1}$.

To each $u=(\delta, n, j) \in Q$ we associate a pair of ordinals $(\alpha, \beta)=(\alpha(u), \beta(u))$ as follows. Let $\gamma(\delta)=\left(\alpha^{\prime}, i\right)$. If $j=0$, let $(\alpha, \beta)$ be the pair of ordinals $(\omega \delta+n, \omega \delta+n)$. 
If $j=1$, let $(\alpha, \beta)$ be the pair $(\omega \delta+n+1, \omega \delta+n)$ if $i=0$, and $(\omega \delta+n, \omega \delta+n+1)$ if $i=1$.

(Notice that the pair $(\alpha(u), \beta(u))$ uniquely determines $u$ : from $(\alpha, \beta)$ we can clearly recover $\delta$ and $n$, and we have $j=0$ if $\alpha=\beta$ and $j=1$ otherwise.)

We inductively define one-to-one enumerations $\bar{a}=\left\langle a_{\xi}: \xi<\omega_{1}\right\rangle$ of $\bigcup_{\alpha<\omega_{1}} A_{\alpha}$ and $\bar{b}=\left\langle b_{\xi}: \xi<\omega_{1}\right\rangle$ of $\bigcup_{\alpha<\omega_{1}} B_{\alpha}$ and a continuous $\in$-increasing sequence $\left\langle N_{u}\right.$ : $(\omega, 0,0) \unlhd u \in Q\rangle$ of countable elementary submodels of $H_{\theta}$, and then for each $u \in Q$ such that $(\omega, 0,0) \unlhd u$, we define

$$
P(u)=P\left(\overline { a } \left\lceil\alpha(u), \bar{b}\left\lceil\beta(u), N_{u}\right) .\right.\right.
$$

We simplify the notation by writing $P(u)=P(\bar{a} \uparrow \alpha(u), \bar{b} \uparrow \beta(u))$, omitting the explicit mention of $N_{u}$. No confusion should arise since $\alpha(u)$ and $\beta(u)$ uniquely determine $u$, and hence $N_{u}$. The indexing of the induction is such that for fixed $\delta<\omega_{1}$ such that $\gamma(\delta)=(\alpha, i)$, at stage $u=(\delta, n, j)$ of the induction, if $i=0$, then we pick $a_{\omega \delta+n} \in A_{\alpha}$ if $j=0$ and $b_{\omega \delta+n} \in B_{\alpha}$ if $j=1$, whereas if $i=1$, then we pick $b_{\omega \delta+n} \in B_{\alpha}$ if $j=0$ and $a_{\omega \delta+n} \in A_{\alpha}$ if $j=1$. In other words, the elements of $\bar{a}^{\delta}$ and $\bar{b}^{\delta}$ are chosen alternately from $A_{\alpha}$ and $B_{\alpha}$, respectively, starting with an element of $A_{\alpha}$ when $i=0$ and with an element of $B_{\alpha}$ when $i=1$. For technical reasons, we also define a second sequence $\left\langle N_{u}^{\prime}:(\omega, 0,0) \unlhd u \in Q\right\rangle$ of countable elementary submodels of $H_{\theta}$ and functions $e_{\delta}, \omega \leq \delta<\omega_{1}$.

We will arrange that the following conditions hold for all $u=(\delta, n, j) \in Q$, with $\gamma(\delta)=(\alpha, i)$

(1) For $\delta<\omega$, the only requirements are that $\bar{a}^{\delta}=A_{\delta}$ and $\bar{b}^{\delta}=B_{\delta}$, where $A_{\delta}$ and $B_{\delta}$ are the countable dense sets from the hypothesis.

(2) For $\delta \geq \omega, N_{u} \in N_{u}^{\prime}$ are countable elementary submodels of $H_{\theta}$.

(3) $f_{0},\left\langle c_{i}: i<\omega\right\rangle, \eta, H,\left\langle A_{\alpha}: \alpha<\omega_{1}\right\rangle,\left\langle B_{\alpha}: \alpha<\omega_{1}\right\rangle$ are all elements of $N_{(\omega, 0,0)}$.

(4) If $\delta \geq \omega$ and $(n, j)=(0,0)$, then

(i) $N_{u}=\bigcup\left\{N_{v}:(\omega, 0,0) \unlhd v \triangleleft u\right\}$;

(ii) if $i=0$, then $a_{\omega \delta}$ is the least element of $A_{\alpha} \backslash\left\{a_{\xi}: \xi<\omega \delta\right\}$;

(iii) if $i=1$, then $b_{\omega \delta}$ is the least element of $B_{\alpha} \backslash\left\{b_{\xi}: \xi<\omega \delta\right\}$.

In (ii) and (iii), "least" refers to the well-ordering of $\mathbb{R}$ fixed earlier.

(5) If $\delta \geq \omega$ and $(n, j) \neq(0,0)$, then

(i) $e_{\delta},\left\langle a_{\xi}: \xi<\alpha(u)\right\rangle,\left\langle b_{\xi}: \xi<\beta(u)\right\rangle,\left\langle M_{\delta^{\prime}}: \delta^{\prime} \leq \delta\right\rangle$, and $\left\langle N_{v}: v \triangleleft u\right\rangle$ all belong to $N_{u}$.

(ii) If $i=0, j=0$ or $i=1, j=1$, then $a_{\omega \delta+n} \in A_{\alpha} \cap I_{n}$ is a Cohen real over $N_{u}^{\prime}$.

(iii) If $i=0, j=1$ or $i=1, j=0$, then $b_{\omega \delta+n} \in B_{\alpha} \cap I_{n}$ is a Cohen real over $N_{u}^{\prime}$.

The point of using $N_{u}^{\prime}$ rather than $N_{u}$ in (ii) and (iii) is that it will be useful later to have $P(u)$ belonging to the model over which the Cohen reals are chosen.

(6) If $\delta \geq \omega, e_{\delta}$ is a bijective map of $P(\bar{a}\lceil\omega \delta, \bar{b}\lceil\omega \delta)$ onto $\omega \delta$.

(7) For each $\delta^{\prime}$ such that $\delta^{\prime}<\delta, e_{\delta^{\prime}} \subseteq e_{\delta}$.

(8) If $\delta \geq \omega$, the predense subsets of $P(\bar{a} \uparrow \omega \delta, \bar{b} \uparrow \omega \delta)$ which have the form $e_{\delta}^{-1}[S]$ for some $S \subseteq \omega \delta$ such that $S \in \bigcup_{\eta \leq \delta} M_{\eta}$ are predense in $P(\bar{a}\lceil\omega(\delta+1), \bar{b}\lceil\omega(\delta+1))$. 
Remark 5.4. (a) From (1) and (5)((ii)+(iii)), it follows that the sets $\bar{a}^{\delta}$ and $\bar{b}^{\delta}$ are dense in $\mathbb{R}$. From (4)((ii)+(iii)) and (5)((ii)+(iii)), we get $\bar{a}^{\delta} \subseteq A_{\alpha}$ and $\bar{b}^{\delta} \subseteq B_{\alpha}$. From the same clauses together with (5)(i), we get that the enumerations $\left\langle a_{\xi}: \xi<\omega_{1}\right\rangle$ and $\left\langle b_{\xi}: \xi<\omega_{1}\right\rangle$ are one-to-one. (Note that $\alpha(u)=\omega \delta+n$ in (5)(ii) and $\beta(u)=\omega \delta+n$ in (5)(iii).)

(b) From 4(ii) and the fact that $\gamma^{-1}(\alpha, 0)$ is uncountable, it follows that $A_{\alpha} \subseteq$ $\left\{a_{\xi}: \xi<\omega_{1}\right\}$. Similarly, we get $B_{\alpha} \subseteq\left\{b_{\xi}: \xi<\omega_{1}\right\}$.

(c) It follows inductively, using (5)(i) at successor stages, that $\left\{a_{\xi}: \xi<\right.$ $\alpha(u)\} \subseteq N_{u}$ and $\left\{b_{\xi}: \xi<\beta(u)\right\} \subseteq N_{u}$. (At a limit stage $u=(\delta, 0,0)$, we have $\alpha(u)=\beta(u)=\omega \delta$. If $\xi<\omega \delta$, then $\xi=\omega \delta^{\prime}+n^{\prime}$ for some $\delta^{\prime}<\delta$ and $n^{\prime}<\omega$. Then $a_{\xi}$ and $b_{\xi}$ are defined (in an order depending on $\left.\gamma\left(\delta^{\prime}\right)\right)$ at stages $\left(\delta^{\prime}, n^{\prime}, 0\right)$ and $\left(\delta^{\prime}, n^{\prime}, 1\right)$. By the induction hypothesis, $a_{\xi}, b_{\xi} \in N_{\left(\delta^{\prime}, n^{\prime}+1,0\right)} \subseteq N_{u}$.)

Hence, $h_{p} \in N_{u}$ for each $p \in P(u)$.

(d) From (4)(i) and (5)(i), it follows that the sequence $\left\langle N_{u}:(\omega, 0,0) \unlhd u \in Q\right\rangle$ is $\in$-increasing and continuous at limits. This gives in particular that for each limit ordinal $\delta>\omega, P\left(\bar{a}\left\lceil\omega \delta, \bar{b}\lceil\omega \delta)=\bigcup_{\delta^{\prime}<\delta} P\left(\bar{a}\left\lceil\omega \delta^{\prime}, \bar{b}\left\lceil\omega \delta^{\prime}\right)\right.\right.\right.\right.$.

(e) Set $P=P\left(\bar{a} \uparrow \omega_{1}, \bar{b} \uparrow \omega_{1}, \bigcup_{u \in Q} N_{u}\right)$. In the third coordinate we could put the universe (more precisely, $H_{\theta}$ ) since $\bigcup_{u \in Q} N_{u}$ includes all entire functions by (5)(i) and the assumption on the $M_{\delta}$ 's. The conditions (6)(8) ensure that $P$ is $\bar{M}$-cc. To see this, let $e=\bigcup_{\omega \leq \delta<\omega_{1}} e_{\delta}: P \rightarrow \omega_{1}$. For any infinite $\delta<\omega_{1}$ we have $e^{-1}[\omega \delta]=P(\bar{a}\lceil\omega \delta, \bar{b}\lceil\omega \delta)$ and for each $S \subseteq \omega \delta$ belonging to $M_{\delta}$, whenever a set $E$ of the form $e^{-1}[S]=e_{\delta}^{-1}[S]$ is predense in $P\left(\bar{a}\left\lceil\omega \delta, \bar{b}\lceil\omega \delta)\right.\right.$, a simple induction on $\delta^{\prime}$ using (8) shows that if $\delta \leq \delta^{\prime}<\omega_{1}$, then $E$ is predense in $P\left(\bar{a}\left\lceil\omega \delta^{\prime}, \bar{b}\left\lceil\omega \delta^{\prime}\right)\right.\right.$. Thus, $E$ is predense in $P$. For a club of $\delta<\omega_{1}$ we have $\omega \delta=\delta$, so this shows that $P$ satisfies the $\bar{M}$-cc.

We begin by arranging (1)-(7) by induction on $u=(\delta, n, j)$. This is straightforward and we leave most of it to the reader. Notice that because the reals $a_{\xi}$ and $b_{\xi}$ being constructed are not indexed directly by $u$, we need to check that the clauses $(4)((\mathrm{ii})+(\mathrm{iii})),(5)$ and (6) make sense. For 5(ii) for example, it is important that at stage $u, a_{\omega \delta+n}$ has not yet been defined. But at an earlier stage $v=\left(\delta^{\prime}, n^{\prime}, j^{\prime}\right)$, we defined $a_{\omega \delta^{\prime}+n^{\prime}}$ or $b_{\omega \delta^{\prime}+n^{\prime}}$. If $\left(\delta^{\prime}, n^{\prime}\right)$ lexicographically precedes $(\delta, n)$, then $\omega \delta^{\prime}+n^{\prime}<\omega \delta+n$. If $\left(\delta^{\prime}, n^{\prime}\right)=(\delta, n)$, then necessarily $\left(j^{\prime}, j\right)=(0,1)$. Since $j=1$, the assumption of 5 (ii) gives $i=1$. So at stage $v$ we defined $b_{\omega \delta+n}$, not $a_{\omega \delta+n}$. Similarly for 5(iii) and (4)((ii)+(iii)). Similarly, we can check that in (5)(i), $a_{\xi}$ for $\xi<\alpha(u)$ and $b_{\xi}$ for $\xi<\beta(u)$ were defined before stage $u$. For (6), we observe that the partial order $P(\bar{a}\lceil\omega \delta, \bar{b}\lceil\omega \delta)$ is defined because we have reached or passed the stage $(\delta, 0,0)$ and $N_{(\delta, 0,0)}$ has been defined. The function $e_{\delta}$ is chosen at stage $(\delta, 0,0)$. By Remark 5.4(d), the choice of $e_{\delta}$ is dictated by (7) when $\delta>\omega$ is a limit ordinal. The function $e_{\delta+1}$ can be taken to be an arbitrary extension of $e_{\delta}$ satisfying (6).

We must check that the construction gives (8). Let $E$ be a predense subset of $P\left(\bar{a}\left\lceil\omega \delta, \bar{b}\lceil\omega \delta)\right.\right.$ of the appropriate form, i.e., $E=e_{\delta}^{-1}[S]$ for some $S \subseteq \omega \delta$ such that $S \in \bigcup_{\eta \leq \delta} M_{\eta}$. We will show by induction on $u=(\delta, n, j) \in Q$ such that $(\delta, 0,0) \unlhd u \triangleleft(\delta+1,0,0)$ that $E$ remains predense in $P(u+1)$, where $u+1$ denotes the successor of $u$ in $Q$, i.e., $(\delta, n, 1)$ if $j=0$ and $(\delta, n+1,0)$ if $j=1$. 
(This establishes (8) since each member of $P(\bar{a}\lceil\omega(\delta+1), \bar{b}\lceil\omega(\delta+1))$ belongs to $P(\bar{a}\lceil\omega \delta+n, \bar{b}\lceil\omega \delta+n)$ for some $n<\omega)$.

Remark 5.5. At the stage where $n=0$ and $j=0$, we consider the passage from $P(\bar{a}\lceil\omega \delta, \bar{b}\lceil\omega \delta)$ to either $P(\bar{a}\lceil\omega \delta+1, \bar{b}\lceil\omega \delta)$ or $P(\bar{a}\lceil\omega \delta, \bar{b}\lceil\omega \delta+1)$ (depending on whether $i=0$ or $i=1)$. These two partial orders have the same allowable finite parts $h_{p}$ for their conditions, because, by Definition 5.2 (ii), there is no legal value for either of $a_{\omega \delta}$ or $b_{\omega \delta}$ to correspond to until the other is chosen.

Let $p \in P(u+1) \backslash P(u)$. We must show that $p$ is compatible with some member of $E$.

Case 1. $h_{p} \in N_{u}$.

Proposition 4.5 gives a function $f^{\prime} \in\left(f_{0}+H \operatorname{span} \mathcal{G}_{0}\right) \cap N_{u}$ such that

1(a) $h_{p} \subseteq f^{\prime}$;

1 (d) for all $i<\omega$ and all $x \in \mathbb{R}$ such that $|x| \geq c_{i},\left|D^{i} f_{p}(x)-D^{i} f^{\prime}(x)\right|<\frac{1}{2} \varepsilon_{p} \eta(x)$ (and hence, using Definition 5.2(v), $\left.\left|D^{i}\left(f^{\prime}-f_{0}\right)(x)\right|<\left(1-\frac{1}{2} \varepsilon_{p}\right) \eta(x)\right)$;

1 (f) for all $z \in \mathbb{C}$ such that $|z| \leq n_{p},\left|f_{p}(z)-f^{\prime}(z)\right|<\varepsilon_{p} / 2$.

The letters in the labels here correspond to those in the statement of Proposition 4.5. The number 1 is a reference to Case 1 . We will use similar notation in the rest of the proof when applying this proposition. (To get $f^{\prime}$, in Proposition 4.5. take $\varepsilon(x)=\eta(x)$, but get the conclusion for $\frac{1}{2} \varepsilon_{p} \eta$ instead of $\eta$. See Remark 4.6, Note that $\frac{1}{2} \varepsilon_{p} \eta \leq \frac{1}{2} \varepsilon_{p}$ because $\eta \leq 1$.) Then

$$
q=\left(h_{p}, f^{\prime}, \varepsilon_{p} / 2, n_{p}\right)
$$

belongs to $P(u)$. Also, $q$ and some $r \in E$ have a common extension $q^{\prime} \in P(u)$. Then $q^{\prime} \leq p$ since for each $z \in \mathbb{C}$ such that $|z| \leq n_{p}$, we have also $|z| \leq n_{q}$ (since $\left.n_{q}=n_{p}\right)$ and hence

$$
\begin{aligned}
\left|f_{q^{\prime}}(z)-f_{p}(z)\right|+\varepsilon_{q^{\prime}} & \leq\left|f_{q}(z)-f_{p}(z)\right|+\left|f_{q^{\prime}}(z)-f_{q}(z)\right|+\varepsilon_{q^{\prime}} \\
& =\left|f^{\prime}(z)-f_{p}(z)\right|+\left|f_{q^{\prime}}(z)-f_{q}(z)\right|+\varepsilon_{q^{\prime}} \\
& \leq \varepsilon_{p} / 2+\left|f_{q^{\prime}}(z)-f_{q}(z)\right|+\varepsilon_{q^{\prime}} \\
& \leq \varepsilon_{p} / 2+\varepsilon_{q}=\varepsilon_{p} .
\end{aligned}
$$

Case 2. $h_{p} \notin N_{u}$.

By Remark 5.5, we have $(n, j) \neq(0,0)$. Hence (by (5)(i)), $e_{\delta},\left\langle M_{\delta^{\prime}}: \delta^{\prime} \leq \delta\right\rangle \in N_{u}$ which gives $S \in N_{u}$ and hence $E=e_{\delta}^{-1}[S] \in N_{u}$.

Subcase 2a. $i=0, j=1$ or $i=1, j=0$.

In this subcase, $h_{p}$ has the form $h \cup\left\{\left(a, b_{\omega \delta+n}\right)\right\}$ for some $h \subseteq N_{u}$ and $a \in$ $\left\{a_{\omega \delta+m}: m<n+1-i\right\}$.

Proposition 4.5 gives $n_{1} \in \mathbb{N}$, a function $f^{\prime} \in\left(f_{0}+H \operatorname{span} \mathcal{G}_{0}\right) \cap N_{u}$ and a rational number $\lambda_{0}>0$ such that

2a(a) $h \subseteq f^{\prime}$;

$2 \mathrm{a}(\mathrm{d}) n_{1} \geq 4|\operatorname{dom} h|$ and for all $i<\omega$, for all $x \in \mathbb{R}$ such that $|x| \geq c_{i}$ and for all $\lambda \in \mathbb{R}$ such that $|\lambda| \leq \lambda_{0}$

$$
\left|D^{i} f_{p}(x)-D^{i}\left(f^{\prime}+\lambda H g\left(n_{1}, \operatorname{dom} h\right)\right)(x)\right|<\frac{1}{2} \varepsilon_{p} \eta(x)
$$

(and hence in particular $\left.\left|D^{i}\left(f^{\prime}+\lambda H g\left(n_{1}, \operatorname{dom} h\right)-f_{0}\right)(x)\right|<\left(1-\frac{1}{2} \varepsilon_{p}\right) \eta(x)\right)$;

$2 \mathrm{a}$ (e) $g\left(n_{1}, \operatorname{dom} h\right)(a) \neq 0$ and for some number $\lambda$ such that $|\lambda|<\lambda_{0}$, we have $h_{p} \subseteq f^{\prime}+\lambda H g\left(n_{1}, \operatorname{dom} h\right)$ 
$2 \mathrm{a}(\mathrm{f})$ for all $z \in \mathbb{C}$ such that $|z| \leq n_{p}$ we have $\lambda_{0}\left|H(z) g\left(n_{1}, \operatorname{dom} h\right)(z)\right|<\varepsilon_{p} / 4$ and for all $\lambda$ such that $|\lambda|<\lambda_{0},\left|f_{p}(z)-\left(f^{\prime}+\lambda H g\left(n_{1}, \operatorname{dom} h\right)\right)(z)\right|<\varepsilon_{p} / 4$.

For functions $g_{0}: \mathbb{C} \rightarrow \mathbb{C}$ such that $g_{0}[\mathbb{R}] \subseteq \mathbb{R}$, numbers $\mu>0, m \in \mathbb{N}$ and finite sets $A \subseteq \mathbb{R}$, define

$$
V\left(g_{0}, \mu, m, A\right)=\left\{\left(g_{0}+\lambda H g(m, A)\right)(a):|\lambda|<\mu\right\} .
$$

As long as $g(m, A)(a) \neq 0, V\left(g_{0}, \mu, m, A\right)$ is a nonempty open interval in $\mathbb{R}$. Consider the open interval $U=V\left(f^{\prime}, \lambda_{0}, n_{1}, \operatorname{dom} h\right)$. By $2 \mathrm{a}(\mathrm{e}), f_{p}(a)=b_{\omega \delta+n} \in U$. Define

$$
q_{0}=\left(h, f^{\prime}, \varepsilon_{p} / 2, n_{p}\right)
$$

and notice that $q_{0} \in P(u)$.

Claim 5.6. The union of the open sets $V\left(f_{q}, \mu, m, \operatorname{dom} h_{q}\right)$ such that

(1) $q \in P(u)$ is a common extension of $q_{0}$ and an element of $E$,

(2) $g\left(m, \operatorname{dom} h_{q}\right)(a) \neq 0$,

(3) $\mu>0$ is rational, $m \in \mathbb{N}, m \geq 4\left|\operatorname{dom} h_{q}\right|$,

(4) $V\left(f_{q}, \mu, m, \operatorname{dom} h_{q}\right) \subseteq U$,

(5) for all $z \in \mathbb{C}$ such that $|z| \leq n_{q}, \mu \mid H(z) g\left(m\right.$, dom $\left.h_{q}\right)(z) \mid \leq \varepsilon_{q} / 2$ and for all $i<\omega$, for all $x \in \mathbb{R}$ such that $|x| \geq c_{i}, \mu\left|D^{i}\left(H g\left(m, \operatorname{dom} h_{q}\right)\right)(x)\right|<\frac{1}{2} \varepsilon_{q} \eta(x)$ (and hence for all $\lambda$ such that $|\lambda| \leq \mu$ we have

$$
\left.\left|D^{i}\left(f_{q}+\lambda H g\left(m, \operatorname{dom} h_{q}\right)-f_{0}\right)(x)\right|<\left(1-\frac{1}{2} \varepsilon_{q}\right) \eta(x)\right)
$$

is dense in $U$.

Proof of Claim 5.6. Fix $\lambda_{1} \in \mathbb{Q}$ such that $\left|\lambda_{1}\right|<\lambda_{0}$. Define

$$
w=\bar{f}^{\prime}(a) \in U, \text { where } \bar{f}^{\prime}=f^{\prime}+\lambda_{1} H g\left(n_{1}, \operatorname{dom} h\right) .
$$

Note that the numbers $w$ of this form, as $\lambda_{1}$ runs over all rational numbers such that $\left|\lambda_{1}\right|<\lambda_{0}$, are dense in $U$. Fix $\delta>0$ such that $(w-\delta, w+\delta) \subseteq U$. Let $x_{1}, x_{2} \in A_{0} \backslash$ dom $h$ satisfy $x_{1}<a<x_{2}$ and $\bar{f}^{\prime}\left(x_{1}\right), \bar{f}^{\prime}\left(x_{2}\right) \in(w-\delta, w+\delta)$. Apply Proposition 4.5 to get a function $f^{\prime \prime} \in\left(f_{0}+H \operatorname{span} \mathcal{G}_{0}\right) \cap N_{u}$ and a rational number $\lambda_{2}>0$ such that

5.6.(a) $h \subseteq f^{\prime \prime}$,

[5.6(b) $y_{1}=f^{\prime \prime}\left(x_{1}\right)$ and $y_{2}=f^{\prime \prime}\left(x_{2}\right)$ are both members of $B_{0}$,

5.6(d) $)_{1} y_{1}, y_{2} \in(w-\delta, w+\delta)$.

(This holds as long as the quantities $\left|\left(\bar{f}^{\prime}-f^{\prime \prime}\right)\left(x_{j}\right)\right|, j=1,2$, are small enough.)

5.6(d) $)_{2}$ For all $i<\omega$ and for all $x \in \mathbb{R}$ such that $|x| \geq c_{i}$,

$$
\left|D^{i} \bar{f}^{\prime}(x)-D^{i} f^{\prime \prime}(x)\right|<\frac{1}{4} \varepsilon_{p} \eta(x)
$$

(and hence in particular $\left.\left|D^{i}\left(f^{\prime \prime}-f_{0}\right)(x)\right|<\left(1-\frac{1}{4} \varepsilon_{p}\right) \eta(x)\right)$.

5.6(f) For all $z \in \mathbb{C}$ such that $|z| \leq n_{p},\left|\bar{f}^{\prime}(z)-f^{\prime \prime}(z)\right|<\varepsilon_{p} / 8$.

Note that $f^{\prime \prime}\lceil\mathbb{R} \text { is increasing (by [5.6(d) })_{2}$, for all $x \in \mathbb{R}\left|D f^{\prime \prime}(x)-D f_{0}(x)\right|<\eta(x) \leq$ $D f_{0}(x)$ and hence $\left.D f^{\prime \prime}(x)>0\right)$ and hence we have $y_{1}<y_{2}$. Then $q_{1} \in P(u)$, where

$$
q_{1}=\left(h \cup\left\{\left(x_{1}, y_{1}\right),\left(x_{2}, y_{2}\right)\right\}, f^{\prime \prime}, \varepsilon_{p} / 8, n_{p}\right) .
$$


Also, $q_{1}$ extends $q_{0}$ because if $|z| \leq n_{p}$, then

$$
\begin{aligned}
\left|f_{q_{1}}(z)-f_{q_{0}}(z)\right|+\varepsilon_{q_{1}} & =\left|f^{\prime \prime}(z)-f^{\prime}(z)\right|+\varepsilon_{p} / 8 \\
& \leq\left|f^{\prime \prime}(z)-\bar{f}^{\prime}(z)\right|+\left|\bar{f}^{\prime}(z)-f^{\prime}(z)\right|+\varepsilon_{p} / 8 \\
& \leq\left|\bar{f}^{\prime}(z)-f^{\prime}(z)\right|+\varepsilon_{p} / 4 \leq \varepsilon_{p} / 2=\varepsilon_{q_{0}} .
\end{aligned}
$$

By the induction hypothesis, there is a common extension $q \in P(u)$ of $q_{1}$ and some $r \in E$. Since $a$ does not belong to the domains of $h_{q_{1}}$ or $h_{r}(r \in E \subseteq P(\delta, 0,0)$ and hence $\left.\operatorname{dom} h_{r} \subseteq\left\{a_{\xi}: \xi<\omega \delta\right\}\right)$, we may discard it from the domain of $h_{q}$ if necessary to get $a \notin \operatorname{dom} h_{q}$. Then choose $m \geq 4\left|\operatorname{dom} h_{q}\right|$ large enough so that part 2 of the claim holds. For $\mu>0$ small enough we have that part 5 of the claim holds.

[For the first assertion of part 5 this is clear. For the second, proceed as follows. By Proposition 4.4(c), whenever $|x| \geq c_{i}$ we have $\left|D^{i}\left(H g\left(m, \operatorname{dom} h_{q}\right)\right)(x)\right| \leq$ $(3 / 4)^{i} \zeta(x) \eta(x)$. For all large enough $i$, say $i>i_{0}$, we have $(3 / 4)^{i} \leq \frac{1}{2} \varepsilon_{q}$ and hence, for $|x| \geq c_{i},\left|D^{i}\left(H g\left(m, \operatorname{dom} h_{q}\right)\right)(x)\right| \leq(3 / 4)^{i} \zeta(x) \eta(x) \leq \frac{1}{2} \varepsilon_{q} \eta(x)$. Moreover, for all $x$ outside some compact interval $L$ we have $\zeta(x) \leq \frac{1}{2} \varepsilon_{q}$ and hence, for $|x| \geq c_{i}$, $\left|D^{i}\left(H g\left(m, \operatorname{dom} h_{q}\right)\right)(x)\right| \leq(3 / 4)^{i} \zeta(x) \eta(x) \leq \frac{1}{2} \varepsilon_{q} \eta(x)$. For $\mu$ small enough we will also have $\mu\left|D^{i}\left(H g\left(m, \operatorname{dom} h_{q}\right)\right)(x)\right|<\frac{1}{2} \varepsilon_{q} \eta(x)$ for each $i \leq i_{0}$ and each $x \in L$, giving part 5 of the claim.]

Then the functions in the definition of $V\left(f_{q}, \mu, m, \operatorname{dom} h_{q}\right)$ are increasing on $\mathbb{R}$. Since $\left\{\left(x_{1}, y_{1}\right),\left(x_{2}, y_{2}\right)\right\} \subseteq h_{q}$, we have $V\left(f_{q}, \mu, m, \operatorname{dom} h_{q}\right) \subseteq\left(y_{1}, y_{2}\right) \subseteq(w-\delta, w+$ $\delta) \subseteq U$ and, in particular, part 4 of the claim holds.

This proves Claim 5.6 .

The dense open subset of $U$ given by Claim 5.6 is coded in $N_{u}^{\prime}$. (As noted earlier, $P(u) \in N_{u}^{\prime}$ when $u$ is not a limit stage.) By (5)(iii), there are $q, \mu, m$ satisfying Claim [5.6(1-5) for which $b_{\omega \delta+n} \in V\left(f_{q}, \mu, m\right.$, dom $\left.h_{q}\right)$. Choosing $\lambda$ with $|\lambda|<\mu$ so that

$$
\left(f_{q}+\lambda H g\left(m, \operatorname{dom} h_{q}\right)\right)(a)=b_{\omega \delta+n}
$$

(note that this equation uniquely determines $\lambda$ and hence $\lambda \in N_{u+1}$ ), we get that

$$
q^{\prime}=\left(h_{q} \cup\left\{\left(a, b_{\omega \delta+n}\right)\right\}, f_{q}+\lambda H g\left(m, \operatorname{dom} h_{q}\right), \varepsilon_{q} / 2, n_{q}\right)
$$

belongs to $P(u+1)$ (using the second part of clause 5 of Claim [5.6) and extends both $q$ and $p$. It extends $q$ by the first part of clause 5 of Claim 5.6. To see that $q^{\prime} \leq p$, note that for each $z \in \mathbb{C}$ such that $|z| \leq n_{p}$,

$$
\begin{aligned}
\left|f_{q^{\prime}}(z)-f_{p}(z)\right|+\varepsilon_{q^{\prime}} & \leq\left|f_{q}(z)-f_{p}(z)\right|+\left|f_{q^{\prime}}(z)-f_{q}(z)\right|+\varepsilon_{q^{\prime}} \\
& \leq\left|f_{q}(z)-f_{p}(z)\right|+\varepsilon_{q} \\
& \leq\left|f_{q_{0}}(z)-f_{p}(z)\right|+\left|f_{q}(z)-f_{q_{0}}(z)\right|+\varepsilon_{q} \\
& \leq\left|f_{q_{0}}(z)-f_{p}(z)\right|+\varepsilon_{q_{0}} \\
& \leq \varepsilon_{p} / 4+\varepsilon_{p} / 2<\varepsilon_{p} .
\end{aligned}
$$

Thus, $p$ is compatible with $q$ and hence with some element of $E$.

Subcase $2 \mathrm{~b}$. $i=0, j=0$ or $i=1, j=1$. This subcase is similar to the previous one. In the setting of [Sh1980], the corresponding two subcases are symmetric. In our context they are not, however, because the entire functions $f_{p}$ are not invertible.

In this subcase, $h_{p}$ has the form $h \cup\left\{\left(a_{\omega \delta+n}, b\right)\right\}$ for some $h \subseteq N_{u}$ and $b \in$ $\left\{b_{\omega \delta+m}: m<n+i\right\}$. 
Proposition 4.5 gives $n_{1} \in \mathbb{N}$, a function $f^{\prime} \in\left(f_{0}+H\right.$ span $\left.\mathcal{G}_{0}\right) \cap N_{u}$ and a rational number $\lambda_{0}>0$ such that

$2 \mathrm{~b}$ (a) $h \subseteq f^{\prime}$;

$2 \mathrm{~b}(\mathrm{~d}) n_{1} \geq 4|\operatorname{dom} h|$ and for all $i<\omega$, for all $x \in \mathbb{R}$ such that $|x| \geq c_{i}$ and for all $\lambda \in \mathbb{R}$ such that $|\lambda| \leq \lambda_{0}$

$$
\left|D^{i} f_{p}(x)-D^{i}\left(f^{\prime}+\lambda H g\left(n_{1}, \operatorname{dom} h\right)\right)(x)\right|<\frac{1}{2} \varepsilon_{p} \eta(x)
$$

(and hence in particular $\left.\left|D^{i}\left(f^{\prime}+\lambda H g\left(n_{1}, \operatorname{dom} h\right)-f_{0}\right)(x)\right|<\left(1-\frac{1}{2} \varepsilon_{p}\right) \eta(x)\right)$;

$2 \mathrm{~b}(\mathrm{e})$ for some number $\lambda$ such that $|\lambda|<\lambda_{0}$, we have $h_{p} \subseteq f^{\prime}+\lambda H g\left(n_{1}, \operatorname{dom} h\right)$;

$2 \mathrm{~b}(\mathrm{f})$ for all $z \in \mathbb{C}$ such that $|z| \leq n_{p}$ we have $\lambda_{0}\left|H(z) g\left(n_{1}, \operatorname{dom} h\right)(z)\right|<\varepsilon_{p} / 4$ and for all $\lambda$ such that $|\lambda|<\lambda_{0},\left|f_{p}(z)-\left(f^{\prime}+\lambda H g\left(n_{1}, \operatorname{dom} h\right)\right)(z)\right|<\varepsilon_{p} / 4$.

(This list agrees with the one for Subcase 2a except for (e).)

Remark 5.7. Note that $2 \mathrm{~b}(\mathrm{~d})$ ensures that for $|\lambda| \leq \lambda_{0}$, the restriction to $\mathbb{R}$ of $f^{\prime}+\lambda H g\left(n_{1}, \operatorname{dom} h\right)$ is an order-isomorphism. Indeed, as in the argument after 5.6(f) above, $f^{\prime}+\lambda H g\left(n_{1}, \operatorname{dom} h\right)$ is increasing. The fact that for $|x| \geq c_{0}$ we have $\left|\left(f^{\prime}+\lambda H g\left(n_{1}, \operatorname{dom} h\right)\right)(x)-f_{0}(x)\right|<\eta(x) \leq 1$ ensures that $f^{\prime}+\lambda H g\left(n_{1}, \operatorname{dom} h\right)$ is onto.

For functions $g_{0}: \mathbb{C} \rightarrow \mathbb{C}$ such that $g_{0}[\mathbb{R}] \subseteq \mathbb{R}$, numbers $\mu>0, m \in \mathbb{N}$ and finite sets $A \subseteq \mathbb{R}$, define

$$
W\left(g_{0}, \mu, m, A\right)=\left\{\left(g_{0}+\lambda H g(m, A)\right)^{-1}(b):|\lambda|<\mu\right\} .
$$

The definition makes sense if the restriction to $\mathbb{R}$ of $g_{0}+\lambda H g(m, A)$ is an orderisomorphism whenever $|\lambda|<\mu$. (In the definition, $\left(g_{0}+\lambda H g(m, A)\right)^{-1}$ means the inverse of this restriction.) By Proposition 4.1, $W\left(g_{0}, \mu, m, A\right)$ is an open interval in $\mathbb{R}$ as long as there is no $a \in \mathbb{R}$ such that $g_{0}(a)=b$ and $g(m, A)(a)=0$. When $g_{0} \uparrow \mathbb{R}$ is invertible, then only the value $a=g_{0}^{-1}(b)$ is relevant. Consider the open interval $U=W\left(f^{\prime}, \lambda_{0}, n_{1}\right.$, dom $\left.h\right)$. By $(\mathrm{e}), a_{\omega \delta+n}=f_{p}^{-1}(b) \in U$. Define

$$
q_{0}=\left(h, f^{\prime}, \varepsilon_{p} / 2, n_{p}\right)
$$

and notice that $q_{0} \in P(u)$.

Claim 5.8. The union of the open sets $W\left(f_{q}, \mu, m, \operatorname{dom} h_{q}\right)$ such that

(1) $q \in P(u)$ is a common extension of $q_{0}$ and an element of $E$;

(2) $g\left(m, \operatorname{dom} h_{q}\right)(a) \neq 0$ where $a=f_{q}^{-1}(b)$;

(3) $\mu>0$ is rational, $m \in \mathbb{N}, m \geq 4\left|\operatorname{dom} h_{q}\right|$;

(4) $W\left(f_{q}, \mu, m, \operatorname{dom} h_{q}\right) \subseteq U$;

(5) for all $z \in \mathbb{C}$ such that $|z| \leq n_{q}, \mu\left|H(z) g\left(m, \operatorname{dom} h_{q}\right)(z)\right| \leq \varepsilon_{q} / 2$ and for all $i<\omega$, for all $x \in \mathbb{R}$ such that $|x| \geq c_{i}, \mu\left|D^{i}\left(H g\left(m, \operatorname{dom} h_{q}\right)\right)(x)\right|<\frac{1}{2} \varepsilon_{q} \eta(x)$ (and hence for all $\lambda$ such that $|\lambda| \leq \mu$,

$$
\left.\left|D^{i}\left(f_{q}+\lambda H g\left(m, \operatorname{dom} h_{q}\right)-f_{0}\right)(x)\right|<\left(1-\frac{1}{2} \varepsilon_{q}\right) \eta(x)\right)
$$

is dense in $U$.

Proof of Claim 5.8. Fix $\lambda_{1} \in \mathbb{Q}$ such that $\left|\lambda_{1}\right|<\lambda_{0}$. Define

$$
w=\left(\bar{f}^{\prime}\right)^{-1}(b) \in U, \text { where } \bar{f}^{\prime}=f^{\prime}+\lambda_{1} H g\left(n_{1}, \operatorname{dom} h\right) .
$$

By Remark [5.7] $\bar{f}^{\prime}$ is an order-isomorphism. Note that $w \notin \operatorname{dom} h$ since $b \notin$ range $h$ and $h \subseteq \bar{f}^{\prime}$. Note that the numbers $w$ of the given form, as $\lambda_{1}$ runs over all rational numbers such that $\left|\lambda_{1}\right|<\lambda_{0}$, are dense in $U$. Fix $\delta>0$ such that $(w-\delta, w+\delta) \subseteq$ 
$U$ and $(w-\delta, w+\delta) \cap \operatorname{dom} h=\emptyset$. Let $y_{1}, y_{2} \in B_{0}$ satisfy $y_{1}<b<y_{2}$ and $\left(\bar{f}^{\prime}\right)^{-1}\left(y_{1}\right),\left(\bar{f}^{\prime}\right)^{-1}\left(y_{2}\right) \in(w-\delta, w+\delta)$. Apply Proposition 4.5 to get $n_{2} \in \mathbb{N}$, a function $f^{\prime \prime} \in\left(f_{0}+H \operatorname{span} \mathcal{G}_{0}\right) \cap N_{u}$ and a rational number $\lambda_{2}>0$ such that

5.8(a) $h \subseteq f^{\prime \prime}$;

[5.8(c) $y_{1}=f^{\prime \prime}\left(x_{1}\right)$ and $y_{2}=f^{\prime \prime}\left(x_{2}\right)$, where $x_{1}, x_{2}$ are both members of $A_{0}$ and $x_{1}, x_{2} \in(w-\delta, w+\delta)$

5.8(d) for all $i<\omega$ and for all $x \in \mathbb{R}$ such that $|x| \geq c_{i}$,

$$
\left|D^{i} \bar{f}^{\prime}(x)-D^{i} f^{\prime \prime}(x)\right|<\frac{1}{4} \varepsilon_{p} \eta(x)
$$

(and hence in particular $\left.\left|D^{i}\left(f^{\prime \prime}-f_{0}\right)(x)\right|<\left(1-\frac{1}{4} \varepsilon_{p}\right) \eta(x)\right)$;

[5.8(f) for all $z \in \mathbb{C}$ such that $|z| \leq n_{p},\left|\bar{f}^{\prime}(z)-f^{\prime \prime}(z)\right|<\varepsilon_{p} / 8$.

By 5.8(d), $f^{\prime \prime} \uparrow \mathbb{R}$ is increasing and hence $x_{1}<x_{2}$. Then $q_{1} \in P(u)$, where

$$
q_{1}=\left(h \cup\left\{\left(x_{1}, y_{1}\right),\left(x_{2}, y_{2}\right)\right\}, f^{\prime \prime}, \varepsilon_{p} / 8, n_{p}\right) .
$$

Exactly as in the proof of Claim [5.6, $q_{1}$ extends $q_{0}$. By the induction hypothesis, there is a common extension $q \in P(u)$ of $q_{1}$ and some $r \in E$. The number $a=$ $f_{q}^{-1}(b)$ does not belong to the domains of $h_{q_{1}}$ or $h_{r}$.

[From the fact that $f_{q} \uparrow \mathbb{R}$ is increasing and hence injective, we see that because $f_{q}\left(x_{i}\right)=y_{i}, i=1,2$, and $f_{q}(a)=b$, we have $a \neq x_{i}, i=1,2$. Because $h \subseteq f_{q}$ and $b \notin$ range $h$, we have $a \notin \operatorname{dom} h$. Thus, $a \notin \operatorname{dom} h_{q_{1}}$. Since $h_{r} \subseteq h_{q} \subseteq f_{q}$, if $a$ were in dom $h_{r}$, then $h_{r}(a)=b$, which is not possible because $r \in E \subseteq P(\delta, 0,0)$ and hence range $h_{r} \subseteq\left\{b_{\xi}: \xi<\omega \delta\right\}$.

We may thus discard it from the domain of $h_{q}$ if necessary to get $a \notin \operatorname{dom} h_{q}$. Choose $m \geq 4\left|\operatorname{dom} h_{q}\right|$ large enough so that part 2 of the claim holds. As in the proof of Claim 5.6. for $\mu>0$ small enough we have that part 5 of the claim holds. Then the functions in the definition of $W\left(f_{q}, \mu, m, \operatorname{dom} h_{q}\right)$ are order-isomorphisms. Since $\left\{\left(x_{1}, y_{1}\right),\left(x_{2}, y_{2}\right)\right\} \subseteq h_{q}$, we have $W\left(f_{q}, \mu, m, \operatorname{dom} h_{q}\right) \subseteq\left(x_{1}, x_{2}\right) \subseteq$ $(w-\delta, w+\delta) \subseteq U$ and in particular part 4 of the claim holds.

This proves Claim 5.8

The dense open subset of $U$ given by Claim 5.8 is coded in $N_{u}^{\prime}$. By (5)(ii), there are $q, \mu, m$ satisfying Claim $5.8(1-5)$ for which $a_{\omega \delta+n} \in W\left(f_{q}, \mu, m, \operatorname{dom} h_{q}\right)$. Choosing $\lambda$ with $|\lambda|<\mu$ so that $\left(f_{q}+\lambda H g\left(m, \operatorname{dom} h_{q}\right)\right)\left(a_{\omega \delta+n}\right)=b$, we get, as in Subcase $2 \mathrm{a}$, that

$$
q^{\prime}=\left(h_{q} \cup\left\{\left(a_{\omega \delta+n}, b\right)\right\}, f_{q}+\lambda H g\left(m, \operatorname{dom} h_{q}\right), \varepsilon_{q} / 2, n_{q}\right)
$$

belongs to $P(u+1)$ and extends both $q$ and $p$. Thus, $p$ is compatible with $q$ and hence with some element of $E$. This completes the proof of (8).

We now have an $\bar{M}$-cc partial order $P=P\left(\bar{a}\left\lceil\omega_{1}, \bar{b}\left\lceil\omega_{1}\right)\right.\right.$ as in Remark 5.4(e). It remains to check that forcing with $P$ adds the desired entire function $f$. Let $h=\bigcup\left\{h_{p}: p \in G\right\}$. For each $\alpha<\omega_{1}$ and each $x \in A_{\alpha}$, it follows using Proposition 4.5(b) that conditions with $f_{p}(x) \in B_{\alpha}$ are dense and then (by extending such conditions further) so are conditions with $x \in \operatorname{dom} h_{p}$. Similarly, for each $\alpha<\omega_{1}$ and each $y \in B_{\alpha}$, the conditions with $y \in$ range $h_{p}$ are dense (using Proposition 4.5(c) this time). Hence $\operatorname{dom} h=\bigcup_{\alpha<\omega_{1}} A_{\alpha}$, range $h=\bigcup_{\alpha<\omega_{1}} B_{\alpha}$ and $h$ is clearly an order-isomorphism. For $k \in \mathbb{N}$, choose $p_{k} \in G$ such that $p_{k+1} \leq p_{k}$, $n_{p_{k}} \geq k$ and $\varepsilon_{p_{k}}<1 / k$. The sequence $\left\{f_{p_{k}}\right\}$ is uniformly Cauchy on compact sets because for $\ell>k$ and $|z| \leq k\left(\leq n_{p_{k}}\right),\left|f_{p_{\ell}}(z)-f_{p_{k}}(z)\right| \leq \varepsilon_{p_{k}}<1 / k$. Define $f: \mathbb{C} \rightarrow$ $\mathbb{C}$ by $f(z)=\lim _{k \rightarrow \infty} f_{p_{k}}(z)$. For all $i<\omega$, we have $D^{i} f(z)=\lim _{k \rightarrow \infty} D^{i} f_{p_{k}}(z)$ 
uniformly on compact sets [Ru, Theorem 10.28]. Also, for each $a \in \bigcup_{\alpha<\omega_{1}} A_{\alpha}$, we can choose $k$ such that $|a| \leq n_{p_{k}}$, and $p \in G$ such that $p \leq p_{k}$ and $a \in \operatorname{dom} h_{p}$. Then

$$
\left|f_{p_{k}}(a)-h(a)\right|=\left|f_{p_{k}}(a)-h_{p}(a)\right|=\left|f_{p_{k}}(a)-f_{p}(a)\right| \leq \varepsilon_{p_{k}}<1 / k,
$$

and hence $f(a)=\lim _{k \rightarrow \infty} f_{p_{k}}(a)=h(a)$. Also, for each $i<\omega$ and each $x \in \mathbb{R}$ such that $|x| \geq c_{i}$,

$$
\left|D^{i} f(x)-D^{i} f_{0}(x)\right|=\lim _{k \rightarrow \infty}\left|D^{i} f_{p_{k}}(x)-D^{i} f_{0}(x)\right| \leq \eta(x) .
$$

This completes the proof of the lemma.

\section{Proof of Theorem 1.7}

By Proposition 3.6, it suffices to prove the theorem in the case where the function $g$ in part (c) is the restriction to $\mathbb{R}$ of an entire function and has a strictly positive derivative. Note that in this case, (c)(i) can be omitted as it is covered by (c)(ii). The rest of the proof is standard oracle-cc technique. We sketch the argument. Our sketch is an adaptation to the present context of the corresponding argument in [BM]. Start with a ground model of $V=L$. Fix a diamond sequence

$$
\left\langle\left(x_{\alpha}, a_{\alpha}, b_{\alpha}, f_{\alpha}, c_{\alpha}, e_{\alpha}\right): \alpha<\omega_{2}, \operatorname{cof}(\alpha)=\omega_{1}\right\rangle
$$

for trapping sextuples $(x, a, b, f, c, e)$ consisting of:

(1) A function $x: \omega_{2} \rightarrow\left(\left[\omega_{2}\right] \leq \omega\right) \omega$. The idea of $x$ is that, with $\omega_{2}$ identified with the ccc partial order we are about to build, $\left[\omega_{2}\right] \leq \omega$ contains the antichains. Thus, $\left(\left[\omega_{2}\right] \leq \omega\right) \omega$ contains a name for each real number (construed as a subset of $\omega)$. Then for any second category set $X$ in the extension, we can find a ground model function $x: \omega_{2} \rightarrow\left(\left[\omega_{2}\right] \leq \omega\right)^{\omega}$ enumerating the names of the elements of $X$.

(2) Functions $a, b: \omega_{1} \times \omega_{1} \rightarrow\left(\left[\omega_{2}\right]^{\leq \omega}\right)^{\omega}$ representing (enumerations of the names for the elements of) an $\omega_{1}$-sequence of sets in which the first $\omega$, represented by $\{a(i, n): n<\omega\}$ and $\{b(i, n): n<\omega\}, i<\omega$, are countable dense sets and the remainder, represented by $\left\{a(\alpha, \xi): \xi<\omega_{1}\right\}$ and $\left\{b(\alpha, \xi): \xi<\omega_{1}\right\}, \omega \leq \alpha<\omega_{1}$, are everywhere second category sets of cardinality $\omega_{1}$.

(3) A function $f \in\left(\left[\omega_{2}\right]^{\leq \omega}\right)^{\omega}$ representing a name for the Borel code of an entire function restricting to an order-isomorphism of $\mathbb{R}$ having a strictly positive derivative.

(4) A function $c: \omega \rightarrow\left(\left[\omega_{2}\right]^{\leq \omega}\right)^{\omega}$ so that $\langle c(i): i<\omega\rangle$ represents a sequence of names for the terms of a nondecreasing sequence of nonnegative real numbers converging to $\infty$.

(5) A function $e \in\left(\left[\omega_{2}\right] \leq \omega\right)^{\omega}$ intended to represent a name for the Borel code of a positive continuous function.

So for each $\alpha<\omega_{2}$ of cofinality $\omega_{1}$, we have $x_{\alpha}: \alpha \rightarrow\left([\alpha]^{\leq \omega}\right)^{\omega}, a_{\alpha}, b_{\alpha}: \omega_{1} \times \omega_{1} \rightarrow$ $\left([\alpha]^{\leq \omega}\right)^{\omega}, f_{\alpha}, e_{\alpha} \in\left([\alpha]^{\leq \omega}\right)^{\omega}$ and $c_{\alpha}: \omega \rightarrow\left([\alpha]^{\leq \omega}\right)^{\omega}$. Also, for each $(x, a, b, f, c, e)$ as in (1)-(5), $\left\{\alpha<\omega_{2}: \operatorname{cof}(\alpha)=\omega_{1}, x\left\lceil\alpha=x_{\alpha}, a=a_{\alpha}, b=b_{\alpha}, f=f_{\alpha}, c=c_{\alpha}\right.\right.$ and $\left.e=e_{\alpha}\right\}$ is stationary in $\omega_{2}$.

We will inductively define an $\omega_{2}$-stage finite support iteration

$$
\left\langle\left\langle P_{\alpha}\right\rangle_{\alpha \leq \omega_{2}},\left\langle\dot{Q}_{\alpha}\right\rangle_{\alpha<\omega_{1}}\right\rangle
$$


as well as $P_{\alpha}$-names $\bar{M}_{\alpha}$ for oracles and one-to-one functions $F_{\alpha}: P_{\alpha} \rightarrow \omega_{2}$ for $\alpha<\omega_{2}$ such that the range of each $F_{\alpha}$ is an initial segment of $\omega_{2}$ which includes $\alpha$ and for $\beta<\alpha<\omega_{2}$, we have $F_{\beta} \subseteq F_{\alpha}$. (At each stage, $F_{\alpha}$ is any function satisfying these conditions.)

For $\alpha<\omega_{2}$, we make the following definitions after $P_{\alpha}$ and $F_{\alpha}$ are defined.

(6) $\dot{X}_{\alpha}$ denotes the $P_{\alpha}$-name for the set of real numbers whose elements have the names

$$
\bigcup_{n<\omega}\{n\} \times F_{\alpha}^{-1}\left[x_{\alpha}(\xi)(n)\right], \quad \xi<\alpha .
$$

(7) For each $i<\omega, \dot{A}_{\alpha i}$ and $\dot{B}_{\alpha i}$ denote the $\omega$-sequences of $P_{\alpha}$-names for real numbers

$$
\left\langle\bigcup_{n<\omega}\{n\} \times F_{\alpha}^{-1}\left[a_{\alpha}(i, j)(n)\right]: j<\omega\right\rangle
$$

and

$$
\left\langle\bigcup_{n<\omega}\{n\} \times F_{\alpha}^{-1}\left[b_{\alpha}(i, j)(n)\right]: j<\omega\right\rangle,
$$

respectively, and for each $\eta$ such that $\omega \leq \eta<\omega_{1}, \dot{A}_{\alpha \eta}$ and $\dot{B}_{\alpha \eta}$ denote the $\omega_{1}$-sequences of $P_{\alpha}$-names for real numbers

$$
\left\langle\bigcup_{n<\omega}\{n\} \times F_{\alpha}^{-1}\left[a_{\alpha}(\eta, \xi)(n)\right]: \xi<\omega_{1}\right\rangle
$$

and

$$
\left\langle\bigcup_{n<\omega}\{n\} \times F_{\alpha}^{-1}\left[b_{\alpha}(\eta, \xi)(n)\right]: \xi<\omega_{1}\right\rangle,
$$

respectively.

(8) $\dot{c}_{\alpha}$ denotes the $\omega$-sequence of $P_{\alpha}$-names for real numbers

$$
\left\langle\bigcup_{n<\omega}\{n\} \times F_{\alpha}^{-1}\left[c_{\alpha}(i)(n)\right]: i<\omega\right\rangle .
$$

(9) $\dot{f}_{\alpha}$ and $\dot{e}_{\alpha}$ denote respectively the $P_{\alpha}$-names for real numbers given by

$$
\bigcup_{n<\omega}\{n\} \times F_{\alpha}^{-1}\left[f_{\alpha}(n)\right] \text { and } \bigcup_{n<\omega}\{n\} \times F_{\alpha}^{-1}\left[e_{\alpha}(n)\right] .
$$

At stage $\alpha<\omega_{2}$ of the construction, if $\operatorname{cof}(\alpha)=\omega_{1}$ and if

$$
\Vdash_{P_{\alpha}} \dot{X}_{\alpha} \text { is second category, }
$$

then we use Lemma 1.14 to get a $P_{\alpha}$-name $\bar{M}_{\alpha}^{\prime}$ for an oracle so that if $P$ is any forcing notion which satisfies the $\bar{M}_{\alpha}^{\prime}$-cc, then $X_{\alpha}$ remains second category after forcing with $P$. Otherwise, in particular if $\operatorname{cof}(\alpha) \neq \omega_{1}$, we let $\bar{M}_{\alpha}^{\prime}$ be any $P_{\alpha}$-name for an oracle.

For $\beta<\alpha$, let $P_{\beta \alpha}$ be the usual $P_{\beta}$-name for a partial order such that $P_{\alpha}$ is isomorphic to a dense subset of $P_{\beta} * P_{\beta \alpha}$. Let $\bar{M}_{\beta \alpha}$ be a $P_{\alpha}$-name for an oracle such that

$$
\begin{gathered}
\text { If } \Vdash_{P_{\beta}} \text { " } P_{\beta, \alpha} \text { is } \bar{M}_{\beta} \text {-cc and } \Vdash_{P_{\beta, \alpha}} \dot{Q}_{\alpha} \text { is } \bar{M}_{\beta \alpha} \text {-cc", } \\
\text { then } \Vdash_{P_{\beta}} " P_{\beta, \alpha+1}=P_{\beta, \alpha} * \dot{Q}_{\alpha} \text { is } \bar{M}_{\beta} \text {-cc". }
\end{gathered}
$$

Let $\bar{M}_{\alpha}$ be a $P_{\alpha}$-name for an oracle such that

$$
\Vdash_{P_{\alpha}} \text { "If } \dot{Q}_{\alpha} \text { is } \bar{M}_{\alpha} \text {-cc, then } \dot{Q}_{\alpha} \text { is } \bar{M}_{\alpha}^{\prime} \text {-cc and } \bar{M}_{\beta \alpha} \text {-cc for all } \beta<\alpha \text { ". }
$$

Now, if $\operatorname{cof}(\alpha)=\omega_{1}$ and if

(12) $\Vdash_{P_{\alpha}}$ for $i<\omega$, the ranges of $\dot{A}_{\alpha i}, \dot{B}_{\alpha i}$ are dense in $\mathbb{R}$,

(13) $\Vdash_{P_{\alpha}}$ for $\eta$ such that $\omega \leq \eta<\omega_{1}$, the ranges of $\dot{A}_{\alpha \eta}, \dot{B}_{\alpha \eta}$ are everywhere second category, 
(14) $\Vdash_{P_{\alpha}} \dot{f}_{\alpha}$ is an entire function restricting to an order-isomorphism of $\mathbb{R}$ with a strictly positive derivative,

(15) $\Vdash_{P_{\alpha}} \dot{c}_{\alpha}(0) \leq \dot{c}_{\alpha}(1) \leq \ldots$ and $\lim _{i \rightarrow \infty} \dot{c}_{\alpha}(i)=\infty$, and

(16) $\Vdash_{P_{\alpha}} \dot{e}_{\alpha}$ is a positive continuous function,

then use Lemma 5.1 to get a $P_{\alpha}$-name $\dot{Q}_{\alpha}$ for a partial order satisfying the $\bar{M}_{\alpha}$-cc and forcing an entire function inducing an order-isomorphism between the $A_{\alpha \eta}$ and $B_{\alpha \eta}, \eta<\omega_{1}$, as described in the statement of the lemma. In all other cases, take $\dot{Q}_{\alpha}$ to name the partial order $Q$ for adding one Cohen real. We have thus

$$
\Vdash_{P_{\alpha}} \text { "Q } \dot{Q}_{\alpha} \text { satisfies the } \bar{M}_{\alpha} \text {-cc". }
$$

Now suppose that for some $P_{\omega_{2}}$-name $\dot{X}$ we have $\Vdash_{P_{\omega_{2}}} \dot{X}$ is second category. Fix a name $\dot{x}$ such that $\Vdash_{P_{\omega_{2}}} \dot{x}: \omega_{2} \rightarrow \dot{X}$ is onto. Then define $x: \omega_{2} \rightarrow\left(\left[\omega_{2}\right] \leq \omega\right) \omega$ so that if

$$
\tau_{\xi}=\bigcup_{n<\omega}\{n\} \times F^{-1}[x(\xi)(n)], \xi<\omega_{2},
$$

then for each $\xi<\omega_{2}, \Vdash_{P_{\omega_{2}}} \dot{x}(\xi)=\tau_{\xi}$. There is a closed unbounded set $C \subseteq \omega_{2}$ such that for each $\alpha \in C$ of cofinality $\omega_{1}$ we have:

(18) $x\left\lceil\alpha: \alpha \rightarrow([\alpha] \leq \omega)^{\omega}\right.$,

(19) $\forall \xi<\alpha, \tau_{\xi}$ is a $P_{\alpha}$-name,

(20) $\Vdash_{P_{\alpha}}\left\{\tau_{\xi}: \xi<\alpha\right\}$ is second category.

Choose such an $\alpha$ of cofinality $\omega_{1}$ for which $x\left\lceil\alpha=x_{\alpha}\right.$. By (19), the definition of $\tau_{\xi}$ for $\xi<\alpha$ would not change if we used $x_{\alpha}$ instead of $x$ and $F_{\alpha}$ instead of $F$. Then from the definition of $\dot{X}_{\alpha}$ we get

$$
\Vdash_{P_{\alpha}} \dot{X}_{\alpha}=\left\{\tau_{\xi}: \xi<\alpha\right\} .
$$

So at stage $\alpha$ we chose a $P_{\alpha}$-name $\bar{M}_{\alpha}$ and we arranged that $\Vdash_{P_{\alpha}}$ " $P_{\alpha, \gamma}$ is $\bar{M}_{\alpha}$-cc". (This follow by induction on $\gamma \geq \alpha$ using Proposition 1.15(1) at limits, and using (17), (11) and (10) above at successors.) Hence, by the choice of $\bar{M}_{\alpha}, \Vdash_{P_{\alpha}} \Vdash_{P_{\alpha, \gamma}}$ " $\dot{X}_{\alpha}$ is second category" from which it follows that $\Vdash_{P_{\alpha}} \Vdash_{P_{\alpha, \omega_{2}}}$ " $\dot{X}_{\alpha}$ is second category".

By what we have established, there are guaranteed to be sets of cardinality $\omega_{1}$ which are second category in any extension by $P_{\omega_{2}}$. Hence there are guaranteed to be everywhere second category sets of cardinality $\omega_{1}$. Suppose that for some $P_{\omega_{2}}$-names $\dot{A}_{\eta}, \dot{B}_{\eta}$ for $\eta<\omega_{1}, \dot{c}_{i}$ for $i<\omega$, and $\dot{f}, \dot{e}$ we have

(21) $\Vdash_{P_{\omega_{2}}}$ for $i<\omega$, the ranges of $\dot{A}_{i}, \dot{B}_{i}: \omega \rightarrow \mathbb{R}$ are dense in $\mathbb{R}$,

(22) $\Vdash_{P_{\omega_{2}}}$ for $\eta$ such that $\omega \leq \eta<\omega_{1}$, the ranges of $\dot{A}_{\eta}, \dot{B}_{\eta}: \omega_{1} \rightarrow \mathbb{R}$ are everywhere second category in $\mathbb{R}$,

(23) $\Vdash_{P_{\omega_{2}}} \dot{f}$ is an entire function restricting to an order-isomorphism of $\mathbb{R}$ with a strictly positive derivative,

(24) $\Vdash_{P_{\omega_{2}}} \dot{c}_{0} \leq \dot{c}_{1} \leq \ldots$ and $\lim _{i \rightarrow \infty} \dot{c}_{i}=\infty$, and

(25) $\Vdash_{P_{\omega_{2}}} \dot{e}$ is a positive continuous function.

Define $a, b, f, c, e$ to be functions as in (2)-(5) above so that letting

(26) $\sigma_{\eta \xi}=\bigcup_{n<\omega}\{n\} \times F^{-1}[a(\eta, \xi)(n)]$ and $\tau_{\eta \xi}=\bigcup_{n<\omega}\{n\} \times F^{-1}[b(\eta, \xi)(n)]$ for $\eta, \xi<\omega$ and for $\omega \leq \eta<\omega_{1}, \xi<\omega_{1}$, we have that for each such pair $\eta, \xi$, $\Vdash_{P_{\omega_{2}}} \dot{A}_{\eta}(\xi)=\sigma_{\eta \xi}$ and $\Vdash_{P_{\omega_{2}}} \dot{B}_{\eta}(\xi)=\tau_{\eta \xi}$;

(27) $\gamma_{i}=\bigcup_{n<\omega}\{n\} \times F^{-1}[c(i)(n)], i<\omega$, we have for each $i<\omega, \Vdash_{P_{\omega_{2}}} \dot{c}(i)=\gamma_{i}$; 
(28) $\varphi=\bigcup_{n<\omega}\{n\} \times F^{-1}[f(n)]$ and $\varepsilon=\bigcup_{n<\omega}\{n\} \times F^{-1}[e(n)]$, we have $\Vdash_{P_{\omega_{2}}}$ $\dot{f}=\varphi$ and $\Vdash_{P_{\omega_{2}}} \dot{e}=\varepsilon$.

For all large enough $\alpha<\omega_{2}$, we have

(29) $a, b: \omega_{1} \times \omega_{1} \rightarrow\left([\alpha]^{\leq \omega}\right)^{\omega}, f: \omega \rightarrow[\alpha]^{\leq \omega}, c: \omega \rightarrow\left([\alpha]^{\leq \omega}\right)^{\omega}$, and $e: \omega \rightarrow$ $[\alpha]^{\leq \omega}$

(30) for all values of the indices for which they are defined, $\sigma_{\eta \xi}, \tau_{\eta \xi}, \gamma_{i}, \varphi$ and $\varepsilon$ are $P_{\alpha}$-names.

Choose any such $\alpha$ of cofinality $\omega_{1}$ for which $(a, b, f, c, e)=\left(a_{\alpha}, b_{\alpha}, f_{\alpha}, c_{\alpha}, e_{\alpha}\right)$. By (30), the definitions of $\sigma_{\eta \xi}, \tau_{\eta \xi}, \gamma_{i}, \varphi$ and $\varepsilon$ would not change if we used $a_{\alpha}, b_{\alpha}, f_{\alpha}, c_{\alpha}, e_{\alpha}$ instead of $a, b, f, c, e$, respectively, and $F_{\alpha}$ instead of $F$. Then from the definitions of $\dot{A}_{\alpha i}, \dot{B}_{\alpha i}$ for $i<\omega, \dot{A}_{\alpha \eta}, \dot{B}_{\alpha \eta}$ for $\omega \leq \eta<\omega_{1}, \dot{f}_{\alpha}, \dot{c}_{\alpha}$, and $\dot{e}_{\alpha}$, we get that (12)-(16) hold. (Being everywhere second category is trivially downward absolute.) Then $\dot{Q}_{\alpha}$ was chosen to add an order isomorphism of the desired type and its properties are clearly upward absolute.

This completes the proof of the theorem.

\section{REFERENCES}

[ARS] U. Abraham, M. Rubin, S. Shelah, On the consistency of some partition theorems for continuous colorings, and the structure of $\aleph_{1}$-dense real order types, Ann. Pure Appl. Logic, 29 (1985) 123-206. MR801036 (87d:03132)

[Ba] J.E. Baumgartner, All $\aleph_{1}$-dense sets of reals can be isomorphic, Fund. Math., 79 (1973) 101-106. MR0317934 (47:6483)

[BS] K.F. Barth, W.J. Schneider, Entire functions mapping countable dense subsets of the reals onto each other monotonically, J. London Math. Soc. (2), 2 (1970) 620-626. MR0269834 (42:4729)

[BM] M.R. Burke, A.W. Miller, Models in which every nonmeager set is nonmeager in a nowhere dense Cantor set, Canad. J. Math., 57 (2005) 1139-1154. MR2178555 (2006g:03080)

[Ca] G. Cantor, Beiträge zur Begründung der transfiniten Mengenlehre, Math. Ann., 46 (1895) 481-512.

[Er] P. Erdős, Some unsolved problems, Michigan Math. J., 4 (1957) 291-300. MR0098702 (20:5157)

[Fr] P. Franklin, Analytic transformations of everywhere dense point sets, Trans. Amer. Math. Soc., 27 (1925) 91-100. MR.1501300

[Ho] L. Hoischen, Eine Verschärfung eines approximationssatzes von Carleman, J. Approximation Theory, 9 (1973) 272-277. MR0367217 (51:3459)

[Je] T. Jech, Set Theory, Academic Press, 1978. MR.506523 (80a:03062)

[Ku] K. Kunen, Set Theory, North-Holland, 1983. MR756630 (85e:03003)

[Ma] W.D. Maurer, Conformal equivalence of countable dense sets, Proc. Amer. Math. Soc., 18 (1967) 269-270. MR0215994 (35:6829)

[Me] Z.A. Melzak, Existence of certain analytic homeomorphisms, Canad. Math. Bull., 2 (1959) 71-75. MR0105474 (21:4215)

[NT] J.W. Nienhuys, J.G.F. Thiemann, On the existence of entire functions mapping countable dense sets onto each other, Nederl. Akad. Wetensch. Proc. Ser. A $79=$ Indag. Math., 38 (1976) 331-334. MR0460638 (57:631)

[Ru] W. Rudin, Real and Complex Analysis, 3rd ed., McGraw-Hill, New York, 1987. MR0924157 (88k:00002)

[SR] D. Sato, S. Rankin, Entire functions mapping countable dense subsets of the reals onto each other monotonically, Bull. Austral. Math. Soc., 10 (1974) 67-70. MR0346157 $(49: 10883)$

[Sh1980] S. Shelah, Independence results, J. Symbolic Logic, 45 (1980) 563-573. MR.583374 (82b:03099)

[Sh1998] - Proper and improper forcing, 2nd ed., Springer-Verlag, Berlin, 1998. MR:1623206 (98m:03002) 
[Wh] H. Whitney, Analytic extensions of differentiable functions defined in closed sets, Trans. Amer. Math. Soc., 36 (1934) 63-89. MR.1501735

[Zi] R.J. Zimmer, Essential results of functional analysis, University of Chicago Press, 1990. MR:1045444 (91h:46002)

Department of Mathematics and Statistics, University of Prince Edward Island, Charlottetown, Prince Edward Island, Canada C1A 4P3

E-mail address: burke@upei.ca 